Supporting Information for

\title{
Experimental and Theoretical Investigation of the Coarctate Cyclization of (2-Ethynylphenyl)phenyldiazenes
}

\author{
Laura D. Shirtcliff, Timothy J. R. Weakley, and Michael M. Haley* \\ Department of Chemistry, University of Oregon, Eugene, OR 97403-1253 \\ haley@oregon.uoregon.edu \\ Felix Köhler and Rainer Herges* \\ Institut für Organische Chemie, Universität Kiel, 24098 Kiel, Germany \\ rherges@oc.uni-kiel.de
}

$\underline{\text { Table of Contents }} \quad \underline{\text { Page }}$

$\begin{array}{lr}\text { Experimental procedures } & \mathrm{S} 2\end{array}$

$\begin{array}{lr}\text { Cartesian coordinates of calculated structures } & \text { S17 }\end{array}$

$\begin{array}{ll}\text { X-ray data for } \mathbf{1 1} & \text { S27 }\end{array}$

$\begin{array}{lr}\text { X-ray data for } \mathbf{1 2} & \mathrm{S} 40\end{array}$

$\begin{array}{lr}\text { NMR spectra } & \text { S55 }\end{array}$ 


\section{Experimental Section}

General. ${ }^{1} \mathrm{H}$ and ${ }^{13} \mathrm{C}$ NMR spectra were recorded on a $300 \mathrm{MHz}$ spectrometer $\left({ }^{1} \mathrm{H}, 299.95\right.$ $\mathrm{MHz} ;{ }^{13} \mathrm{C}, 75.43 \mathrm{MHz}$ ). Chemical shifts $(\delta)$ are expressed in ppm downfield from $\mathrm{SiMe}_{4}$ using the residual nondeuterated solvent as internal standard $\left(\mathrm{CDCl}_{3}:{ }^{1} \mathrm{H}, 7.26 \mathrm{ppm} ;{ }^{13} \mathrm{C}, 77.0 \mathrm{ppm}\right.$; $\left.\mathrm{C}_{4} \mathrm{D}_{8} \mathrm{O}:{ }^{13} \mathrm{C}, 67.57 \mathrm{ppm}\right)$. Coupling constants are expressed in hertz. IR spectra were recorded using a FTIR 550 spectrometer. Mass spectra were recorded using an LC mass spectrometer with either APCI or ESI sources. Melting points are uncorrected. $\mathrm{CH}_{2} \mathrm{Cl}_{2}$ and $\mathrm{Et}_{3} \mathrm{~N}$ were distilled from $\mathrm{CaH}_{2}$ under a nitrogen atmosphere prior to use. THF and $\mathrm{Et}_{2} \mathrm{O}$ were distilled from $\mathrm{Na} /$ benzophenone ketal under a $\mathrm{N}_{2}$ atmosphere prior to use. All other chemicals were of reagent grade quality and used as obtained from manufacturers. Reactions were carried out in an inert atmosphere (dry $\mathrm{N}_{2}$ or $\mathrm{Ar}$ ) when necessary. Column chromatography was performed on reagent grade silica gel (230-400 mesh). Preparative and analytical thin-layer chromatography was performed on precoated silica gel plates.

(2-Iodophenyl)phenyldiazene (9). 2-Iodoaniline (2.0 g, $9.13 \mathrm{mmol})$ and nitrosobenzene $(1.76 \mathrm{~g}, 16.4 \mathrm{mmol})$ were reacted according to general procedure B. The crude red oil was chromatographed over silica gel (hexanes) to yield $\mathbf{9}(1.88 \mathrm{~g}, 67 \%)$ as a red solid: $\mathrm{mp}$ 58.1-59.2 ${ }^{\circ} \mathrm{C} ;{ }^{1} \mathrm{H}$ NMR $\left(\mathrm{CDCl}_{3}\right) \delta$ 8.05-7.99 (m, 3H), $7.64(\mathrm{dd}, J=8.4,1.2 \mathrm{~Hz}, 1 \mathrm{H}), 7.58-7.50(\mathrm{~m}, 3 \mathrm{H})$, $7.44(\mathrm{dt}, J=8.4,1.2 \mathrm{~Hz}, 1 \mathrm{H}), 7.17(\mathrm{dt}, J=8.4,1.2 \mathrm{~Hz}, 1 \mathrm{H}) ;{ }^{13} \mathrm{C} \mathrm{NMR}\left(\mathrm{CDCl}_{3}\right) \delta 152.3,151.2$, 139.8, 132.2, 131.6, 129.2, 128.9, 123.5, 117.3, 102.5; IR (KBr) 3461, 3361, 2962, 2905, 1585 $\mathrm{cm}^{-1}$; MS (ESI) $m / z(\%) 309.0\left(100, \mathrm{M}^{+}+\mathrm{H}\right), 182.2\left(20, \mathrm{M}^{+}-\mathrm{I}^{127}\right)$.

Phenyl[2-(trimethylsilylethynyl)phenyl]diazene (6). Iododiazene 9 (0.68 g, $2.20 \mathrm{mmol})$, TMSA (0.44 mL, $3.08 \mathrm{mmol}), \mathrm{PdCl}_{2}$ (dppf) (0.09 g, $\left.0.11 \mathrm{mmol}\right), \mathrm{CuI}(41 \mathrm{mg}, 0.22 \mathrm{mmol})$, and THF/DIPA (1:1) were reacted according to general procedure D. Chromatography on silica gel (hexanes) afforded $6(0.305 \mathrm{~g}, 50 \%)$ as a viscous red oil: ${ }^{1} \mathrm{H} \mathrm{NMR}\left(\mathrm{CDCl}_{3}\right) \delta$ 8.01-7.98 (m, 2H), 7.72-7.63 (m, 2H), 7.56-7.47 (m, 3H), 7.44-7.37 (m, 2H) 0.23 (s, 9H); ${ }^{13} \mathrm{C} \mathrm{NMR}\left(\mathrm{CDCl}_{3}\right) \delta$ $152.8,152.5,133.6,131.3,130.5,129.1,129.0,122.8,123.3,115.8,101.9,101.2,-0.1 ; \mathrm{IR}(\mathrm{KBr})$ $3057,2959,2159,1473 \mathrm{~cm}^{-1}$; MS (ESI) $m / z(\%) 279.1\left(100, \mathrm{M}^{+}+\mathrm{H}\right)$. 
Phenyl[2-(triisopropylsilylethynyl)phenyl]diazene (7). Iododiazene 9 (1.77 g, $5.74 \mathrm{mmol})$, TIPSA (1.80 mL, $8.03 \mathrm{mmol}), \mathrm{PdCl}_{2}\left(\mathrm{PPh}_{3}\right)_{2}(0.08 \mathrm{~g}, 0.114 \mathrm{mmol}), \mathrm{CuI}(0.109 \mathrm{~g}, 0.574 \mathrm{mmol})$, and $\mathrm{Et}_{3} \mathrm{~N}$ were reacted according to general procedure D. Chromatography on silica gel (0-10\% $\mathrm{CH}_{2} \mathrm{Cl}_{2}$ in hexanes gradient) afforded $7(2.02 \mathrm{~g}, 97 \%)$ as a viscous red oil: ${ }^{1} \mathrm{H} \mathrm{NMR}\left(\mathrm{CDCl}_{3}\right) \delta$ 8.02-7.98 (m, 2H), 7.71-7.66 (m, 2H), 7.53-7.45 (m, 3H), 7.24-7.38 (m, 2H), $1.16(\mathrm{~s}, 21 \mathrm{H}) ;{ }^{13} \mathrm{C}$ $\operatorname{NMR}\left(\mathrm{CDCl}_{3}\right) \delta 153.5,152.8,134.1,131.2,130.3,129.1,128.9,124.0,123.4,115.7,103.8$, 97.5, 18.7, 11.4; IR (neat) 2942, 2864, 2155, $1480 \mathrm{~cm}^{-1}$; MS (ESI) $\mathrm{m} / z$ (\%) $363.2\left(80, \mathrm{M}^{+}+\mathrm{H}\right.$ ), $385.2\left(100, \mathrm{M}^{+}+\mathrm{Na}\right), 206.3\left(20, \mathrm{M}^{+}-\mathrm{C}_{9} \mathrm{H}_{21} \mathrm{Si}\right)$.

Phenyl[2-(triethylsilylethynyl)phenyl]diazene (8). Iododiazene 9 (1.45 g, $4.71 \mathrm{mmol}$ ), TESA (1.18 mL, $6.60 \mathrm{mmol}), \mathrm{PdCl}_{2}\left(\mathrm{PPh}_{3}\right)_{2}(0.07 \mathrm{~g}, 0.094 \mathrm{mmol}), \mathrm{CuI}(0.09 \mathrm{~g}, 0.471 \mathrm{mmol})$, and $\mathrm{Et}_{3} \mathrm{~N}$ were reacted according to general procedure D. Chromatography on silica gel (packed with hexanes and eluted with $5 \% \mathrm{CH}_{2} \mathrm{Cl}_{2}$ in hexanes) furnished $\mathbf{8}(1.31 \mathrm{~g}, 87 \%)$ as a viscous red oil: ${ }^{1} \mathrm{H}$ NMR $\left(\mathrm{CDCl}_{3}\right)$ d 8.02-7.98 (m, 2H), 7.71-7.65 (m, 2H), 7.50-7.47 (m, 3H), 7.41-7.38 (m, 2H) $1.07(\mathrm{t}, J=7.8 \mathrm{~Hz}, 9 \mathrm{H}) 0.72(\mathrm{q}, J=7.8,6 \mathrm{H}) ;{ }^{13} \mathrm{C} \mathrm{NMR}\left(\mathrm{CDCl}_{3}\right) \delta 153.5,152.8,133.9,131.3$, 130.4, 130.0, 128.9, 123.7, 123.4, 115.7, 103.0, 98.7, 7.5, 4.4; IR (neat) 2954, 2910, 2874, 2156, $1474 \mathrm{~cm}^{-1}$; MS (ESI) $m / z(\%) 321.2\left(100, \mathrm{M}^{+}+\mathrm{H}\right), 293.2\left(30, \mathrm{M}^{+}-\mathrm{C}_{2} \mathrm{H}_{3}\right), 279.2\left(40, \mathrm{M}^{+}-\mathrm{C}_{3} \mathrm{H}_{5}\right)$.

2-Phenyl-2H-indazole-3-carbaldehyde (10). Diazene 7 (404 mg, $1.11 \mathrm{mmol}$ ) was desilylated with TBAF $(5.55 \mathrm{~mL}, 5.55 \mathrm{mmol})$ according to the first step of general procedure E. After concentrating in vacuo with no heat, the red oil was immediately taken up in DCE (150 $\mathrm{mL})$ and cooled to $-5{ }^{\circ} \mathrm{C}$. $\mathrm{CuCl}(219 \mathrm{mg}, 2.22 \mathrm{mmol})$ was added and the reaction allowed to stir until all s.m. was consumed (by TLC). The reaction mixture was filtered over a short pad of silica and concentrated in vacuo. Chromatography on silica gel $\left(10 \% \mathrm{CH}_{2} \mathrm{Cl}_{2}, 10 \%\right.$ EtOAc, $80 \%$ hexanes) afforded $\mathbf{1 0}(25 \mathrm{mg}, 10 \%)$ as a tan solid: $\mathrm{mp} 73.1-74.6{ }^{\circ} \mathrm{C} ;{ }^{1} \mathrm{H} \mathrm{NMR}\left(\mathrm{CDCl}_{3}\right) \delta 10.12$ (s, 1H), $8.32(\mathrm{dt}, J=9.0,0.9 \mathrm{~Hz}, 1 \mathrm{H}), 7.91(\mathrm{dt}, J=9.0,0.9 \mathrm{~Hz}, 1 \mathrm{H}), 7.70-7.60(\mathrm{~m}, 5 \mathrm{H}), 7.52-$ $7.42(\mathrm{~m}, 2 \mathrm{H}) ;{ }^{13} \mathrm{C} \mathrm{NMR}\left(\mathrm{CDCl}_{3}\right) \delta$ 180.0, 148.5, 130.1, 129.6, 129.5, 129.2, 127.8, 127.1, 126.4, 121.1, 120.9, 118.9; IR (KBr) 3055, 2858, 1667, $1596 \mathrm{~cm}^{-1}$; MS (APCI) $\mathrm{m} / z$ (\%) $223.2(100$, $\mathrm{M}^{+}+\mathrm{H}$ ). Isolation of the second band and subsequent recrystallization from $\mathrm{CHCl}_{3}$ furnished $\mathbf{1 1}$ 
(25 mg, 11\%) as yellow needles suitable for X-ray diffraction: $\mathrm{mp} 136.5-138.0{ }^{\circ} \mathrm{C} ;{ }^{1} \mathrm{H} \mathrm{NMR}$ $\left(\mathrm{CDCl}_{3}\right) \delta 7.93-7.91(\mathrm{~m}, 4 \mathrm{H}), 7.85(\mathrm{~d}, J=9.0 \mathrm{~Hz}, 2 \mathrm{H}), 7.71(\mathrm{~d}, J=8.4 \mathrm{~Hz}, 2 \mathrm{H}) 7.61-7.48(\mathrm{~m}$, $6 \mathrm{H}), 7.41(\mathrm{td}, J=7.5,1.2 \mathrm{~Hz}, 2 \mathrm{H}), 7.27(\mathrm{td}, J=7.5,1.2 \mathrm{~Hz}, 2 \mathrm{H}) ;{ }^{13} \mathrm{C} \mathrm{NMR}\left(\mathrm{CDCl}_{3}\right) \delta 148.8$, 129.6, 129.1, 129.0, 127.5, 126.5, 124.5, 123.9, 119.8, 118.6, 117.4, 89.0; IR (KBr) 3061, 2921, 1959, 1886, $1502 \mathrm{~cm}^{-1}$; MS (ESI) $m / z(\%) 411.1\left(100, \mathrm{M}^{+}+\mathrm{H}\right), 333.1\left(20, \mathrm{M}^{+}-\mathrm{C}_{6} \mathrm{H}_{5}\right), 305.2$ (40, $\left.\mathrm{M}^{+}-\mathrm{C}_{6} \mathrm{H}_{5} \mathrm{~N}_{2}\right) 217.0\left(15, \mathrm{M}^{+}-\mathrm{C}_{13} \mathrm{H}_{9} \mathrm{~N}_{2}\right)$. Isolation of the third band afforded $12(40 \mathrm{mg}, 9 \%)$ as a light clear violet oil: ${ }^{1} \mathrm{H}$ NMR $\left(\mathrm{CDCl}_{3}\right) \delta$ 7.84-7.75 (m, 4H), 7.56-7.48 (m, 3H) 7.34 (td, $J=9.0$, $0.9 \mathrm{~Hz}, 1 \mathrm{H}) 7.14(\mathrm{td}, J=9.0,0.9 \mathrm{~Hz}, 1 \mathrm{H}), 5.07(\mathrm{~s}, 2 \mathrm{H}), 1.30(\mathrm{~s}, 21 \mathrm{H}) ;{ }^{13} \mathrm{C} \mathrm{NMR}\left(\mathrm{CDCl}_{3}\right) \delta$ $148.5,139.8,129.0,128.7,126,6,125.6,121.9,121.8,119.9,117.8,55.0,18.0,11.9$; IR (KBr) 3057, 2941, 2863, 1605, $1460 \mathrm{~cm}^{-1}$; MS (ESI) $\mathrm{m} / z(\%) 381.1\left(80, \mathrm{M}^{+}+\mathrm{H}\right), 207.2$ $\left(\mathrm{M}^{+}-\mathrm{SiC}_{9} \mathrm{H}_{21} \mathrm{O}\right)$.

Alkyne 11 by Mori's method. ${ }^{20}$ To diazene 6 (141 mg, $\left.0.50 \mathrm{mmol}\right)$ in DMSO $(7 \mathrm{~mL})$ was added $\mathrm{CuCl}(90 \mathrm{mg}, 2.0 \mathrm{mmol})$. After $30 \mathrm{~min}$ the reaction was diluted with $\mathrm{Et}_{2} \mathrm{O}$ and the organic layer washed successively with $30 \%$ aq. $\mathrm{NH}_{4} \mathrm{OH}(2 \times)$, brine $(2 \times)$, and $\mathrm{H}_{2} \mathrm{O}(2 \mathrm{x})$, dried $\left(\mathrm{MgSO}_{4}\right)$, filtered over a short pad of silica $\left(\mathrm{Et}_{2} \mathrm{O}\right)$ and concentrated. The brown solid was dissolved in a minimal amount of $\mathrm{Et}_{2} \mathrm{O}$ and the product was precipitated with hexanes to yield 11 (45 mg, 45\%) as a fluffy yellow solid. Spectral data were consistent with those given above.

2-Phenyl-3-triethylsilyloxymethyl-2H-indazole (13). Diazene 8 (336 mg, $1.0 \mathrm{mmol}$ ) was desilylated with TBAF $(2.0 \mathrm{~mL}, 2.0 \mathrm{mmol})$ according to the first step of general procedure E. After concentrating in vacuo, the red oil was dissolved in DCE $(20 \mathrm{~mL})$ and immediately degassed by four successive freeze/pump/thaw cycles. TESOH (357 mg, $2.7 \mathrm{mmol}$ ) was added and the reaction was stirred in a preheated $75^{\circ} \mathrm{C}$ sand bath for $17 \mathrm{~h}$. The brown solution was filtered over a short pad of silica and concentrated in vacuo. Chromatography on silica gel (7\% $\mathrm{CH}_{2} \mathrm{Cl}_{2}, 7 \%$ EtOAc, 86\% hexanes) afforded 13 (262 mg, 77\%) as a purplish oil: ${ }^{1} \mathrm{H}$ NMR $\left(\mathrm{CDCl}_{3}\right) \delta$ 7.82-7.73 (m, 4H), 7.56-7.48 (m, 3H), 7.36-7.31 (m, 1H) 7.17-7.12 (m, 1H), $4.98(\mathrm{~s}$ 2H), $0.96(\mathrm{t}, J=8.1 \mathrm{~Hz}, 9 \mathrm{H}), 0.66(\mathrm{q}, J=8.1 \mathrm{~Hz}, 6 \mathrm{H}) ;{ }^{13} \mathrm{C} \mathrm{NMR}\left(\mathrm{CDCl}_{3}\right) \delta$ 148.6, 139.8, 133.9, $129.1,128.7,126.7,125.5,122.0,121.8,119.7,117.8,54.4,6.7,4.3$. 
(2-Iodo-4-methylphenyl)phenyldiazene (18). $p$-Toluidine (1.50 g, $14.0 \mathrm{mmol}), \mathrm{BTEA} \mathrm{ICl}_{2}$ $(6.55 \mathrm{~g}, 16.8 \mathrm{mmol})$, and $\mathrm{CaCO}_{3}(1.96 \mathrm{~g}, 19.60 \mathrm{mmol})$ were reacted using general procedure A. The iodoaniline $(3.07 \mathrm{~g}, 95 \%)$ was obtained as a dark purple oil and used without further purification. A portion of the crude product $(1.50 \mathrm{~g}, 6.46 \mathrm{mmol})$ and nitrosobenzene $(1.24 \mathrm{~g}$, $11.63 \mathrm{mmol}$ ) were reacted according to general procedure B. Chromatography on silica gel (hexanes) afforded $18(1.46 \mathrm{~g}, 70 \%)$ as a red solid: $\mathrm{mp} 58.5-60.1{ }^{\circ} \mathrm{C} ;{ }^{1} \mathrm{H} \mathrm{NMR}\left(\mathrm{CDCl}_{3}\right) \delta 8.02-$ $8.00(\mathrm{~m}, 2 \mathrm{H}), 7.99(\mathrm{~d}, J=1.5 \mathrm{~Hz}, 1 \mathrm{H}), 7.58-7.48(\mathrm{~m}, 4 \mathrm{H}), 7.23(\mathrm{dd}, J=6.6,0.6 \mathrm{~Hz}, 1 \mathrm{H}), 2.41(\mathrm{~s}$, $3 \mathrm{H}) ;{ }^{13} \mathrm{C} \mathrm{NMR}\left(\mathrm{CDCl}_{3}, 75 \mathrm{MHz}\right) \delta 152.3,149.2,143.1,140.1,131.3,129.7,129.1,123.4,116.8$, 103.3, 20.9; IR (KBr) 3046, 2907, 1585, $1473 \mathrm{~cm}^{-1}$; MS (ESI) $m / z(\%) 323.0\left(25, \mathrm{M}^{+}+\mathrm{H}\right), 245.0$ $\left(30, \mathrm{M}^{+}-\mathrm{C}_{6} \mathrm{H}_{5}\right), 217.0\left(40, \mathrm{M}^{+}-\mathrm{C}_{6} \mathrm{H}_{5} \mathrm{~N}_{2}\right), 196.0\left(20, \mathrm{M}^{+}-\mathrm{I}^{125}\right), 194.0\left(100, \mathrm{M}^{+}-\mathrm{I}^{127}\right)$.

(4-tert-Butyl-2-iodophenyl)phenyldiazene (19). 4-tert-Butylaniline (0.796 mL, $5 \mathrm{mmol})$, $\mathrm{BTEA} \cdot \mathrm{ICl}_{2}(2.18 \mathrm{~g}, 5.6 \mathrm{mmol})$, and $\mathrm{CaCO}_{3}(0.7 \mathrm{~g}, 7 \mathrm{mmol})$ were reacted using general procedure A. The iodoaniline (1.35 g, 98\%) was obtained as a dark purple solid and used without further purification. A portion of the crude product $(0.60 \mathrm{~g}, 2.2 \mathrm{mmol})$ and nitrosobenzene $(0.471 \mathrm{~g}, 4.4 \mathrm{mmol})$ were reacted according to general procedure B. Chromatography on silica gel (hexanes) gave $19(0.583 \mathrm{~g}, 72 \%)$ as a viscous, bright red oil: ${ }^{1} \mathrm{H}$ $\operatorname{NMR}\left(\mathrm{CDCl}_{3}\right) \delta 8.03-8.01(\mathrm{~m}, 3 \mathrm{H}), 7.62-7.44(\mathrm{~m}, 5 \mathrm{H}), 1.36(\mathrm{~s}, 9 \mathrm{H}) ;{ }^{13} \mathrm{C} \mathrm{NMR}\left(\mathrm{CDCl}_{3}\right) \delta 156.2$, 152.4, 149.1 136.8, 131.3, 129.1, 126.2, 123.4, 116.7, 103.3, 34.9, 31.1; IR (neat) 3459, 2963, 2906, 1585, $1548 \mathrm{~cm}^{-1}$; MS (ESI) $m / z(\%) 365.1\left(100, \mathrm{M}^{+}+\mathrm{H}\right), 350.0\left(80, \mathrm{M}^{+}-\mathrm{CH}_{3}\right) 335.0(17$, $\left.\mathrm{M}^{+}-2 \mathrm{CH}_{3}\right)$.

(4-Chloro-2-iodophenyl)phenyldiazene (20). 4-Chloroaniline (0.87 g, 6.84 mmol), $\mathrm{BTEA} \cdot \mathrm{ICl}_{2}(3.20 \mathrm{~g}, 8.21 \mathrm{mmol})$, and $\mathrm{CaCO}_{3}(0.96 \mathrm{~g}, 9.58 \mathrm{mmol})$ were reacted using general procedure A. The iodoaniline $(1.40 \mathrm{~g}, 80 \%)$ was obtained as a dark purple oil and used without further purification. A portion of the crude product $(1.0 \mathrm{~g}, 3.94 \mathrm{mmol})$ and nitrosobenzene $(0.76$ g, $7.09 \mathrm{mmol}$ ) were reacted according to general procedure B. Chromatography on silica gel (5\% $\mathrm{CH}_{2} \mathrm{Cl}_{2} /$ hexanes) furnished $20(0.547 \mathrm{~g}, 41 \%)$ as a red solid: $\mathrm{mp} 108.5-109.6{ }^{\circ} \mathrm{C} ;{ }^{1} \mathrm{H} \mathrm{NMR}$ $\left(\mathrm{CDCl}_{3}\right) \delta 8.03(\mathrm{~d}, J=1.8 \mathrm{~Hz}, 1 \mathrm{H}), 8.00-7.97(\mathrm{~m}, 2 \mathrm{H}), 7.60(\mathrm{~d}, J=8.4 \mathrm{~Hz}, 1 \mathrm{H}), 7.56-7.51(\mathrm{~m}$, 
3H), $7.41(\mathrm{dd}, J=8.4,1.8 \mathrm{~Hz}, 1 \mathrm{H}) ;{ }^{13} \mathrm{C} \mathrm{NMR}\left(\mathrm{CDCl}_{3}\right) \delta 152.12,149.8,149.1,137.4,131.8$, 129.2, 123.6, 120.4, 117.8, 103.3; IR (KBr) 3056, 2920, 1565, $1449 \mathrm{~cm}^{-1}$; MS (ESI) $\mathrm{m} / z$ (\%) $365.0\left(25, \mathrm{M}^{+}+\mathrm{Na}\right), 343.0\left(100, \mathrm{M}^{+}+\mathrm{H}\right), 267.0\left(15, \mathrm{M}^{+}-\mathrm{C}_{6} \mathrm{H}_{3}\right), 265.0\left(55, \mathrm{M}^{+}-\mathrm{C}_{6} \mathrm{H}_{5}\right) 237.0(40$, $\mathrm{M}^{+}-\mathrm{C}_{6} \mathrm{H}_{5} \mathrm{~N}_{2}$ ).

(2,4-Diiodophenyl)phenyldiazene (21). 4-Iodoaniline (1.50 g, $6.84 \mathrm{mmol}), \mathrm{BTEA} \cdot \mathrm{ICl}_{2}$ $(3.20 \mathrm{~g}, 8.21 \mathrm{mmol})$, and $\mathrm{CaCO}_{3}(0.96 \mathrm{~g}, 9.59 \mathrm{mmol})$ were reacted using general procedure $\mathrm{A}$. The iodoaniline (2.27 g, 96\%) was obtained as a dark purple solid and used without further purification. The crude product $(2.26 \mathrm{~g}, 6.58 \mathrm{mmol})$ and nitrosobenzene $(1.27 \mathrm{~g}, 11.8 \mathrm{mmol})$ were reacted according to general procedure B. Chromatography on silica gel (hexanes) afforded $21(0.44 \mathrm{~g}, 32 \%)$ as a red solid: $\mathrm{mp} 115.8-116.8{ }^{\circ} \mathrm{C} ;{ }^{1} \mathrm{H}$ NMR $\left(\mathrm{CDCl}_{3}\right) \delta 8.39(\mathrm{~d}, J=1.8$ $\mathrm{Hz}, 1 \mathrm{H}), 8.00-7.97(\mathrm{~m}, 2 \mathrm{H}), 7.50(\mathrm{dd}, J=9.0,1.8 \mathrm{~Hz}, 1 \mathrm{H}), 7.56-7.51(\mathrm{~m}, 3 \mathrm{H}), 7.37(\mathrm{~d}, J=9.0$ $\mathrm{Hz}, 1 \mathrm{H}) ;{ }^{13} \mathrm{C} \mathrm{NMR}\left(\mathrm{CDCl}_{3}\right) \delta 152.1,150.8,147.5,138.1,131.9,129.7,123.7,118.4,103.4,98.1$; IR (KBr) 3060, 2908, 1555, $1445 \mathrm{~cm}^{-1}$; MS (ESI) $\mathrm{m} / z$ (\%) $435.0\left(40, \mathrm{M}^{+}+\mathrm{H}\right), 357.0$ (100, $\left.\mathrm{M}^{+}-\mathrm{C}_{6} \mathrm{H}_{5}\right), 307.0\left(25, \mathrm{M}^{+}-\mathrm{I}^{127}\right), 201.9\left(100, \mathrm{M}^{+}-\mathrm{C}_{6} \mathrm{H}_{5} \mathrm{~N}_{2} \mathrm{I}\right)$.

[4-Methyl-2-(triethylsilylethynyl)phenyl]phenyldiazene (14). Iododiazene 18 (1.0 g, 3.10 mmol), TESA (0.79 mL, $4.34 \mathrm{mmol}), \mathrm{PdCl}_{2}\left(\mathrm{PPh}_{3}\right)_{2}(0.043 \mathrm{~g}, 0.062 \mathrm{mmol}), \mathrm{CuI}(0.06 \mathrm{~g}, 0.310$ $\mathrm{mmol})$, and $\mathrm{Et}_{3} \mathrm{~N}(31 \mathrm{~mL})$ were reacted according to general procedure D. Chromatography on silica gel (hexanes) gave 14 (1.02 g, 98\%) as a red viscous oil: ${ }^{1} \mathrm{H}$ NMR $\left(\mathrm{CDCl}_{3}\right) \delta$ 8.01-7.98 (m, 2H), 7.65 (d, $J=8.5 \mathrm{~Hz}, 1 \mathrm{H}), 7.52-7.48(\mathrm{~m}, 4 \mathrm{H}), 7.21$ (dd, $J=8.5,1.5 \mathrm{~Hz}, 1 \mathrm{H}), 2.14(\mathrm{~s}, 3 \mathrm{H})$, 1.08 (q, $J=7.5 \mathrm{~Hz}, 9 \mathrm{H}), 0.74(\mathrm{t}, J=7.5 \mathrm{~Hz}, 6 \mathrm{H}) ;{ }^{13} \mathrm{C} \mathrm{NMR}\left(\mathrm{CDCl}_{3}\right) \delta 152.9,151.5,141.0$, 134.2, 131.0, 130.0, 128.9, 123.9, 123.3, 115.5, 103.3, 100.0, 21.2, 7.6, 4.5; IR (neat) 2954, 2911, 2874, 2151, $1598 \mathrm{~cm}^{-1}$; MS (APCI) $m / z$ (\%) $335.2\left(100, \mathrm{M}^{+}+\mathrm{H}\right)$.

[4-tert-Butyl-2-(triethylsilylethynyl)phenyl]phenyldiazene (15). Iododiazene 19 (1.43 g, $3.93 \mathrm{mmol})$, TESA (0.99 mL, $5.50 \mathrm{mmol}), \mathrm{PdCl}_{2}\left(\mathrm{PPh}_{3}\right)_{2}(0.055 \mathrm{~g}, 0.079 \mathrm{mmol}), \mathrm{CuI}(0.075 \mathrm{~g}$, $0.393 \mathrm{mmol})$, and $\mathrm{Et}_{3} \mathrm{~N}(40 \mathrm{~mL})$ were reacted according to general procedure $\mathrm{C}$.

Chromatography on silica gel (hexanes) furnished 15 (1.48 g, 78\%) as a red viscous oil: ${ }^{1} \mathrm{H}$ NMR $\left(\mathrm{CDCl}_{3}\right) \delta$ 8.00-7.98 (m, 2H), 7.66-7.63 (m, 2H), 7.51-7.42 (m, 4H), 1.37 (s, 9H), 1.08 (t, $J=7.8$ 
$\mathrm{Hz}, 9 \mathrm{H}), 0.75$ (q, $J=7.8 \mathrm{~Hz}, 6 \mathrm{H}) ;{ }^{13} \mathrm{C} \mathrm{NMR}\left(\mathrm{CDCl}_{3}\right) \delta 153.9,152.9,151.4,131.0,130.5,128.9$, 126.5, 123.5, 123.3, 115.3, 103.7, 97.8, 34.9, 31.1, 7.6, 4.5; IR (neat) 2956, 2910, 2874, 2155, $1593 \mathrm{~cm}^{-1}$; MS (APCI) $m / z(\%) 377.2\left(100, \mathrm{M}^{+}+\mathrm{H}\right), 319.1\left(25, \mathrm{M}^{+}-\mathrm{C}_{4} \mathrm{H}_{9}\right)$.

[4-Chloro-2-(triethylsilylethynyl)phenyl]phenyldiazene (16). Iododiazene 20 (0.40 g, $1.17 \mathrm{mmol})$, TESA (0.29 mL, $1.63 \mathrm{mmol}), \mathrm{PdCl}_{2}\left(\mathrm{PPh}_{3}\right)_{2}(0.016 \mathrm{~g}, 0.023 \mathrm{mmol}), \mathrm{CuI}(0.022 \mathrm{~g}$, $0.117 \mathrm{mmol})$, and $\mathrm{Et}_{3} \mathrm{~N}(12 \mathrm{~mL})$ were reacted according to general procedure $\mathrm{D}$.

Chromatography on silica gel $\left(9: 1\right.$ hexanes $\left./ \mathrm{CH}_{2} \mathrm{Cl}_{2}\right)$ gave $\mathbf{1 6}(1.02 \mathrm{~g}, 97 \%)$ as a red viscous oil: ${ }^{1} \mathrm{H}$ NMR $\left(\mathrm{CDCl}_{3}\right) \delta$ 8.00-7.97 (m, 2H), 7.67-7.64 (m, 2H), 7.52-7.48 (m, 3H), 7.35 (dd, $J=8.7$, $2.4 \mathrm{~Hz}, 1 \mathrm{H}) ;{ }^{13} \mathrm{C} \mathrm{NMR}\left(\mathrm{CDCl}_{3}\right) \delta$ 152.7, 151.8, 136.3, 133.4, 131.5, 129.3, 129.00, 125.3, 123.4, 117.0, 101.6, 100.3, 7.3, 4.4; IR (KBr) 3065, 2955, 2934, 2158, $1583 \mathrm{~cm}^{-1}$; MS (APCI) $\mathrm{m} / z(\%)$ $355.1\left(100, \mathrm{M}^{+}+\mathrm{H}\right)$.

[2,4-Bis(triethylsilylethynyl)phenyl]phenyldiazene (17). Iododiazene 21 ( $0.35 \mathrm{~g}, 0.81$ mmol), TESA (0.36 mL, $2.01 \mathrm{mmol}), \mathrm{PdCl}_{2}\left(\mathrm{PPh}_{3}\right)_{2}(0.03 \mathrm{~g}, 0.04 \mathrm{mmol}), \mathrm{CuI}(0.023 \mathrm{~g}, 0.121$ $\mathrm{mmol})$ and $\mathrm{Et}_{3} \mathrm{~N}(10 \mathrm{~mL})$ were reacted according to general procedure D. Chromatography on silica gel $\left(0-5 \% \mathrm{CH}_{2} \mathrm{Cl}_{2}\right.$ gradient in hexanes) furnished $17(0.314 \mathrm{~g}, 85 \%)$ as a red viscous oil: ${ }^{1} \mathrm{H}$ NMR $\left(\mathrm{CDCl}_{3}\right) \delta$ 8.00-7.97 (m, 2H), $7.76(\mathrm{~d}, J=1.8 \mathrm{~Hz}, 1 \mathrm{H}), 7.65(\mathrm{~d}, J=8.4 \mathrm{~Hz}, 1 \mathrm{H}), 7.51-7.45$ (m, 4H), $1.06(\mathrm{~m}, 18 \mathrm{H}), 0.70(\mathrm{~m}, 12 \mathrm{H}) ;{ }^{13} \mathrm{C} \mathrm{NMR}\left(\mathrm{CDCl}_{3}\right) \delta$ 152.8, 152.3, 137.3, 132.5, 131.5, 128.3, 125.7, 123.5, 120.5, 115.7, 105.0, 102.2, 99.4, 95.2, 7.5, 7.5, 4.4, 4.3; IR (neat) 2955, 2911, 2874, 2150, 2064, 1594; MS (APCI) $m / z(\%) 459.1\left(100, \mathrm{M}^{+}+\mathrm{H}\right)$.

(2-Iodo-4-methoxyphenyl)phenyldiazene (24). Aniline (1.0 g, $10.74 \mathrm{mmol})$, conc. $\mathrm{HCl}$ (3.57 mL, $21.47 \mathrm{mmol}), \mathrm{NaNO}_{2}(0.89 \mathrm{~g}, 12.88 \mathrm{mmol}), \mathrm{KOH}(2.20 \mathrm{~g}, 21.47 \mathrm{mmol})$, and 3iodophenol (2.60 g, $11.81 \mathrm{mmol})$ were reacted according to general procedure $\mathrm{C}$, part 1 . The resultant red solid $(3.45 \mathrm{~g})$ was reacted with $\mathrm{MeI}(1.21 \mathrm{~mL}, 19.44 \mathrm{mmol})$ and $\mathrm{Cs}_{2} \mathrm{CO}_{3}(7.25 \mathrm{~g}$, $22.3 \mathrm{mmol})$ in DMF $(22 \mathrm{~mL})$ according to general procedure $\mathrm{C}$, part 2. Recrystallization from EtOH/ $\mathrm{H}_{2} \mathrm{O}$ yielded $24(2.75 \mathrm{~g}, 77 \%)$ as a red solid: $\mathrm{mp} 44.8-46.6{ }^{\circ} \mathrm{C} ;{ }^{1} \mathrm{H} \mathrm{NMR}\left(\mathrm{CDCl}_{3}\right) \delta 7.98$ $7.95(\mathrm{~m}, 2 \mathrm{H}), 7.69(\mathrm{~d}, J=8.8 \mathrm{~Hz}, 1 \mathrm{H}), 7.55-7.46(\mathrm{~m}, 4 \mathrm{H}), 6.97(\mathrm{dd}, J=8.8,2.3 \mathrm{~Hz}, 1 \mathrm{H}), 3.88(\mathrm{~s}$, $3 \mathrm{H}) ;{ }^{13} \mathrm{C} \mathrm{NMR}\left(\mathrm{CDCl}_{3}\right) \delta 162.1,152.3,130.9,129.1,128.8,123.8,123.3,122.3,115.4,105.2$, 
55.8; IR (KBr) 3065, 2963, 2834, 1587, $1480 \mathrm{~cm}^{-1}$; MS (ESI) $m / z$ (\%) $339.0\left(100, \mathrm{M}^{+}+\mathrm{H}\right), 261.0$ $\left(30, \mathrm{M}^{+}-\mathrm{C}_{6} \mathrm{H}_{5}\right), 212.0\left(15, \mathrm{M}^{+}-\mathrm{I}^{125}\right), 210.9\left(55, \mathrm{M}^{+}-\mathrm{I}^{127}\right)$.

(4-Bromophenyl)(2-iodo-4-methoxyphenyl)diazene (25). 4-Bromoaniline (1.25 g, 7.27 mmol), conc. $\mathrm{HCl}(1.21 \mathrm{~mL}, 14.54 \mathrm{mmol}), \mathrm{NaNO}_{2}$ (0.60 g, $\left.8.72 \mathrm{mmol}\right), \mathrm{KOH}(0.82 \mathrm{~g}, 14.54$ $\mathrm{mmol})$, and 3-iodophenol $(1.76 \mathrm{~g}, 8.0 \mathrm{mmol})$ were reacted according to general procedure $\mathrm{C}$, part 1. The resultant dark red solid (2.48 g, $6.18 \mathrm{mmol}), \mathrm{Cs}_{2} \mathrm{CO}_{3}(4.24 \mathrm{~g}, 12.98 \mathrm{mmol})$, and MeI $(0.46 \mathrm{~mL}, 7.42 \mathrm{mmol})$ in DMF $(15 \mathrm{~mL})$ were reacted according to general procedure C, part 2. Recrystallization from EtOH/ $\mathrm{H}_{2} \mathrm{O}$ gave 25 (1.36 g, 45\%) as fluffy orange-red needles: mp 117.5$118.1{ }^{\circ} \mathrm{C} ;{ }^{1} \mathrm{H}$ NMR $\left(\mathrm{CDCl}_{3}\right) \delta 7.82(\mathrm{dt}, J=8.7,1.8 \mathrm{~Hz}, 2 \mathrm{H}), 7.68(\mathrm{~d}, J=8.7 \mathrm{~Hz}, 1 \mathrm{H}), 7.64(\mathrm{dt}, J$ $=8.7,1.8 \mathrm{~Hz}, 2 \mathrm{H}), 7.54(\mathrm{~d}, J=1.8 \mathrm{~Hz}, 1 \mathrm{H}), 6.96(\mathrm{dd}, J=8.7,1.8 \mathrm{~Hz}, 1 \mathrm{H}) 3.21(\mathrm{~s}, 3 \mathrm{H}) ;{ }^{13} \mathrm{C}$ $\operatorname{NMR}\left(\mathrm{CDCl}_{3}\right) \delta 162.4,151.1,145.2,132.3,125.3,124.7,123.9,117.9,115.5,105.6,55.9 ; \mathrm{IR}$ (KBr) 3083, 3069, 1588, 1477, $1228 \mathrm{~cm}^{-1}$; MS (ESI) $\mathrm{m} / z 416.9\left(90, \mathrm{M}^{+}+\mathrm{H}\right), 260.8$ (25, $\left.\mathrm{M}^{+}-\mathrm{C}_{6} \mathrm{H}_{4} \mathrm{Br}\right), 183.1\left(50, \mathrm{M}^{+}-\mathrm{C}_{7} \mathrm{H}_{7} \mathrm{IO}\right)$.

(4-Fluorophenyl)(2-iodo-4-methoxyphenyl)diazene (26). 4-Fluoroaniline (2.0 g, 18.0 mmol), conc. $\mathrm{HCl}(3.0 \mathrm{~mL}, 36.0 \mathrm{mmol}), \mathrm{NaNO}_{2}(1.50 \mathrm{~g}, 21.6 \mathrm{mmol}), \mathrm{KOH}(2.02 \mathrm{~g}, 36.0 \mathrm{mmol})$, and 3-iodophenol (4.36 g, $19.8 \mathrm{mmol}$ ) were reacted according to general procedure $\mathrm{C}$, part 1 . The resultant dark red solid (5.75 g, $16.2 \mathrm{mmol}), \mathrm{Cs}_{2} \mathrm{CO}_{3}(11.8 \mathrm{~g}, 34.02 \mathrm{mmol})$, and $\mathrm{MeI}(1.21$ $\mathrm{mL}, 19.5 \mathrm{mmol})$ in DMF (36 mL) were reacted according to general procedure C, part 2 . Recrystallization from $\mathrm{EtOH} / \mathrm{H}_{2} \mathrm{O}$ afforded 26 ( $4.2 \mathrm{~g}, 66 \%$ yield) as orange needles: $\mathrm{mp}$ 94.8$95.7{ }^{\circ} \mathrm{C} ;{ }^{1} \mathrm{H} \mathrm{NMR}\left(\mathrm{CDCl}_{3}\right) \delta 7.96(\mathrm{tt}, J=5.4,3.3 \mathrm{~Hz}, 2 \mathrm{H}), 7.67(\mathrm{~d}, J=9.3 \mathrm{~Hz}, 1 \mathrm{H}), 7.53(\mathrm{~d}, J=$ $2.4 \mathrm{~Hz}, 1 \mathrm{H}), 7.19$ (tt, $J=5.4,3.3 \mathrm{~Hz}, 2 \mathrm{H}), 6.96(\mathrm{dd}, J=9.0,2.7 \mathrm{~Hz}, 1 \mathrm{H}), 3.87(\mathrm{~s}, 3 \mathrm{H}),{ }^{13} \mathrm{C} \mathrm{NMR}$ $\left(\mathrm{CDCl}_{3}\right) \delta 164.6(\mathrm{~d}, J=1002.0 \mathrm{~Hz}) 162.4,148.9(\mathrm{~d}, J=12.0 \mathrm{~Hz}), 145.1,125.2(\mathrm{~d}, J=32.1 \mathrm{~Hz})$, 123.8, 117.8, 116.1 (d, $J=92.1 \mathrm{~Hz}), 115.4,105.2,55.8$; IR (KBr) 3004, 2927, 1584, $1496 \mathrm{~cm}^{-1}$; MS (ESI) $m / z 357.0\left(100, \mathrm{M}^{+}+\mathrm{H}\right), 261.1\left(30, \mathrm{M}^{+}-\mathrm{C}_{6} \mathrm{H}_{5} \mathrm{~F}\right), 231.0\left(20, \mathrm{M}^{+}-\mathrm{I}^{125}\right), 229.3(35$, $\left.\mathrm{M}^{+}-\mathrm{I}^{127}\right)$.

(4-Carbomethoxyphenyl)(2-iodo-4-methoxyphenyl)diazene (27). Methyl 4aminobenzoate (1.25 g, $8.27 \mathrm{mmol})$, conc. $\mathrm{HCl}(1.37 \mathrm{~mL}, 16.54 \mathrm{mmol}) \mathrm{NaNO}_{2}(0.68 \mathrm{~g}, 9.92$ 
$\mathrm{mmol}), \mathrm{KOH}(0.93 \mathrm{~g}, 16.54 \mathrm{mmol})$, and 3-iodophenol (2.0 g, $9.20 \mathrm{mmol})$ were reacted according to general procedure $\mathrm{C}$, part 1 . The resultant dark red solid $(2.93 \mathrm{~g}, 7.67 \mathrm{mmol}), \mathrm{Cs}_{2} \mathrm{CO}_{3}(5.75 \mathrm{~g}$, $17.63 \mathrm{mmol})$, and $\mathrm{MeI}(0.57 \mathrm{~mL}, 9.20 \mathrm{mmol})$ in $\mathrm{DMF}(17 \mathrm{~mL})$ were reacted according to general procedure C, part 2. Recrystallization from $\mathrm{EtOH} / \mathrm{H}_{2} \mathrm{O}$ furnished 27 (2.21g, 69\%) as red needles: mp 164.5-165.4 ${ }^{\circ} \mathrm{C} ;{ }^{1} \mathrm{H}$ NMR $\left(\mathrm{CDCl}_{3}\right) \delta 8.20(\mathrm{dt}, J=8.7,1.8 \mathrm{~Hz}, 2 \mathrm{H}), 8.01(\mathrm{dt}, J=8.7,1.8 \mathrm{~Hz}$, 2H), $7.74(\mathrm{~d}, J=8.7 \mathrm{~Hz}, 1 \mathrm{H}), 7.58(\mathrm{~d}, J=2.7 \mathrm{~Hz}, 1 \mathrm{H}), 7.00(\mathrm{dd}, J=8.7,2.7 \mathrm{~Hz}, 1 \mathrm{H}), 3.98(\mathrm{~s}$, 3H), $3.91(\mathrm{~s}, 3 \mathrm{H}) ;{ }^{13} \mathrm{C} \mathrm{NMR}\left(\mathrm{CDCl}_{3}\right) \delta$ 166.6, 162.8, 154.8, 145.4, 131.6, 139.6, 124.0, 123.1, 118.0, 115.5, 106.3, 55.9, 52.3; IR (KBr) 3096, 2939, 1713, $1591 \mathrm{~cm}^{-1}$; MS (APCI) $\mathrm{m} / z(\%)$ $379.0\left(80, \mathrm{M}^{+}+\mathrm{H}\right) 269.2\left(10, \mathrm{M}^{+}-\mathrm{I}\right), 260.8\left(35, \mathrm{M}^{+}-\mathrm{C}_{8} \mathrm{H}_{8} \mathrm{O}_{2}\right), 135.1\left(100, \mathrm{M}^{+}-\mathrm{C}_{8} \mathrm{H}_{8} \mathrm{IO}_{2}\right)$.

(4-Cyanophenyl)(2-iodo-4-methoxyphenyl)diazene (28). 4-Aminobenzonitrile (2.0 g, 16.9 mmol), conc. $\mathrm{HCl}$ (2.82 mL, $33.8 \mathrm{mmol}), \mathrm{NaNO}_{2}$ (1.40 g, $\left.20.28 \mathrm{mmol}\right), \mathrm{KOH}(1.9 \mathrm{~g}, 33.8$ $\mathrm{mmol})$, and 3-iodophenol $(4.10 \mathrm{~g}, 18.60 \mathrm{mmol})$ were reacted according to general procedure $\mathrm{C}$, part 1. The resultant dark red solid (5.87 g, $16.78 \mathrm{mmol}), \mathrm{Cs}_{2} \mathrm{CO}_{3}(13.4 \mathrm{~g}, 41.4 \mathrm{mmol})$, and $\mathrm{MeI}$ $(1.15 \mathrm{~mL}, 18.46 \mathrm{mmol})$ in DMF $(34 \mathrm{~mL})$ were reacted according to general procedure C, part 2. Recrystallization from EtOH/ $\mathrm{H}_{2} \mathrm{O}$ gave $28(3.23 \mathrm{~g}, 53 \%)$ as orange needles: ${ }^{1} \mathrm{H} \mathrm{NMR}\left(\mathrm{CDCl}_{3}\right) \delta$ $8.01(\mathrm{dt}, J=6.9,1.8 \mathrm{~Hz}, 2 \mathrm{H}), 7.81(\mathrm{dt}, J=6.9,1.8 \mathrm{~Hz}, 2 \mathrm{H}), 7.73(\mathrm{~d}, J=9.0 \mathrm{~Hz}, 1 \mathrm{H}), 7.57(J=$ $2.7 \mathrm{~Hz}, 1 \mathrm{H}), 6.98(J=9.0,2.7 \mathrm{~Hz}, 1 \mathrm{H}), 3.90(\mathrm{~s}, 3 \mathrm{H}) ;{ }^{13} \mathrm{C} \mathrm{NMR}\left(\mathrm{CDCl}_{3}\right) \delta$ 163.4, 154.5, 145.4, 133.5, 124.3, 124.0, 118.9, 118.3, 115.9, 113.9, 107.2, 56.2; IR (KBr) 3017, 2976, 2938, 2225, $1479,1432 \mathrm{~cm}^{-1}$; MS (APCI) $m / z(\%) 364.0\left(40, \mathrm{M}^{+}+\mathrm{H}\right) 235.9\left(30, \mathrm{M}^{+}-\mathrm{I}^{127}\right), 129.9(100$, $\left.\mathrm{M}^{+}-\mathrm{C}_{7} \mathrm{H}_{7} \mathrm{IO}\right)$.

(2-Iodo-4-methoxyphenyl)(4-nitrophenyl)diazene (29). 4-Nitroaniline (1.5 g, 10.86 mmol), conc. $\mathrm{HCl}(1.80 \mathrm{~mL}, 21.7 \mathrm{mmol}), \mathrm{NaNO}_{2}(0.90 \mathrm{~g}, 13.03 \mathrm{mmol}), \mathrm{KOH}(1.22 \mathrm{~g}, 21.7$ $\mathrm{mmol})$, and 3-iodophenol (2.63 g, $11.95 \mathrm{mmol})$ were reacted according to general procedure $\mathrm{C}$, part 1. The resultant dark red solid (3.5 g, $9.48 \mathrm{mmol}), \mathrm{Cs}_{2} \mathrm{CO}_{3}(7.56 \mathrm{~g}, 23.23 \mathrm{mmol})$, and MeI $(0.65 \mathrm{~mL}, 10.43 \mathrm{mmol})$ in DMF $(21 \mathrm{~mL})$ were reacted according to general procedure C, part 2 . Recrystallization from EtOH/ $\mathrm{H}_{2} \mathrm{O}$ gave 29 (1.91 g, 53\%) as a dark red powder: mp 127.5-128.6 ${ }^{\circ} \mathrm{C} ;{ }^{1} \mathrm{H} \mathrm{NMR}\left(\mathrm{CDCl}_{3}\right) \delta 8.38(\mathrm{dt}, J=5.4,1.8 \mathrm{~Hz}, 2 \mathrm{H}), 8.06(\mathrm{dt}, J=5.4,1.8 \mathrm{~Hz}, 2 \mathrm{H}), 7.75(\mathrm{~d}, J=$ 
$9.0 \mathrm{~Hz}, 1 \mathrm{H}), 7.58(\mathrm{~d}, J=2.7 \mathrm{~Hz}, 1 \mathrm{H}) 7.00(\mathrm{dd}, J=9.0,2.7 \mathrm{~Hz}, 1 \mathrm{H}), 3.91(\mathrm{~s}, 3 \mathrm{H}) ;{ }^{13} \mathrm{C} \mathrm{NMR}$ $\left(\mathrm{CDCl}_{3}\right) \delta 163.3,155.38,148.5,145.2,124.8,124.1,123.8,118.1,115.6,107.3,56.0 ; \mathrm{IR}(\mathrm{KBr})$ 3094, 2910, 1587, $1481 \mathrm{~cm}^{-1}$; MS (APCI) $m / z(\%) 383.9\left(100, \mathrm{M}^{+}+\mathrm{H}\right), 338.0\left(80, \mathrm{M}^{+}-\mathrm{NO}_{2}\right)$, $150.1\left(20, \mathrm{M}^{+}-\mathrm{C}_{7} \mathrm{H}_{6} \mathrm{IO}\right)$.

[4-Methoxy-2-(triethylsilylethynyl)phenyl]phenyldiazene (30). Iododiazene 24 (0.75 g, $2.21 \mathrm{mmol})$, TESA (0.56 mL, $3.10 \mathrm{mmol}), \mathrm{PdCl}_{2}\left(\mathrm{PPh}_{3}\right)_{2}(0.03 \mathrm{~g}, 0.044 \mathrm{mmol})$, and $\mathrm{CuI}(0.042 \mathrm{~g}$, $0.221 \mathrm{mmol})$ were reacted according to general procedure D. Chromatography on silica gel (10$20 \% \mathrm{CH}_{2} \mathrm{Cl}_{2}$ gradient in hexanes) yielded $\mathbf{3 0}(0.520 \mathrm{~g}, 67 \%)$ as a viscous red oil: ${ }^{1} \mathrm{H} \mathrm{NMR}$ $\left(\mathrm{CDCl}_{3}\right)$ 7.97-7.94 (m, 2H), $7.76(\mathrm{~d}, J=9.0 \mathrm{~Hz}, 1 \mathrm{H}), 7.51-7.43(\mathrm{~m}, 3 \mathrm{H}), 7.12(\mathrm{~d}, J=2.7 \mathrm{~Hz}, 1 \mathrm{H})$, $6.94(\mathrm{dd}, J=9.0 \mathrm{~Hz}, 2.7 \mathrm{~Hz}, 1 \mathrm{H}), 3.89$ (s, 3H), 1.07 (t, $J=8.1 \mathrm{~Hz}, 9 \mathrm{H}), 0.72(\mathrm{q}, J=8.1 \mathrm{~Hz}, 6 \mathrm{H})$; ${ }^{13} \mathrm{C} \mathrm{NMR}\left(\mathrm{CDCl}_{3}\right) \delta 161.3,147.78,130.6,128.9,126.0,123.1,122.3,117.1,116.9,116.4$, 103.1, 98.7, 55.7, 7.6, 4.4; IR (neat) 2955, 2910, 2154, 1596, $1461 \mathrm{~cm}^{-1}$; MS (ESI) $m / z(\%)$ $351.3\left(100, \mathrm{M}^{+}+\mathrm{H}\right) 323.2\left(30, \mathrm{M}^{+}-\mathrm{C}_{2} \mathrm{H}_{3}\right), 309.2\left(40, \mathrm{M}^{+}-\mathrm{C}_{3} \mathrm{H}_{5}\right)$.

(4-Bromophenyl)[4-methoxy-2-(triethylsilylethynyl)phenyl]diazene (31). Iododiazene 25 (1.50 g, $3.60 \mathrm{mmol})$, TESA ( $0.87 \mathrm{~mL}, 4.86 \mathrm{mmol}), \mathrm{PdCl}_{2}(\mathrm{dppf})(0.15 \mathrm{~g}, 0.18 \mathrm{mmol}), \mathrm{CuI}$ (0.07 g, $0.36 \mathrm{mmol})$, and THF/DIPA (1:1) were reacted according to general procedure D. Chromatography on silica gel $\left(9: 1\right.$ hexanes/Et $\left.{ }_{2} \mathrm{O}\right)$ afforded $\mathbf{3 1}(1.43 \mathrm{~g}, 92 \%)$ as a red viscous oil: ${ }^{1} \mathrm{H} \mathrm{NMR}\left(\mathrm{CDCl}_{3}\right) \delta 7.84(\mathrm{dt}, J=8.4,2.1 \mathrm{~Hz}, 2 \mathrm{H}), 7.76(\mathrm{~d}, J=8.4 \mathrm{~Hz}, 1 \mathrm{H}), 7.62(J=8.4,2.1 \mathrm{~Hz}$, 2H), $7.14(\mathrm{~d}, J=2.1 \mathrm{~Hz}, 1 \mathrm{H}), 6.95(\mathrm{dd}, J=8.4,2.1 \mathrm{~Hz}, 1 \mathrm{H}), 3.91(\mathrm{~s}, 3 \mathrm{H}), 1.10(\mathrm{t}, J=7.8 \mathrm{~Hz}$, 9H), 0.74 (q, $J=7.8 \mathrm{~Hz}, 6 \mathrm{H}) ;{ }^{13} \mathrm{C} \mathrm{NMR}\left(\mathrm{CDCl}_{3}\right) \delta$ 161.7, 151.7, 147.6, 132.7, 132.1, 126.4, 124.5, 117.1, 116.9, 116.4, 102.9, 99.0, 55.7, 7. 6, 4.4; IR (neat) 2964, 2907, 2155, 1596, 1487 $\mathrm{cm}^{-1}$; MS (APCI) $m / z(\%) 429.0\left(60, \mathrm{M}^{+}+\mathrm{H}\right)$.

(4-Fluorophenyl)[4-methoxy-2-(triethylsilylethynyl)phenyl]diazene (32). Iododiazene 26 (1.50 g, $4.21 \mathrm{mmol})$, TESA (1.01 mL, $5.68 \mathrm{mmol}), \mathrm{PdCl}_{2}(\mathrm{dppf})(0.16 \mathrm{~g}, 0.19 \mathrm{mmol}), \mathrm{CuI}(0.07$ $\mathrm{g}, 0.38 \mathrm{mmol})$ and THF/DIPA (1:1) were reacted according to general procedure D. Chromatography on silica gel (7:1 hexanes: $\left.\mathrm{Et}_{2} \mathrm{O}\right)$ gave $32(1.21 \mathrm{~g}, 78 \%)$ as a viscous bright red oil: ${ }^{1} \mathrm{H}$ NMR $\left(\mathrm{CDCl}_{3}\right) \delta$ 8.02-7.95 (m, 2H), $7.75(\mathrm{~d}, J=9.0 \mathrm{~Hz}, 1 \mathrm{H}), 7.18(\mathrm{~m}, 3 \mathrm{H}), 6.95$ (dd, $J=$ 
9.0, 3.0 Hz, 1H), 3.90, (s, 3H), $1.11(\mathrm{t}, J=7.8 \mathrm{~Hz}, 9 \mathrm{H}), 0.74(\mathrm{q}, J=7.8 \mathrm{~Hz}, 6 \mathrm{H}) ;{ }^{13} \mathrm{C} \mathrm{NMR}$ $\left(\mathrm{CDCl}_{3}\right) \delta 164.2(\mathrm{~d}, J=999.0 \mathrm{~Hz}) 161.4,149.5(\mathrm{~d}, J=15.9 \mathrm{~Hz}) 147.6,126.0,125.0(\mathrm{~d}, J=31.8$ Hz), 117.1, 116.9, 116.4, 115.7 (d, $J=88.2), 103.0,98.8,55.7,7.55,4.4$; IR (KBr) 3004, 2830, 1548, $1496 \mathrm{~cm}^{-1}$; MS (ESI) $m / z 369.1\left(100, \mathrm{M}^{+}+\mathrm{H}\right)$.

(4-Carbomethoxyphenyl)[4-methoxy-2-(triethylsilylethynyl)phenyl]diazene (33). Iododiazene 27 (1.50 g, $3.79 \mathrm{mmol})$, TESA (0.91 mL, $5.12 \mathrm{mmol}), \mathrm{PdCl}_{2}(\mathrm{dppf})(0.15 \mathrm{~g}, 0.190$ $\mathrm{mmol})$, and $\mathrm{CuI}(0.07 \mathrm{~g}, 0.38 \mathrm{mmol})$ in THF/DIPA (1:1) were reacted according to general procedure D. Recrystallization from EtOH/ $\mathrm{H}_{2} \mathrm{O}$ furnished 33 (1.34 g, 86\%) as a dark red solid: mp 54.4-55.2 ${ }^{\circ} \mathrm{C} ;{ }^{1} \mathrm{H}$ NMR $\left(\mathrm{CDCl}_{3}\right) \delta 8.17(\mathrm{dt}, J=8.7,1.8 \mathrm{~Hz}, 2 \mathrm{H}), 8.00(\mathrm{dt}, J=8.7,1.8 \mathrm{~Hz}$, 2H), $7.79(\mathrm{~d}, J=8.7 \mathrm{~Hz}, 1 \mathrm{H}), 7.15(\mathrm{~d}, J=2.2 \mathrm{~Hz}, 1 \mathrm{H}), 6.96(\mathrm{dd}, J=8.7,2.2 \mathrm{~Hz}, 1 \mathrm{H}), 3.98(\mathrm{~s}$, $3 \mathrm{H}), 3.91(\mathrm{~s}, 3 \mathrm{H}), 1.10(\mathrm{t}, J=7.8 \mathrm{~Hz}, 9 \mathrm{H}), 0.76(\mathrm{q}, J=7.8 \mathrm{~Hz}, 6 \mathrm{H}) ;{ }^{13} \mathrm{C} \mathrm{NMR}\left(\mathrm{CDCl}_{3}\right) \delta 166.6$, $162.0,155.4,147.7,131.3,130.4,126.9,122.9,117.1,117.0,116.4,102.8,99.3,55.7,52.3,7.3$, 4.4; IR (KBr) 2942, 2870, 2151, 2067, 1723, $1590 \mathrm{~cm}^{-1}$; MS (APCI) $m / z(\%) 409.2$ (100, $\left.\mathrm{M}^{+}+\mathrm{H}\right), 394.1\left(15, \mathrm{M}^{+}-\mathrm{CH}_{3}\right), 351.1\left(20, \mathrm{M}^{+}-\mathrm{CO}_{2} \mathrm{Me}\right)$;

(4-Cyanophenyl)[4-methoxy-2-(triethylsilylethynyl)phenyl]diazene (34). Iododiazene 28 (1.0 g, $2.75 \mathrm{mmol})$, TESA (0.69 mL, $3.86 \mathrm{mmol}), \mathrm{PdCl}_{2}(\mathrm{dppf})(0.05 \mathrm{~g}, 0.07 \mathrm{mmol})$, and CuI (0.03 g, $0.14 \mathrm{mmol})$ in THF/DIPA (1:1) were reacted according to general procedure D.

Chromatography on silica gel (4:1 hexanes/ $\left.\mathrm{Et}_{2} \mathrm{O}\right)$ gave $34(0.721 \mathrm{~g}, 66 \%)$ as a bright orange-red solid: $\mathrm{mp} 80.9-81.5{ }^{\circ} \mathrm{C} ;{ }^{1} \mathrm{H} \mathrm{NMR}\left(\mathrm{CDCl}_{3}\right) \delta 8.01(\mathrm{dt}, J=8.4,1.8 \mathrm{~Hz}, 2 \mathrm{H}), 7.81-7.77(\mathrm{~m}, 3 \mathrm{H})$, $7.15(\mathrm{~d}, J=3.0 \mathrm{~Hz}, 1 \mathrm{H}), 6.96(\mathrm{dd}, J=9.0,3.0 \mathrm{~Hz}, 1 \mathrm{H}), 3.92(\mathrm{~s}, 3 \mathrm{H}), 1.10(\mathrm{t}, J=7.8 \mathrm{~Hz}, 9 \mathrm{H})$, $0.74(\mathrm{q}, J=7.8 \mathrm{~Hz}) ;{ }^{13} \mathrm{C} \mathrm{NMR}\left(\mathrm{CDCl}_{3}\right) \delta 162.4,154.9,147.4,133.0,127.4,123.5,118.6,117.2$, 117.01, 116.5, 113.4, 102.6, 99.7, 55.8, 7.6, 4.4; IR (KBr) 2951, 2872, 2218, 2150, $1593 \mathrm{~cm}^{-1}$; MS (ESI) $m / z(\%) 376.1\left(100, \mathrm{M}^{+}+\mathrm{H}\right), 348.1\left(30, \mathrm{M}^{+}-\mathrm{C}_{2} \mathrm{H}_{3}\right), 334.1\left(40, \mathrm{M}^{+}-\mathrm{C}_{3} \mathrm{H}_{5}\right)$.

[4-Methoxy-2-(triethylsilylethynyl)phenyl](4-nitrophenyl)diazene (35). Iododiazene 29 (0.75 g, $1.96 \mathrm{mmol})$, TESA (0.49 mL, $2.74 \mathrm{mmol}), \mathrm{PdCl}_{2}$ (dppf) (0.08 g, $\left.0.1 \mathrm{mmol}\right)$, and CuI $(0.04 \mathrm{~g}, 0.196 \mathrm{mmol})$ in THF/DIPA $(1: 1)$ were reacted according to general procedure D. Chromatography on silica gel (4:1 hexanes/ $\left.\mathrm{Et}_{2} \mathrm{O}\right)$ afforded $35(0.503 \mathrm{~g}, 65 \%)$ as a bright orange- 
red solid: $\mathrm{mp} 81.8-83.6{ }^{\circ} \mathrm{C} ;{ }^{1} \mathrm{H} \mathrm{NMR}\left(\mathrm{CDCl}_{3}\right) \delta 8.34(\mathrm{dt}, J=9.0,2.4 \mathrm{~Hz}, 2 \mathrm{H}), 8.04(\mathrm{dt}, J=9.0$, $2.4 \mathrm{~Hz}, 2 \mathrm{H}), 7.80(\mathrm{~d}, J=9.3 \mathrm{~Hz}, 1 \mathrm{H}), 7.15(\mathrm{~d}, J=2.7 \mathrm{~Hz}, 1 \mathrm{H}), 6.95(\mathrm{dd}, J=9,3,2.7 \mathrm{~Hz}, 1 \mathrm{H})$, 3.92 (s, 3H), 1.08 (t, J=7.8 Hz, 9H), 0.74 (q, J=7.8 Hz, 6H); ${ }^{13} \mathrm{C}$ NMR $\left(\mathrm{CDCl}_{3}\right) \delta 162.7,156.1$, $148.4,147.5,127.7,124.6,123.5,117.2,117.1,116.6,102.6,100.0,55.8,7.6,4.4$; IR (KBr) 2955, 2912, 2155, 2064, $1591 \mathrm{~cm}^{-1}$; (ESI) $\mathrm{m} / z(\%) 396.1\left(100, \mathrm{M}^{+}+\mathrm{H}\right)$.

3-Hydroxymethyl-2-phenyl-2H-indazole (36). Diazene 8 (0.166 g, $0.52 \mathrm{mmol})$ was deprotected with TBAF (1.04 mL, $1.04 \mathrm{mmol})$, cyclized with TESOH (0.20 mL, $1.3 \mathrm{mmol})$, and desilylated with xs TBAF according to general procedure E. After aqueous work-up, filtration over silica (EtOAc) and concentration in vacuo afforded isoindazole 36 (67 $\mathrm{mg}, 75 \%)$ as a light tan solid: $\mathrm{mp} 150.5-151.8{ }^{\circ} \mathrm{C} ;{ }^{1} \mathrm{H}$ NMR $\left(\mathrm{CDCl}_{3}\right) \delta$ 7.76-7.67 (m, 4H), 7.54-7.46 (m, 3H), 7.34 $(\mathrm{td}, J=8.1,0.9 \mathrm{~Hz}, 1 \mathrm{H}), 7.12(\mathrm{td}, J=8.1,0.9 \mathrm{~Hz}, 1 \mathrm{H}), 4.96(\mathrm{~s}, 2 \mathrm{H}), 2.56(\mathrm{br} \mathrm{s}, 1 \mathrm{H}) ;{ }^{13} \mathrm{C} \mathrm{NMR}$ $\left(\mathrm{CDCl}_{3}\right) \delta 148.5,139.4,133.8,129.2,128.9,126.9,125.4,122.2,121.8,119.4,117.8,54.1 ; \mathrm{IR}$ (KBr) 3183, 3060, 1595, $1503 \mathrm{~cm}^{-1}$; MS (ESI) $m / z$ (\%) $225.1\left(100, \mathrm{M}^{+}+\mathrm{H}\right), 207.2\left(35, \mathrm{M}^{+}-\mathrm{OH}\right)$, $149.9\left(100, \mathrm{M}^{+}-\mathrm{N}_{2}\right)$.

3-Hydroxymethyl-5-methyl-2-phenyl-2H-indazole (37). Diazene 14 (0.233 g, 0.70 mmol) was deprotected with TBAF (1.4 mL, $1.4 \mathrm{mmol})$, cyclized with TESOH (0.32 mL, $2.09 \mathrm{mmol})$ and desilylated with xs TBAF according to general procedure E. After aqueous work-up, filtration over silica (EtOAc) and concentration in vacuo afforded isoindazole 37 (0.135 g, 81\%) as a light yellow solid: mp 164.4-165.5 ${ }^{\circ} \mathrm{C} ;{ }^{1} \mathrm{H}$ NMR $\left(\mathrm{CDCl}_{3}\right) \delta$ 7.69-7.65 (m, 3H), 7.52-7.42 (m, 4H), $7.17(\mathrm{dd}, J=9.01 .5 \mathrm{~Hz}, 1 \mathrm{H}), 4.92(\mathrm{~s}, 2 \mathrm{H}), 2.56(\mathrm{br} \mathrm{s}, 1 \mathrm{H}), 2.42(\mathrm{~s}, 3 \mathrm{H}) ;{ }^{13} \mathrm{C} \mathrm{NMR}\left(\mathrm{CDCl}_{3}\right)$ $\delta 147.5,139.5,132.9,131.7,129.8,129.2,128.7,125.4,122.1,117.4,117.4,54.0,21.8 ;$ IR (KBr) 3215, 3054, 2916, 1535, $1504 \mathrm{~cm}^{-1}$; MS (ESI) $m / z(\%) 239.1\left(65, \mathrm{M}^{+}+\mathrm{H}\right), 221.1(35$, $\left.\mathrm{M}^{+}-\mathrm{OH}\right)$.

5-tert-Butyl-3-hydroxymethyl-2-phenyl-2H-indazole (38). Diazene 15 (0.143 g, 0.378 mmol) was deprotected with TBAF $(0.756 \mathrm{~mL}, 0.756 \mathrm{mmol})$, cyclized with TESOH $(0.144 \mathrm{~mL}$, $0.945 \mathrm{mmol}$ ), and desilylated with xs TBAF according to general procedure E. After aqueous work-up, filtration over silica (EtOAc) and concentration in vacuo furnished isoindazole 38 (63 
mg, 80\%) as a tan solid: $\mathrm{mp} 178.4-179.7{ }^{\circ} \mathrm{C} ;{ }^{1} \mathrm{H} \operatorname{NMR}\left(\mathrm{CDCl}_{3}\right) \delta 7.75-7.70(\mathrm{~m}, 3 \mathrm{H}), 7.62(\mathrm{~d}, J=$ $0.9 \mathrm{~Hz}, 1 \mathrm{H}), 7.56-7.46(\mathrm{~m}, 4 \mathrm{H}), 5.00(\mathrm{~s}, 2 \mathrm{H}), 2.10($ br s, $1 \mathrm{H}), 1.39(\mathrm{~s}, 9 \mathrm{H}) ;{ }^{13} \mathrm{C} \mathrm{NMR}\left(\mathrm{CDCl}_{3}\right) \delta$ $147.4,145.2,139.6,133.5,129.3,128.8,126.8,125.5,121.7,117.5,113.3,54.1,35.0,31.2$; IR (KBr) 3216, 2957, 2858, 1596, $1503 \mathrm{~cm}^{-1}$; MS (ESI) $m / z$ (\%) $281.3\left(100, \mathrm{M}^{+}+\mathrm{H}\right), 263.1$ (15, $\left.\mathrm{M}^{+}-\mathrm{OH}\right), 235.1\left(20, \mathrm{M}^{+}-\mathrm{C}_{3} \mathrm{H}_{9}\right)$.

5-Chloro-3-hydroxymethyl-2-phenyl-2H-indazole (39). Diazene 27 (0.50 g, 1.40 mmol), was deprotected with TBAF (2.8 mL, $2.8 \mathrm{mmol})$, cyclized with TESOH (0.68 mL, $4.2 \mathrm{mmol})$, and desilylated with xs TBAF according to general procedure E. After aqueous work-up, filtration over silica (EtOAc) and concentration in vacuo afforded isoindazole 39 (0.296 g, 82\%) as a dark brown solid: $\mathrm{mp} 172.4-173.7{ }^{\circ} \mathrm{C} ;{ }^{1} \mathrm{H} \mathrm{NMR}\left(\mathrm{CDCl}_{3}\right) \delta$ 7.75-7.68 (m, 4H) 7.58-7.50 (m, 3H), $7.27(\mathrm{dd}, J=9.0,2.1 \mathrm{~Hz}, 1 \mathrm{H}) 4.97(\mathrm{~s}, 2 \mathrm{H}), 2.07$ (br s, $1 \mathrm{H}) ;{ }^{13} \mathrm{C} \mathrm{NMR}\left(\mathrm{CDCl}_{3}\right) \delta 158.5$, $152.1,139.3,136.1,129.4,129.2,128.3,128.0,125.4,119.5,118.3,54.3$; IR (KBr) 3218, 3063, 2920, 1596, $1501 \mathrm{~cm}^{-1}$; MS (ESI) $m / z(\%) 259.0\left(85, \mathrm{M}^{+}+\mathrm{H}\right), 241.1\left(45, \mathrm{M}^{+}-\mathrm{OH}\right)$,

5-Ethynyl-3-hydroxymethyl-2-phenyl-2H-indazole (40). Diazene 30 (0.34 g, 0.74 mmol) was deprotected with TBAF $(2.96 \mathrm{~mL}, 2.96 \mathrm{mmol})$, cyclized with TESOH $(0.36 \mathrm{~mL}, 2.22$ mmol), and desilylated with xs TBAF according to general procedure E. After aqueous work-up, filtration over silica (EtOAc) and concentration in vacuo afforded isoindazole 40 (0.147 g, 80\%) as a yellow solid: $\mathrm{mp} 136.2-137.4{ }^{\circ} \mathrm{C} ;{ }^{1} \mathrm{H} \mathrm{NMR}\left(\mathrm{CDCl}_{3}\right) \delta 7.88(\mathrm{~s}, 1 \mathrm{H}), 7.67-7.64(\mathrm{~m}, 3 \mathrm{H})$, 7.53$7.42(\mathrm{~m}, 3 \mathrm{H}), 7.37(\mathrm{~d}, J=9.0 \mathrm{~Hz}, 1 \mathrm{H}), 4.91(\mathrm{~s}, 2 \mathrm{H}), 3.07(\mathrm{~s}, 1 \mathrm{H}), 2.64(\mathrm{br} \mathrm{s}, 1 \mathrm{H}) ;{ }^{13} \mathrm{C} \mathrm{NMR}$ $\left(\mathrm{CDCl}_{3}\right) \delta 147.7,139.1,134.4,130.0,129.3,129.2,125.3,124.6,121.4,117.9,115.7,84.4,76.5$, 54.1; IR (KBr) 3298, 3235, 2924, 2101, 1598, $1504 \mathrm{~cm}^{-1}$; MS (ESI) $m / z(\%) 249.1\left(\mathrm{M}^{+}+\mathrm{H}\right)$, $231.1\left(30, \mathrm{M}^{+}-\mathrm{OH}\right), 219.1\left(\mathrm{M}^{+}-\mathrm{CH}_{2} \mathrm{O}\right)$.

3-Hydroxymethyl-5-methoxy-2-phenyl-2H-indazole (41). Diazene 30 (0.41 g, 1.17 mmol) was deprotected with TBAF $(2.34 \mathrm{~mL}, 2.34 \mathrm{mmol})$, cyclized with TESOH $(0.54 \mathrm{~mL}, 3.51$ mmol), and desilylated with xs TBAF according to general procedure E. After aqueous work-up, filtration over silica (EtOAc) and concentration in vacuo afforded isoindazole $41(0.231 \mathrm{~g}, 79 \%)$ as a light brown solid: $\mathrm{mp} 184.4-185.4{ }^{\circ} \mathrm{C} ;{ }^{1} \mathrm{H} \mathrm{NMR}\left(\mathrm{CDCl}_{3}\right) \delta$ 7.73-7.66 (m, 3H), 7.56-7.48 (m, 
3H), $7.07(\mathrm{dd}, J=9.0,2.1 \mathrm{~Hz}, 1 \mathrm{H}), 6.95(\mathrm{~d}, J=2.1 \mathrm{~Hz}, 1 \mathrm{H}), 4.96(\mathrm{~s}, 2 \mathrm{H}), 3.87(\mathrm{~s}, 3 \mathrm{H}), 1.72(\mathrm{br}$

$\mathrm{s}, 1 \mathrm{H}) ;{ }^{13} \mathrm{C} \mathrm{NMR}\left(\mathrm{CDCl}_{3}\right) \delta 155.5,145.4,139.6,132.6,129.2,128.7,125.3,122.0,121.9,119.3$, 95.3, 55.4, 54.2; IR (KBr) 3218, 3051, 2826, 1537, $1506 \mathrm{~cm}^{-1}$; MS (APCI) $m / z$ (\%) 255.1 (100, $\left.\mathrm{M}^{+}+\mathrm{H}\right), 237.2\left(30, \mathrm{M}^{+}-\mathrm{OH}\right)$.

2-(4-Bromophenyl)-3-hydroxymethyl-5-methoxy-2H-indazole (42). Diazene 31 (0.43 g, $1.0 \mathrm{mmol})$ was desilylated with TBAF $(2.0 \mathrm{~mL}, 2.0 \mathrm{mmol})$, cyclized with TESOH $(0.487 \mathrm{~mL}$, $3.0 \mathrm{mmol}$ ) and deprotected with xs TBAF according to general procedure E. After aqueous work-up, filtration over silica $\left(\mathrm{Et}_{2} \mathrm{O}\right)$, and concentration in vacuo gave $42(0.268 \mathrm{~g}, 63 \%)$ as a light brown solid. mp 162.7-164.4 ${ }^{\circ} \mathrm{C} ;{ }^{1} \mathrm{H}$ NMR $\left(\mathrm{CDCl}_{3}\right) \delta$ 7.63-7.62 (m, 5H), 7.04 (dd, $J=9.0$, $2.1 \mathrm{~Hz}, 1 \mathrm{H}), 6.83(\mathrm{~d}, J=2.1 \mathrm{~Hz}, 1 \mathrm{H}), 4.92(\mathrm{~s}, 2 \mathrm{H}), 3.84(\mathrm{~s}, 3 \mathrm{H}), 2.38(\mathrm{br} \mathrm{s}, 1 \mathrm{H}),{ }^{13} \mathrm{C}$ NMR $\left(\mathrm{CDCl}_{3}\right) \delta 153.9,145.7,133.25,132.9,132.6,126.8,125.1,122.8,122.7,119.4,95.5,55.7$, 54.1; IR (KBr) 3203, 2923, 1637, $1516 \mathrm{~cm}^{-1}$; MS (APCI) $\mathrm{m} / z(\%) 333.1\left(100, \mathrm{M}^{+}+\mathrm{H}\right), 317.0(30$, $\left.\mathrm{M}^{+}-\mathrm{CH}_{3}\right), 319.4\left(25, \mathrm{M}^{+}-\mathrm{OH}\right)$.

2-(4-Fluorophenyl)-3-hydroxymethyl-5-methoxy-2H-indazole (43). Diazene 32 (0.46 g, $1.24 \mathrm{mmol})$ was deprotected with TBAF $(2.48 \mathrm{~mL}, 2.48 \mathrm{mmol})$, cyclized with TESOH $(0.57 \mathrm{~mL}$, $3.72 \mathrm{mmol}$ ), and desilylated with xs TBAF according to general procedure E. After aqueous work-up, filtration over silica $\left(\mathrm{Et}_{2} \mathrm{O}\right)$ and concentration in vacuo furnished $43(0.264 \mathrm{~g}, 78 \%)$ as a light brown solid: $\mathrm{mp} 191.6-192.2{ }^{\circ} \mathrm{C} ;{ }^{1} \mathrm{H}$ NMR $\left(\mathrm{CDCl}_{3}\right) \delta$ 7.75-7.70 (m, 2H), $7.64(\mathrm{~d}, J=9.6$ Hz, 1H), 7.24-7.18 (m, 2H), 7.05 (dd, $J=9.6,2.4 \mathrm{~Hz}, 1 \mathrm{H}), 6.89$ (d, $J=2.4 \mathrm{~Hz}, 1 \mathrm{H}), 4.95$ (s, 2H),

3.86 (s, 3H), 2.20 (br s, $1 \mathrm{H}) ;{ }^{13} \mathrm{C} \mathrm{NMR}\left(\mathrm{CDCl}_{3}\right) \delta 162.5$ (d, $\left.J=990 \mathrm{~Hz}\right), 155.7,145.4,132.5$ (d, $J$ $=30.6 \mathrm{~Hz}), 127.1(\mathrm{~d}, J=32.1 \mathrm{~Hz}), 126.6,122.2,121.9,119.3,116.2(\mathrm{~d}, J=92.1 \mathrm{~Hz}), 95.2,55.4$, 54.0; IR (KBr) 3203, 2923, $1516 \mathrm{~cm}^{-1}$; MS (APCI) $\mathrm{m} / z$ (\%) $273.2\left(100, \mathrm{M}^{+}+\mathrm{H}\right), 255.1$ (25, $\left.\mathrm{M}^{+}-\mathrm{OH}\right) ; 243.1\left(45, \mathrm{M}^{+}-\mathrm{CHO}\right)$.

4-(3-Hydroxymethyl-5-methoxy-indazol-2-yl)-benzoic acid methyl ester (44). Diazene 33 $(0.45 \mathrm{~g}, 1.10 \mathrm{mmol})$ was desilylated with TBAF $(2.20 \mathrm{~mL}, 2.20 \mathrm{mmol})$, cyclized with and TESOH $(0.50 \mathrm{~mL}, 3.3 \mathrm{mmol})$, and deprotected with $\mathrm{xs}$ TBAF according to general procedure E. After aqueous work-up, filtration over silica $\left(\mathrm{Et}_{2} \mathrm{O}\right)$, and concentration in vacuo $44(0.247 \mathrm{~g}$, 
$72 \%)$ was obtained as a light brown solid. mp 195.4-197.3 ${ }^{\circ} \mathrm{C}(\mathrm{dec}) ;{ }^{1} \mathrm{H}$ NMR $\left(\mathrm{CDCl}_{3}, 300 \mathrm{MHz}\right)$ $\delta 8.20(\mathrm{dt}, J=8.7,2.1 \mathrm{~Hz}, 2 \mathrm{H}) 7.90(\mathrm{dt}, J=8.7,2.1 \mathrm{~Hz}, 2 \mathrm{H}), 7.65(\mathrm{~d}, J=9.3 \mathrm{~Hz}, 1 \mathrm{H}), 7.06(\mathrm{dd}$, $J=9.3,2.1 \mathrm{~Hz}, 1 \mathrm{H}), 6.89$ (d, $J=2.1 \mathrm{~Hz}, 1 \mathrm{H}), 5.00$ (s, 2H), 3.97 (s, 3H), 3.88 (s, 3H), 2.08 (bs, 1H); ${ }^{13} \mathrm{C} \mathrm{NMR}\left(\mathrm{CDCl}_{3}, 75 \mathrm{MHz}\right) \delta$ 166.3, 155.8, 145.9, 143.3, 132.4, 130.7, 129.9, 124.8, 122.7, 119.5, 95.0, 77.2, 55.4, 54.0, 52.4. IR (KBr) 3422, 2945, 1723, 1703, $1606 \mathrm{~cm}^{-1}$; MS (APCI) $\mathrm{m} / z$ (\%) $313.1\left(100, \mathrm{M}^{+}+\mathrm{H}\right), 295.0\left(35, \mathrm{M}^{+}-\mathrm{OH}\right), 236.1\left(40, \mathrm{M}^{+}-\mathrm{C}_{6} \mathrm{H}_{4}\right)$.

2-(4-Cyanophenyl)-3-hydroxymethyl-5-methoxy-2H-indazole (45). Diazene 34 (0.395 g, $1.0 \mathrm{mmol})$ was deprotected with TBAF $(2.0 \mathrm{~mL}, 2.0 \mathrm{mmol})$, cyclized with TESOH $(0.46 \mathrm{~mL}, 3.0$ mmol), and desilylated with xs TBAF according to general procedure E. After aqueous work-up, filtration over silica $\left(\mathrm{Et}_{2} \mathrm{O}\right)$ and concentration in vacuo afforded $45(0.21 \mathrm{~g}, 73 \%)$ as a light brown solid: mp 217.0-218.8 ${ }^{\circ} \mathrm{C}(\mathrm{dec}) ;{ }^{1} \mathrm{H} \mathrm{NMR}\left(\mathrm{CDCl}_{3}\right) \delta 8.05(\mathrm{dt}, J=8.7,2.1 \mathrm{~Hz}, 2 \mathrm{H}), 7.85$ (dt, $J=8.7,2.1 \mathrm{~Hz}, 2 \mathrm{H}), 7.65(\mathrm{~d}, J=9.0,1 \mathrm{H}), 7.06(\mathrm{dd}, J=9.0,2.1 \mathrm{~Hz}, 1 \mathrm{H}), 6.87(\mathrm{~d}, J=2.1 \mathrm{~Hz}$, 1H), $5.00(\mathrm{~s}, 2 \mathrm{H}), 3.89(\mathrm{~s}, 3 \mathrm{H}), 2.01(\mathrm{br} \mathrm{s}, 1 \mathrm{H}) ;{ }^{13} \mathrm{C} \mathrm{NMR}\left(\mathrm{C}_{4} \mathrm{D}_{8} \mathrm{O}\right) \delta$ 156.7, 147.2, 144.2, 135.0, 134.1, 125.9, 123.9, 123.5, 120.2, 118.9, 112.7, 96.5 55.7, 53.6; IR (KBr) 3205, 2825, 2234, 1536, $1218 \mathrm{~cm}^{-1}$; MS (APCI) $m / z(\%) 280.2\left(100, \mathrm{M}^{+}+\mathrm{H}\right), 262.2\left(25, \mathrm{M}^{+}-\mathrm{OH}\right), 250.1(60$, $\left.\mathrm{M}^{+}-\mathrm{CHO}\right)$.

3-Hydroxymethyl-5-methoxy-2-(4-nitrophenyl)-2H-indazole (46). Diazene 35 (0.31 g, $0.80 \mathrm{mmol})$ was deprotected with TBAF $(1.6 \mathrm{~mL}, 1.6 \mathrm{mmol})$, cyclized with TESOH $(0.37 \mathrm{~mL}$, $2.39 \mathrm{mmol}$ ), and desilylated with xs TBAF according to general procedure E. After aqueous work-up, filtration over silica $\left(\mathrm{Et}_{2} \mathrm{O}\right)$ and concentration in vacuo gave $46(0.177 \mathrm{~g}, 54 \%)$ as a light brown solid: $\mathrm{mp} 204.6-205.4{ }^{\circ} \mathrm{C} ;{ }^{1} \mathrm{H}$ NMR $\left(\mathrm{CDCl}_{3}\right) \delta 8.36(\mathrm{dt}, J=9.0,2.1 \mathrm{~Hz}, 2 \mathrm{H}), 8.05$ (dt, $J=9.0,2.1 \mathrm{~Hz}, 2 \mathrm{H}), 7.63(\mathrm{~d}, J=9.3 \mathrm{~Hz}, 1 \mathrm{H}), 7.05(\mathrm{dd}, J=9.3,2.1 \mathrm{~Hz}, 1 \mathrm{H}), 6.80(\mathrm{~d}, J=2.1$ $\mathrm{Hz}, 1 \mathrm{H}), 4.98(\mathrm{~d}, J=5.1 \mathrm{~Hz}, 2 \mathrm{H}), 3.84(\mathrm{~s}, 3 \mathrm{H}), 2.51(\mathrm{t}, J=5.1 \mathrm{~Hz}, 1 \mathrm{H}) ;{ }^{13} \mathrm{C} \mathrm{NMR}\left(\mathrm{CDCl}_{3}\right) \delta$ 156.1, 146.9, 146.2, 144.5, 132.6, 125.1, 124.8, 123.4, 122., 119.4, 94.8, 55.4, 53.6; IR (KBr) 3534, 3080, 2928, $1596 \mathrm{~cm}^{-1}$; MS (ESI) $\mathrm{m} / z(\%) 300.2\left(100, \mathrm{M}^{+}+\mathrm{H}\right), 282.0\left(15, \mathrm{M}^{+}-\mathrm{OH}\right), 254.0$ $\left(60, \mathrm{M}^{+}+\mathrm{H}-\mathrm{NO}_{2}\right)$. 
5-Chloro-3-(2,2,3,3-tetramethyl-cyclopropyl)-2-phenyl-2H-indazole (47). Diazene 16 (0.34 g, $0.96 \mathrm{mmol})$ was desilylated with TBAF (1.92 mL, $1.92 \mathrm{mmol})$ according to the first step of general procedure E. Upon concentration the deprotected alkyne was dissolved in 2,3dimethyl-2-butene $(20 \mathrm{~mL})$ and purged with $\operatorname{Ar}(20 \mathrm{~min})$. The solution was placed in a preheated $75^{\circ} \mathrm{C}$ sand bath and stirred under Ar for $8 \mathrm{~h}$. After cooling, the solvent was removed and the black oil was dissolved in 5:1 hexanes $/ \mathrm{CH}_{2} \mathrm{Cl}_{2}$ and filtered over silica to yield the cyclopropane 47 (0.234 g, 75\%) as a dark brown solid: mp 157.3-158 ${ }^{\circ} \mathrm{C}(\mathrm{dec}) ;{ }^{1} \mathrm{H}$ NMR $\left(\mathrm{CDCl}_{3}\right) \delta$ 7.68-7.63 (m, 3H), 7.56-7.45 (m, 4H), $7.22(\mathrm{dd}, J=9.3,1.8 \mathrm{~Hz}, 1 \mathrm{H}), 1.72(\mathrm{~s}, 1 \mathrm{H})$, $1.20(\mathrm{~s}, 6 \mathrm{H}), 0.56(\mathrm{~s}, 6 \mathrm{H}) ;{ }^{13} \mathrm{C} \mathrm{NMR}\left(\mathrm{CDCl}_{3}\right) \delta 147.3,141.0,134.4,128.8,128.3,127.8,126.2$, 125.2, 123.7, 120.2, 119.3, 100.0, 30.0, 24.7, 23.0, 19.0; IR (KBr) 2979, 2921, 1596, $1498 \mathrm{~cm}^{-1}$; MS (APCI) m/z (\%) $325.0\left(100, \mathrm{M}^{+}+\mathrm{H}\right), 309.0\left(25, \mathrm{M}+-\mathrm{CH}_{3}\right), 241.1\left(\mathrm{M}+-\mathrm{C}_{6} \mathrm{H}_{11}\right)$. 


\section{Cartesian Coordinates for all structures in Figure 1}

4 - Conformation 1

N $0.1014500 .006610-0.177560$

C -0.346360 0.079330 - 1.516970

C - $1.0553800 .222740-4.222960$

C $0.6654000 .110040-2.508200$

C - $1.6977600 .120900-1.895440$

C $-2.0500900 .191970-3.236430$

C $0.2865700 .182220-3.862470$

C $2.0525400 .069870-2.170130$

H -2.448340 $0.095860-1.113560$

H $-3.0985600 .223720-3.520000$

H $1.0627200 .205660-4.620600$

H -1.329960 0.278350 -5.272670

$\mathrm{N}-0.815070-0.0191900 .688220$

C $3.2445500 .040130-1.964650$

H $4.2902400 .012240-1.758440$

C $-0.357830-0.0920402 .027680$

C - $1.361200-0.1219003 .005530$

C - $1.022250-0.1932304 .355640$

C $0.321180-0.2349404 .732460$

C $1.325590-0.2051603 .755720$

C $0.995550-0.1341102 .407800$

H -2.396860 -0.088080 2.681030

H -1.802860 -0.216200 5.110920

Н $0.589600-0.2905605 .784070$

H $2.370230-0.2378304 .054040$

H $1.756040-0.1099701 .635120$ 
4 Conformation 2

$\mathrm{N}-0.7263800 .0849200 .380200$

C -0.1501000 .1889901 .665700$

C 0.6543400 .4643604 .342540

C $1.210940-0.0402702 .013970$

C - 1.0727500 .5210602 .669420

C -0.6825300 .6729603 .994680$

C 1.5789200 .1053003 .365940

C $2.230190-0.4547001 .103440$

H -2.104590 0.665470 2.364950

H -1.413480 0.943980 4.750920

H 2.614610 -0.076500 3.635400

Н 0.9767400 .5695005 .374640

N $0.0843800 .108270-0.583980$

C $3.192940-0.8347500 .474690$

H $4.004830-1.159910-0.134920$

C -0.511520 -0.024000-1.863950

C $0.3753100 .059710-2.945830$

C -0.099350 -0.056110-4.251190

C - $1.461690-0.258140-4.479480$

C -2.349290 -0.344280 -3.398800

C - $1.883020-0.229140-2.094640$

H $1.4284500 .216100-2.733860$

H $0.5906300 .010330-5.087780$

H -1.835290 -0.350100 -5.495840

H -3.408920 -0.503420 - 3.581050

H -2.553400 -0.293670 - 1.244870 
Pseudocoarctate TS conformation 1

$\mathrm{N}-1.585071-0.174047-0.946318$

C - $1.006323-0.0718210 .312544$

C 0.3592660 .1295492 .698032

C $0.404754-0.0277320 .298954$

C - $1.746823-0.0166781 .502075$

C - 1.0510870 .0829182 .697315

C 1.0910730 .0749721 .517169

C $0.969942-0.077229-1.017779$

H -2.831172 -0.053974 1.465342

H - 1.5904500 .1271273 .638763

H 2.1755440 .1154201 .512598

Н 0.8849150 .2121423 .645687

$\mathrm{N}-0.659456-0.261241-1.812505$

C $2.1307060 .039067-1.582448$

H $2.3212610 .007080-2.650676$

C -0.973343 -0.292957-3.184157

C $-0.105035-0.984088-4.041447$

C -0.372841 -1.011981 -5.408865

C - $1.492840-0.353820-5.920878$

C -2.355166 0.333527-5.059736

C -2.100529 $0.370622-3.692941$

H $0.743374-1.513549$-3.624347

H 0.292064 -1.555158 -6.074232

H -1.696034 -0.376502 -6.987797

H -3.226556 0.846178 -5.457502

H -2.751062 $0.906125-3.010031$ 
Pseudocoarctate TS conformation 2

H -4.871870 - $1.917200-0.152300$

C $-4.046880-1.213430-0.096230$

C - $2.736820-1.670430-0.149960$

C -4.3103600 .1636500 .030620$

C -3.279900 1.0967900 .103860

H -5.340890 0.5060300 .072300

H - $2.496450-2.724570-0.248670$

C - 1.9503500 .6535700 .054570

C $-1.704550-0.728340-0.073530$

H -3.492590 2.1572500 .201820

$\mathrm{N}-0.356910-1.073410-0.122120$

C -0.7784101 .4762800 .122510$

N $0.344670-0.021500-0.061530$

C -0.2188402 .6252000 .243620$

H - 0.7595303 .5566300 .385160

C $1.751310-0.129430-0.032220$

C $2.5072501 .021080-0.304370$

C $3.8983400 .939090-0.282700$

C $4.532960-0.2707700 .004190$

C $3.771390-1.4124700 .280210$

C $2.383350-1.3492000 .267510$

H $1.9880001 .951540-0.500390$

H $4.4882801 .826680-0.493240$

H $5.618060-0.3270500 .017210$

H $4.264070-2.3532300 .510020$

H $1.777580-2.2213200 .485240$ 
Isoindazole carbene conformation 1

H $-0.708250-0.0022105 .219550$

C -0.2703600 .0302104 .225980$

C - $1.084970-0.0653103 .114950$

C 1.1388100 .1719804 .099840

C 1.7608300 .2235002 .863700

H 1.7381100 .2400605 .003850

H -2.162300 -0.171430 3.192740

C 0.9498000 .1230901 .723400

C $-0.453480-0.0155501 .854320$

H 2.8348200 .3355502 .755690

N -1.048320 -0.109760 0.627140

C 1.2338500 .1554200 .317040

$\mathrm{N}-0.080420-0.009030-0.255610$

C $2.4606900 .395220-0.281100$

H $2.3394900 .512840-1.373060$

C $-0.403570-0.081310-1.641120$

C $0.392160-0.838170-2.507430$

C $0.062780-0.890950-3.861960$

C $-1.053380-0.206100-4.344010$

C $-1.8510100 .532990-3.465900$

C $-1.5296500 .601020-2.112980$

H 1.231230 - $1.402720-2.117960$

H $0.675840-1.480960-4.536880$

H - $1.306250-0.253200-5.399370$

H -2.723460 $1.063600-3.836020$

H -2.133000 $1.173740-1.417510$ 
Isoindazole carbene conformation 2

H -0.848440 0.1477705 .182600

C -0.3719900 .1296704 .206720$

C -1.147110 0.048010 3.064870

C 1.0433600 .1908004 .130400

C 1.7115300 .1727302 .915410

H 1.6145300 .2516505 .052800

H -2.230860 0.0015503 .102020

C 0.9426900 .0905701 .745200

C -0.4675900 .0304501 .831260$

H 2.7958700 .2201902 .867270

$\mathrm{N}-1.025250-0.0598600 .585080$

C 1.2644700 .0543200 .341320

$\mathrm{N}-0.032500-0.042610-0.269440$

C $2.4239100 .143180-0.407830$

H 3.2677000 .1951300 .306980

C $-0.350450-0.084810-1.664940$

C $0.512330-0.688050-2.587340$

C $0.147160-0.718020-3.932720$

C - $1.063180-0.168070-4.356840$

C - $1.9192100 .424910-3.425500$

C - $1.5676300 .470870-2.079810$

H 1.459130 - 1.086310 -2.250910

H 0.816320 -1.183030 -4.650900

H - $1.339160-0.200530-5.407210$

H - $2.8614900 .859710-3.746420$

H -2.218920 $0.931880-1.346870$ 
TS (carbene conformation 2 to $\mathbf{5}$ )

H 4.2672609 .3196600 .481710

C 3.3407809 .8853500 .523740

C 2.1314109 .2146800 .547730

C -5.26110010 .6033300 .474340$

C -4.55728011 .8151900 .462770$

H -6.344760 10.6030500 .381310

H 2.0732408 .1311000 .533780

C -3.168210 11.8093700 .457840

C 0.9564509 .9913000 .601820

H -5.103800 12.7538300 .395970

$\mathrm{N}-0.3305909 .5419800 .579330$

C -2.493670 10.5895600 .603830

$\mathrm{N}-1.08172010 .6283600 .704700$

C -0.99742013 .0363000 .980560$

H - 2.26594012 .8117700 .849590

C -0.35382011 .8356800 .750290$

C 1.01482011 .4149700 .638030

C 2.25153012 .0742400 .626570

C 3.40162011 .3009100 .555780

C -4.5712009 .3904200 .585660$

C -3.1824709 .3707000 .675520$

H - 0.48585013 .9233900 .589410

H 2.31050013 .1584400 .660230

H 4.37216011 .7876200 .521510

H -5.121740 8.4545600 .622990

H -2.631950 8.4479500 .825950 
Tetracycle 5

H 4.3994659 .7094720 .611983

C 3.40109610 .1389500 .612379

C 2.3047849 .3069520 .612743

C -5.13094210 .9172800 .612329$

C -4.19998611 .9664300 .612496$

H -6.193029 11.144850 0.611887

H 2.4049848 .2259080 .612662

C - 2.84634311 .6614000 .612992

C 1.0169919 .8979580 .613214

H -4.537645 12.9996700 .612168

N -0.179900 9.261176 0.613517

C - 2.45149110 .3130500 .613414

$\mathrm{N}-1.04477310 .2860200 .614084$

C -1.61034512 .5569200 .613509$

$\mathrm{H}-1.57960513 .2117501 .494771$

C -0.50379311 .5376300 .614228$

C 0.87872111 .3399500 .613319

C 2.03132412 .1654100 .613097

C 3.26727211 .5599200 .612553

C -4.7099139 .5839510 .612727$

C -3.3494719.256428 0.613319

H - $1.57837813 .211520-0.267860$

H 1.94015513 .2483100 .613284

H 4.16588912 .1708600 .612322

H -5.448521 8.7874210 .612598

H -2.999911 8.229923 0.613639 
(Pseudo)pericyclic TS to cinnolinium zwitterion

$\mathrm{N}-0.300130-0.6250000 .187880$

C $-1.678420-0.3934300 .144620$

C $-4.467300-0.302490-0.047750$

C $-2.3175100 .838990-0.174180$

C $-2.448600-1.5557700 .366790$

C $-3.829460-1.5101700 .286600$

C $-3.7217000 .844850-0.283370$

C $-1.5295202 .022240-0.392780$

H - $1.919990-2.4747000 .603810$

H $-4.417170-2.4050500 .469890$

H -4.204230 $1.782630-0.542470$

H -5.550910 -0.268450 - 0.124180

N 0.5009400 .3565700 .152870

C $-0.2717602 .117370-0.291280$

H $0.6106202 .727210-0.307210$

C 1.8805200 .0235400 .078520

C 2.7949400 .9638400 .571960

C 4.1622000 .7044000 .499220

C $4.620280-0.482650-0.076490$

C $3.705200-1.416010-0.574950$

C $2.337830-1.169720-0.500440$

H 2.4259501 .8736001 .035630

H 4.8695001 .4287400 .893060

H $5.686750-0.679740-0.140370$

H $4.061700-2.337180-1.027920$

H $1.612000-1.878070-0.884190$ 
Cinnolinium Zwitterion

$\mathrm{N}-0.6282100 .047660-0.482180$

C $-0.1383600 .024890-1.739360$

C $0.7729200 .263190-4.374390$

C $1.274530-0.047290-2.006160$

C - $1.0797200 .168130-2.802920$

C -0.623330 0.278570 -4.091220

C $1.6894800 .110080-3.361700$

H $2.104830-0.6818501 .185250$

H -2.138300 $0.193920-2.561870$

H -1.330910 0.387320 -4.908960

H $2.7575400 .074290-3.554840$

H $1.1048400 .367270-5.404010$

N $0.251610-0.1357300 .485050$

C $1.591570-0.3848000 .273250$

C $2.219480-0.282200-0.964210$

C -0.292390 -0.061890 1.819170

C - $1.557500-0.5984102 .073690$

C -2.068120 -0.552180 3.368400

C - 1.3252300 .0249304 .401930

C -0.0672200 .5656204 .134230$

C 0.4554200 .5282802 .841230

H -2.119860 -1.040600 1.259910

H -3.048800 -0.973010 3.570910

$\mathrm{H}-1.7285600 .0574705 .409930$

H 0.5088801 .0307404 .928790

H 1.4174200 .9794002 .623120 


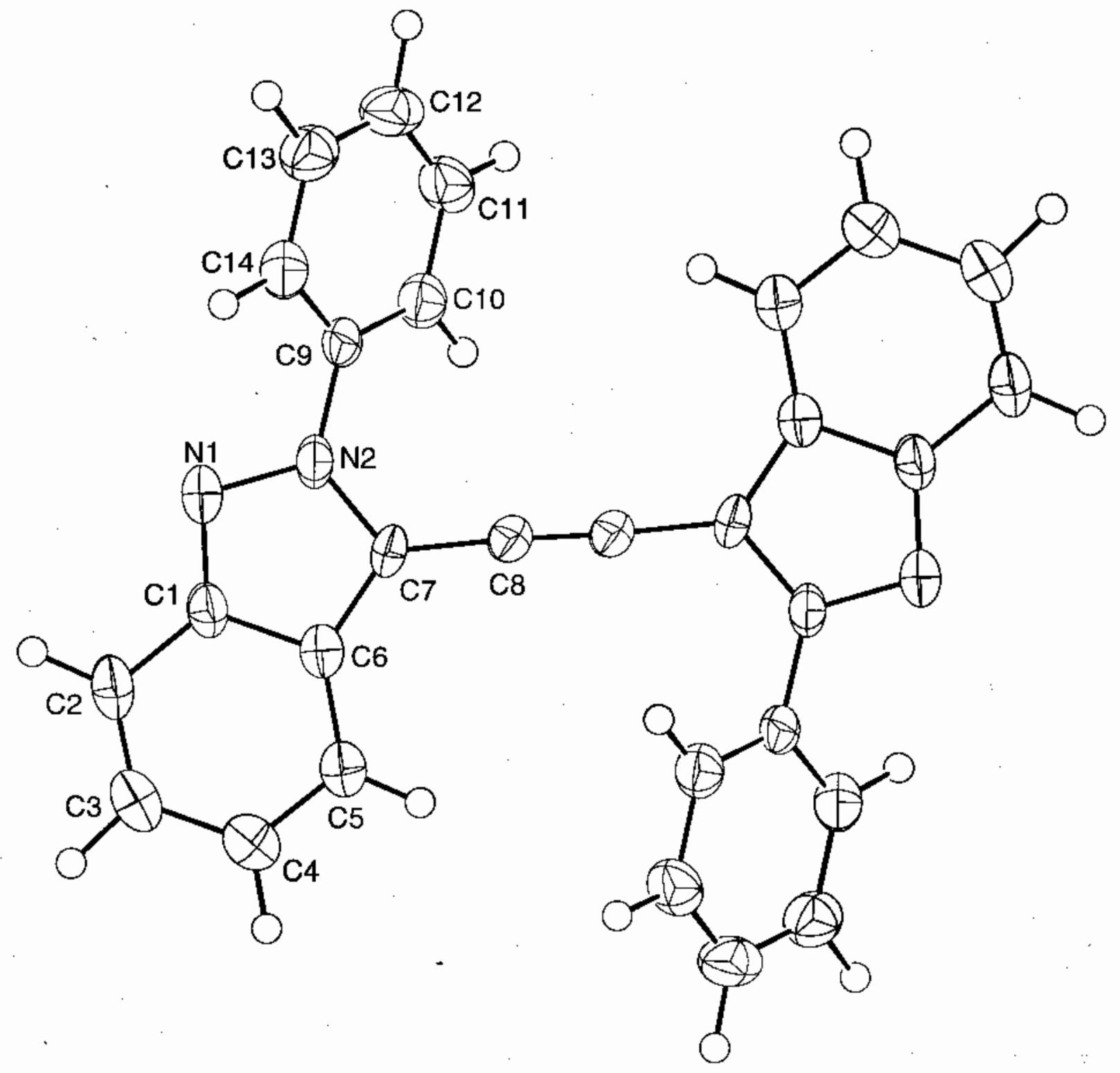




\section{crystal structural analysis}

Crystals were used as supplied. A needle of dimensions $0.044 \times 0.08 \times 0.36 \mathrm{~mm}$ was mounted in a fiber loop under oil and transferred to a Bruker SMART APEX diffractometer fitted with a CCD area detector. A total of 240030 -second frames were collected at $100(2) \mathrm{K}$ with a scan width of $0.3^{\circ}$ on $\omega$ or $\phi$ (software: $S M A R T^{1}$ ). The cell dimensions were refined from the coordinates of 4231 reflections in the range $5.1^{\circ} \leq \theta \leq 65.9^{\circ}$ (software: SAINT'). The following table contains a summary of crystal data and the final residuals. A more extensive table including particulars of data collection and structure refinement is in the supplementary material. The systematic absences and $2 / \mathrm{m}$ Lave symmetry indicated the space-group $\mathrm{P2}_{1} / c$. Absorption corrections were not required. A SIR92 E-map ${ }^{3}$ showed all non-hydrogen atoms. The final. difference synthesis was featureless. The TEXSAN program suite ${ }^{4}$ was used in all calculations.

\section{References}

1. Bruker AXS Inc., Madison, Wisconsin, USA. SMART software for data collection, 2001 .

2. Bruker AXS Inc., Madison, Wisconsin, USA. SAINT software for cell determination and data reduction, 2001.

3. Altomare, A.; Cascarano, G.; Giacovazzo, C.; Guagliardi, A.; Burla, M.C. ; Polidori. G.; Camalli, N. J. Appl. Crystallography, 1994, 27, 435 .

4. Molecular structures Corporation, 3200A Research Forest Drive, The Woodlands, TX 77381, USA. TeXsan Software for single-Crystal structure Analysis, version $1.7,1997$. 
Crystallographic data

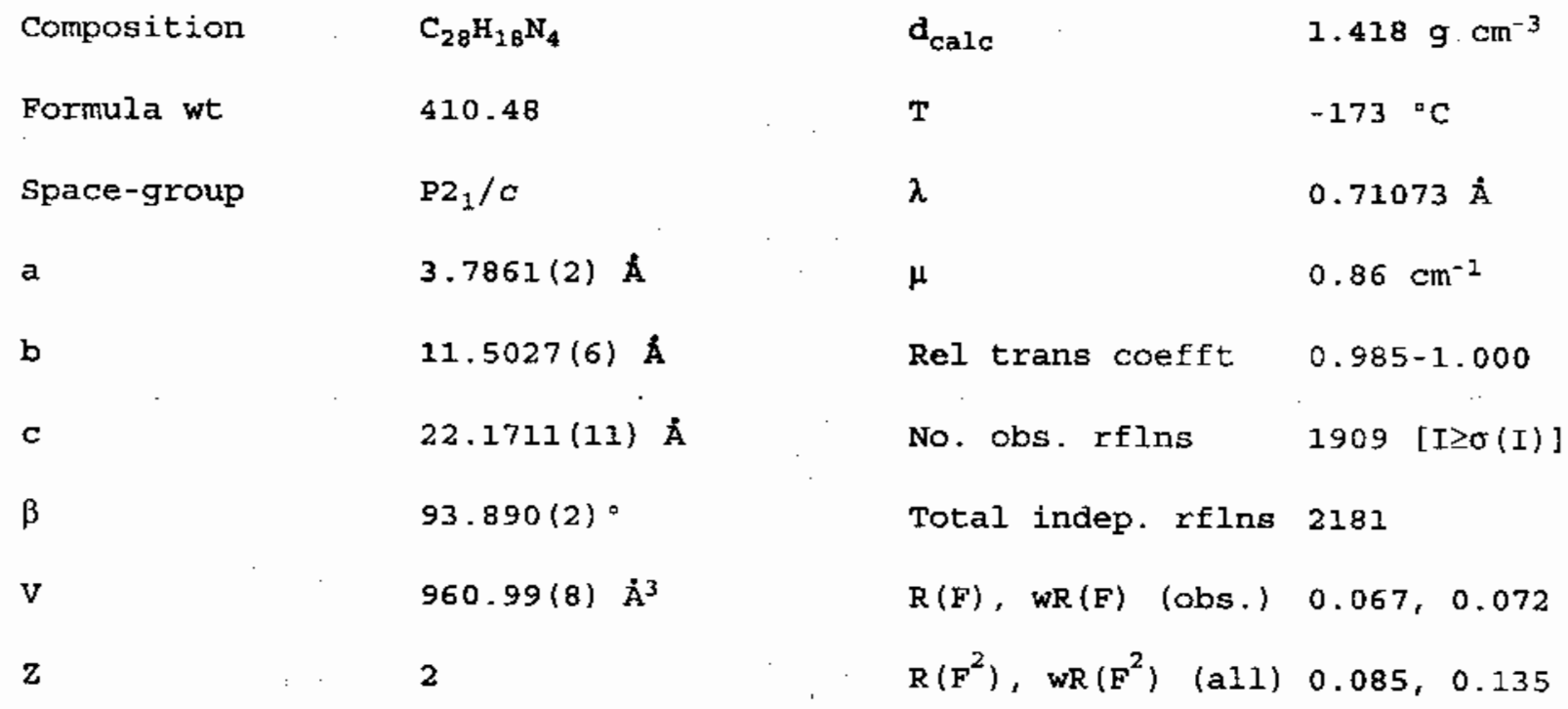

$$
R(F)=\Sigma|| F_{o}|-| F_{c}|| / \Sigma\left|F_{o}\right| \quad W R\left(F^{2}\right)=\left[\Sigma W\left(\left|F_{o}\right|^{2}-\left|F_{c}\right|^{2}\right)^{2} / \Sigma w\left|F_{o}\right|^{4}\right]^{3 / 5}
$$


Atomic coordinates and equivalent isotropic thermal parameters $\left(\dot{\mathrm{A}}^{2}\right)$

$$
B_{e q}=\left(8 \pi^{2} / 3\right) \Sigma_{i} \Sigma_{j} U_{i j} a^{*}{ }_{i} a^{*} a_{i} \cdot a_{j}
$$

$\begin{array}{lcccc}\text { Atom } & \mathbf{x} & \mathrm{y} & \mathrm{z} & \mathrm{B} \text { eq } \\ \mathrm{N}(1) & 0.8762(4) & 0.05678(13) & 0.32830(6) & 1.27(3) \\ \mathrm{N}(2) & 0.7533(4) & 0.08497(13) & 0.38256(6) & 1.17(3) \\ \mathrm{C}(1) & 0.8935(5) & -0.05895(16) & 0.32952(8) & 1.18(4) \\ \mathrm{C}(2) & 1.0136(5) & -0.13351(17) & 0.28446(8) & 1.37(4) \\ \mathrm{C}(3) & 1.0086(5) & -0.24921(17) & 0.29453(8) & 1.47(4) \\ \mathrm{C}(4) & 0.8877(5) & -0.29627(16) & 0.34883(8) & 1.41(4) \\ \mathrm{C}(5) & 0.7729(5) & -0.22638(16) & 0.39334(8) & 1.25(4) \\ \mathrm{C}(6) & 0.7779(4) & -0.10618(15) & 0.38404(8) & 1.09(3) \\ \mathrm{C}(7) & 0.6890(4) & -0.00907(15) & 0.41752(8) & 1.10(3) \\ \mathrm{C}(8) & 0.5550(5) & -0.00122(14) & 0.47527(8) & 1.10(3) \\ \mathrm{C}(9) & 0.7017(4) & 0.20376(15) & 0.39665(8) & 1.16(4) \\ \mathrm{C}(10) & 0.8201(5) & 0.24518(16) & 0.45294(8) & 1.30(4) \\ \mathrm{C}(11) & 0.7704(5) & 0.36065(16) & 0.46622(9) & 1.57(4) \\ \mathrm{C}(12) & 0.6086(5) & 0.43419(17) & 0.42316(9) & 1.58(4) \\ \mathrm{C}(13) & 0.4951(5) & 0.39147(16) & 0.36708(9) & 1.55(4) \\ \mathrm{C}(14) & 0.5399(5) & 0.27599(16) & 0.35332(8) & 1.35(4) \\ \mathrm{H}(1) & 1.095(5) & -0.1011(16) & 0.2476(9) & 1.3(4) \\ \mathrm{H}(2) & 1.088(5) & -0.3024(16) & 0.2650(9) & 0.9(4) \\ \mathrm{H}(3) & 0.887(5) & -0.3773(17) & 0.3541(8) & 1.0(4) \\ \mathrm{H}(4) & 0.676(6) & -0.2636(19) & 0.4283(10) & 2.3(5) \\ \mathrm{H}(5) & 0.930(5) & 0.1941(18) & 0.4828(10) & 2.0(4) \\ \mathrm{H}(6) & 0.866(6) & 0.3932(17) & 0.5047(10) & 2.0(4) \\ \mathrm{H}(7) & 0.586(5) & 0.5143(15) & 0.4310(8) & 0.5(3) \\ \mathrm{H}(8) & 0.376(5) & 0.4448(19) & 0.3360(9) & 1.8(4) \\ \mathrm{H}(9) & 0.454(5) & 0.2451(16) & 0.3149(9) & 1.5(4)\end{array}$


Bond lengths (A)

$\begin{array}{llll}N(1)-N(2) & 1.356(2) & C(6)-C(7) & 1.394(2) \\ N(1)-C(1) & 1.333(2) & C(7)-C(8) & 1.409(2) \\ N(2)-C(7) & 1.361(2) & C(8)-C\left(8^{i}\right) & 1.197(3) \\ N(2)-C(9) & 1.418(2) & C(9)-C(10) & 1.379(3) \\ C(1)-C(2) & 1.413(2) & C(9)-C(14) & 1.380(3) \\ C(1)-C(6) & 1.419(2) & C(10)-C(11) & 1.376(3) \\ C(2)-C(3) & 1.350(3) & C(11)-C(12) & 1.385(3) \\ C(3)-C(4) & 1.421(3) & C(12)-C(13) & 1.375(3) \\ C(4)-C(5) & 1.365(2) & C(13)-C(14) & 1.376(3) \\ C(5)-C(6) & 1.398(3) & & \end{array}$

(i) $1-x, y, 1-z$ 


\section{Bond angles $\left({ }^{\circ}\right)$}

\begin{tabular}{|c|c|c|c|}
\hline$N(2)-N(1)-C(1)$ & $103.9(1)$ & $c(5)-c(6)-c(7)$ & $135.1(2)$ \\
\hline$N(1)-N(2)-C(7)$ & $113.5(1)$ & $\mathrm{N}(2)-\mathrm{C}(7)-\mathrm{C}(6)$ & $106.0(1)$ \\
\hline$N(1)-N(2)-C(9)$ & $119.1(1)$ & $N(2)-C(7)-C(8)$ & $123.6(2)$ \\
\hline$C(7)-N(2)-C(9)$ & $127.5(1)$ & $C(6)-C(7)-C(8)$ & $130.4\langle 2\rangle$ \\
\hline $\mathrm{N}(1)-\mathrm{C}(1)-\mathrm{C}(2)$ & $127.6(2)$ & $C(7)-C(8)-C\left(8^{i}\right)$ & $177.5(2)$ \\
\hline$N(1)-C(1)-C(6)$ & $112.5(2)$ & $N(2)-C(9)-C(10)$ & $119.3(2)$ \\
\hline$C(2)-C(1)-C(6)$ & $120.0(2)$ & $\mathrm{N}(2)-\mathrm{C}(9)-\mathrm{C}(14)$ & $119.3(2)$ \\
\hline$C(1)-C(2)-C(3)$ & $118.2(2)$ & $C(10)-C(9)-C(14)$ & $121.4(2)$ \\
\hline$C(2)-C(3)-C(4)$ & $121.6(2)$ & $c(9)-c(10)-C(11)$ & $119.0(2)$ \\
\hline$C(3)-C(4)-C(5)$ & $121.5(2)$ & $C(10)-C(11)-C(12)$ & $120.2(2)$ \\
\hline$C(4)-C(5)-C(6)$ & $117.9(2)$ & $C(11)-C(12)-C(13)$ & $119.9(2)$ \\
\hline$C(1)-C(6)-C(5)$ & $120.8(2)$ & $C(12)-C(13)-C(14)$ & $120.6(2)$ \\
\hline$c(1)-c(6)-c(7)$ & $104.1(2)$ & $C(9)-C(14)-C(13)$ & $118.9(2)$ \\
\hline (i) $1-x,-y$, & & & \\
\hline
\end{tabular}




\section{Crystal data}

Formula

Formula weight

Crystal system, space-group

$a, b, c$

$\alpha, \beta, \gamma$

V

z

$\mathrm{d}_{\text {calc }}$

$\mu$

$\mathrm{F}_{000}$

\section{Data collection}

Crystal appearance

Crystal dimensions

Diffractometer

Radiation, wavelength

Monochromator

Temperature

$2 \theta_{\max }$

Index range $h, k, l$

Frames: number, time, w width

No. rflns scanned, $R_{\text {int }}$

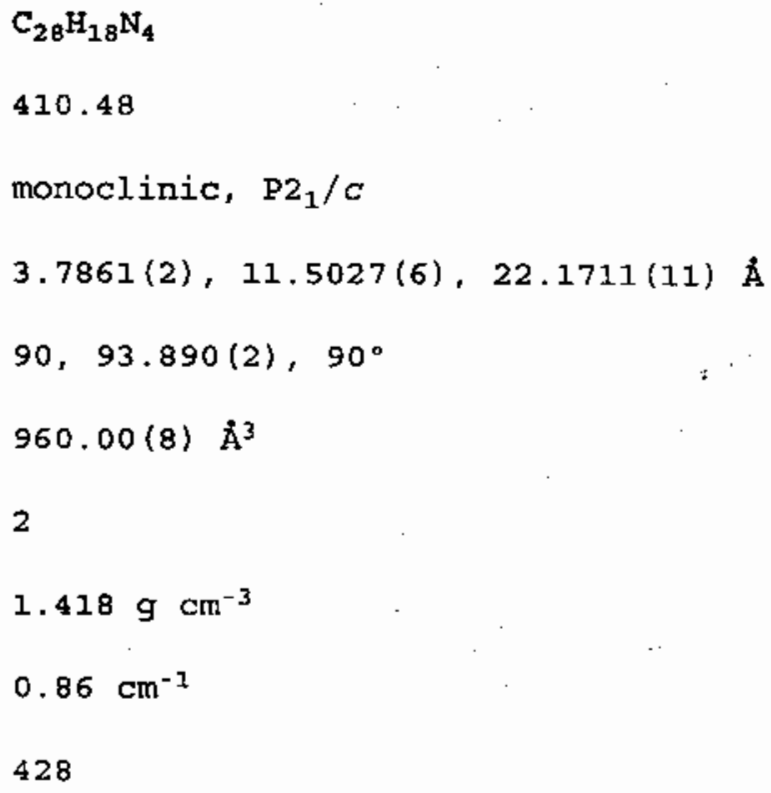




\section{Refinement}

Absorption correction

Sec. extinction parameter

No, rflns in refinement (N)

No. parameters (V)

Refinement on

Weighting factor w

$R(F), \quad W R(F) \quad[I \geq \sigma(I)]$

$R\left(F^{2}\right)$ ， WR $\left(F^{2}\right)$ (all data)

$S[I \geq \sigma(I)], S$ (all data)

Max. $\Delta / \sigma$, last cycle

Max., min. in final diff. map

\section{none}

predicted neg., fixed at zero

$1909[I \geq \sigma(I)]$.

2181 (all indep. data)

181

$|F|^{2}$

$1 / \sigma^{2}(\mathrm{~F})$

$0.067,0.072$

$0.085,0.135$

$1.46,1.33$

0.01

$0.41,-0.31$ e $\AA^{-3}$

$$
\begin{gathered}
R(F)=\Sigma|| F_{o}|-| F_{c}|| / \Sigma\left|F_{o}\right| \quad W R\left(F^{2}\right)=\left[\Sigma W\left(\left|F_{o}\right|^{2}-\left|F_{c}\right|^{2}\right)^{2} / \Sigma W\left|F_{o}\right|^{4}\right]^{k /} \\
S=\left[\Sigma W\left(\left|F_{o}\right|-\left|F_{c}\right|\right)^{2} /(N-V)\right]^{\not / 2}
\end{gathered}
$$


Anisotropic thermal parameters $\left(\AA^{2}\right)$

The temperature factor is given by: $T=\exp \left[-2 \pi^{2} \Sigma_{i} \Sigma_{j} U_{i j} h_{i} h_{j} a{ }_{i} a{ }_{j}\right]$

$\begin{array}{llllccc}\text { Atom } & \mathrm{U}_{11} & \mathrm{U}_{22} & \mathrm{U}_{33} & \mathrm{U}_{12} & \mathrm{U}_{13} & \mathrm{U}_{23} \\ \mathrm{~N}(1) & 0.0204(8) & 0.0206(8) & 0.0080(8) & -0.0001(6) & 0.0067(6) & -0.0021(6) \\ \mathrm{N}(2) & 0.0199(8) & 0.0179(8) & 0.0073(7) & 0.0002(6) & 0.0060(6) & 0.0001(6) \\ \mathrm{C}(1) & 0.0157(9) & 0.0199(10) & 0.0093(9) & -0.0013(7) & 0.0015(7) & 0.0015(7) \\ \mathrm{C}(2) & 0.0185(9) & 0.0256(10) & 0.0086(9) & -0.0001(7) & 0.0042(7) & -0.0014(7) \\ \mathrm{C}(3) & 0.0188(9) & 0.0246(10) & 0.0127(9) & 0.0034(7) & 0.0034(7) & -0.0070(8) \\ \mathrm{C}(4) & 0.0222(10) & 0.0149(10) & 0.0166(10) & 0.0004(7) & 0.0019(7) & -0.0023(7) \\ \mathrm{C}(5) & 0.0167(9) & 0.0193(10) & 0.0118(9) & -0.0010(7) & 0.0022(7) & -0.0003(7) \\ \mathrm{C}(6) & 0.0123(8) & 0.0211(10) & 0.0083(8) & -0.0001(6) & 0.0021(6) & -0.0018(7) \\ \mathrm{C}(7) & 0.0147(8) & 0.0188(10) & 0.0086(8) & -0.0018(6) & 0.0028(6) & 0.0002(7) \\ \mathrm{C}(8) & 0.0167(8) & 0.0139(9) & 0.0114(9) & 0.0003(6) & 0.0025(6) & -0.0002(7) \\ \mathrm{C}(9) & 0.0162(9) & 0.0159(9) & 0.0128(9) & -0.0011(6) & 0.0082(7) & -0.0002(7) \\ C(10) & 0.0172(9) & 0.0209(10) & 0.0116(9) & -0.0014(7) & 0.0044(7) & 0.0015(8) \\ C(11) & 0.0232(9) & 0.0209(10) & 0.0165(10) & -0.0041(7) & 0.0075(8) & -0.0032(8) \\ C(12) & 0.0222(10) & 0.0159(10) & 0.0232(10) & 0.0001(7) & 0.0103(8) & -0.0017(8) \\ C(13) & 0.0213(9) & 0.0204(10) & 0.0177(10) & 0.0016(7) & 0.0062(8) & 0.0040(8) \\ C(14) & 0.0190(9) & 0.0224(10) & 0.0105(9) & -0.0010(7) & 0.0053(7) & 0.0012(7)\end{array}$




\section{Supporting data}

Bond lengths (Ả) for hydrogen atoms

$\begin{array}{llllll}\mathrm{C}(2) & \mathrm{H}(1) & 0.97(2) & \mathrm{C}(11) & \mathrm{H}(6) & 0.98(2) \\ \mathrm{C}(3) & \mathrm{H}(2) & 0.96(2) & \mathrm{C}(12) & \mathrm{H}(7) & 0.94(2) \\ \mathrm{C}(4) & \mathrm{H}(3) & 0.94(2) & \mathrm{C}(13) & \mathrm{H}(8) & 1.00(2) \\ \mathrm{C}(5) & \mathrm{H}(4) & 0.98(2) & \mathrm{C}(14) & \mathrm{H}(9) & 0.96(2) \\ \mathrm{C}(10) & \mathrm{H}(5) & 0.96(2) & & & \end{array}$


Intermolecular distances ( $\dot{A})$ for non-hydrogen atoms

$\begin{array}{llllll}\mathrm{N}(1) & \mathrm{N}\left(2^{i}\right) & 3.469(2) & \mathrm{C}(3) & \mathrm{C}\left(5^{1}\right) & 3.516(3) \\ \mathrm{N}(1) & \mathrm{C}\left(3^{\mathrm{i}}\right) & 3.565(2) & \mathrm{C}(4) & \mathrm{C}\left(5^{i}\right) & 3.522(3) \\ \mathrm{N}(1) & \mathrm{C}\left(14^{\mathrm{i}}\right) & 3.576(2) & \mathrm{C}(7) & \mathrm{C}\left(8^{i}\right) & 3.438(3) \\ \mathrm{C}(1) & \mathrm{C}\left(2^{\mathrm{i} i}\right) & 3.518(3) & \mathrm{C}(7) & \mathrm{C}\left(8^{i v}\right) & 3.593(3) \\ \mathrm{C}(1) & \mathrm{C}\left(7^{i}\right) & 3.521(2) & \mathrm{C}(8) & \mathrm{C}\left(8^{i v}\right) & 3.472(3) \\ \mathrm{C}(1) & \mathrm{C}\left(6^{i}\right) & 3.523(2) & \mathrm{C}(9) & \mathrm{C}\left(14^{\mathrm{i}}\right) & 3.477(2) \\ \mathrm{C}(2) & \mathrm{C}\left(6^{i}\right) & 3.530(3) & \mathrm{C}(11) & \mathrm{C}\left(12^{\mathrm{i}}\right) & 3.479(2) \\ \mathrm{C}(3) & \mathrm{C}\left(4^{i}\right) & 3.503(3) & & & \end{array}$

Symmetry code:

(i) $1+x, y, z$; (ii) $2-x, 0.5+y, 0.5-z$; (iii) $-1+x, y, z$;

(iv) $2-x, 1-y,-z$ 


\begin{tabular}{|c|c|c|c|c|c|c|c|c|c|}
\hline (1) & (2) & (3) & (4) & angle & (1) & (2) & (3) & (4) & angle \\
\hline $\mathbf{N}(1)$ & $N(2)$ & $C(7)$ & $C(6)$ & $0.4(2)$ & $C(2)$ & $C(1)$ & $C(6)$ & $C(5)$ & $-1.6(3)$ \\
\hline $\mathrm{N}(1)$ & $\mathbf{N}(2)$ & $C(7)$ & $C(8)$ & $-178.9(2)$ & $C(2)$ & $C(1)$ & $C(6)$ & $C(7)$ & $178.6(2)$ \\
\hline $\mathrm{N}(1)$ & $\mathbf{N}(2)$ & $C(9)$ & $C(10)$ & $-134.5(2)$ & $C(2)$ & $C(3)$ & $C(4)$ & $C(5)$ & $-0.4(3)$ \\
\hline$N(1)$ & $N\langle 2\rangle$ & $C(9)$ & $C(14)$ & $44.3(2)$ & $C(3)$ & $C(2)$ & $C(1)$ & $C(6)$ & $1.1(3)$ \\
\hline$N(1)$ & $C(1)$ & $C(2)$ & $c(3)$ & $-179.8(2)$ & $C(3)$ & $C(4)$ & $C(5)$ & $C(6)$ & $-0.1(3)$ \\
\hline$N(1)$ & $C(1)$ & $C(6)$ & $C(5)$ & $179.2(1)$ & $C(4)$ & $C(5)$ & $C(6)$ & $c(7)$ & $-179.3(2)$ \\
\hline$N(1)$ & $C(1)$ & $C(6)$ & $C(7)$ & $-0.6(2)$ & $c(5)$ & $C(6)$ & $C(7)$ & $C(8)$ & $-0.4(3)$ \\
\hline $\mathrm{N}(2)$ & $\mathbf{N}(1)$ & $C(1)$ & $C(2)$ & $-178.4(2)$ & $c(6)$ & $C(7)$ & $\mathbf{N}(2)$ & $C(9)$ & $179.5(2)$ \\
\hline $\mathbf{N}(2)$ & $\mathrm{N}(1)$ & $C(1)$ & $c(6)$ & $0.8(2)$ & $c(6)$ & $C(7)$ & $C(8)$ & $C(8)$ & $17(6)$ \\
\hline $\mathbf{N}(2)$ & $c(7)$ & $C(6)$ & $C(1)$ & $0.1(2)$ & $C(7)$ & $\mathbf{N}(2)$ & $C(9)$ & $C(10)$ & $46.4(2)$ \\
\hline$N(2)$ & $c(7)$ & $c(6)$ & $c(5)$ & $-179.7(2)$ & $C(7)$ & $\mathbf{N}(2)$ & $C(9)$ & $C(14)$ & $-134.8(2)$ \\
\hline$N(2)$ & $C(7)$ & $C(8)$ & $C(B)$ & $-164(6)$ & $C(7)$ & $C(8)$ & $C(8)$ & $C(7)$ & 180 \\
\hline$N(2)$ & $c(9)$ & $C(10)$ & $\mathrm{C}(11)$ & $179.7(1)$ & $C(8)$ & $c(7)$ & $\mathrm{N}(2)$ & $C(9)$ & $0.2(3)$ \\
\hline $\mathbf{N}(2)$ & $C(9)$ & $C(14)$ & $C(13)$ & $-179.1(1)$ & $c(9)$ & $C(10)$ & $C(11)$ & $\mathrm{C}(12)$ & $-1.0(2)$ \\
\hline$C(1)$ & $\mathbf{N}(1)$ & $N(2)$ & $C(7)$ & $-0.7(2)$ & $c(9)$ & $C(14)$ & $\mathrm{C}(13)$ & $c(12)$ & $-0.2(2)$ \\
\hline$C(1)$ & $\mathrm{N}(1)$ & $\mathbf{N}(2)$ & $c(9)$ & $-179.9(1)$ & $C(10)$ & $C(9)$ & $C(14)$ & $C(13)$ & $-0.4(2)$ \\
\hline$C(1)$ & $C(2)$ & $C(3)$ & $C(4)$ & $\begin{array}{l}-0.2(3) \\
.\end{array}$ & $C(10)$ & $C(11)$ & $\mathrm{C}(12)$ & $\mathrm{C}(1.3)$ & $0.4(2)$ \\
\hline$C(1)$ & $c(6)$ & $C(5)$ & $C(4)$ & $1.0(3)$ & $C(11)$ & $C(10)$ & $C(9)$ & $C(14)$ & $1.0(2)$ \\
\hline$C(1)$ & $C(6)$ & $C(7)$ & $C(8)$ & $179.4(2)$ & $C(11)$ & $C(12)$ & $C(13)$ & $C(14)$ & $0.2(3)$ \\
\hline
\end{tabular}




\section{Supporting data}

Least-squares mean planes (deviations in $\dot{A}$ )

Plane...

\begin{tabular}{cr} 
Defining atoms & \multicolumn{1}{c}{ Distance } \\
$N(1)$ & $-0.014(2)$ \\
$N(2)$ & $0.007(2)$ \\
$C(1)$ & $-0.007(2)$ \\
$C(2)$ & $0.010(2)$ \\
$C(3)$ & $0.010(2)$ \\
$C(4)$ & $-0.004(2)$ \\
$C(5)$ & $-0.010(2)$ \\
$C(6)$ & $-0.001(2)$ \\
$C(7)$ & $0.010(2)$
\end{tabular}

Mean deviation from plane: $0.008 \dot{A}_{i} \chi^{2}, 272$

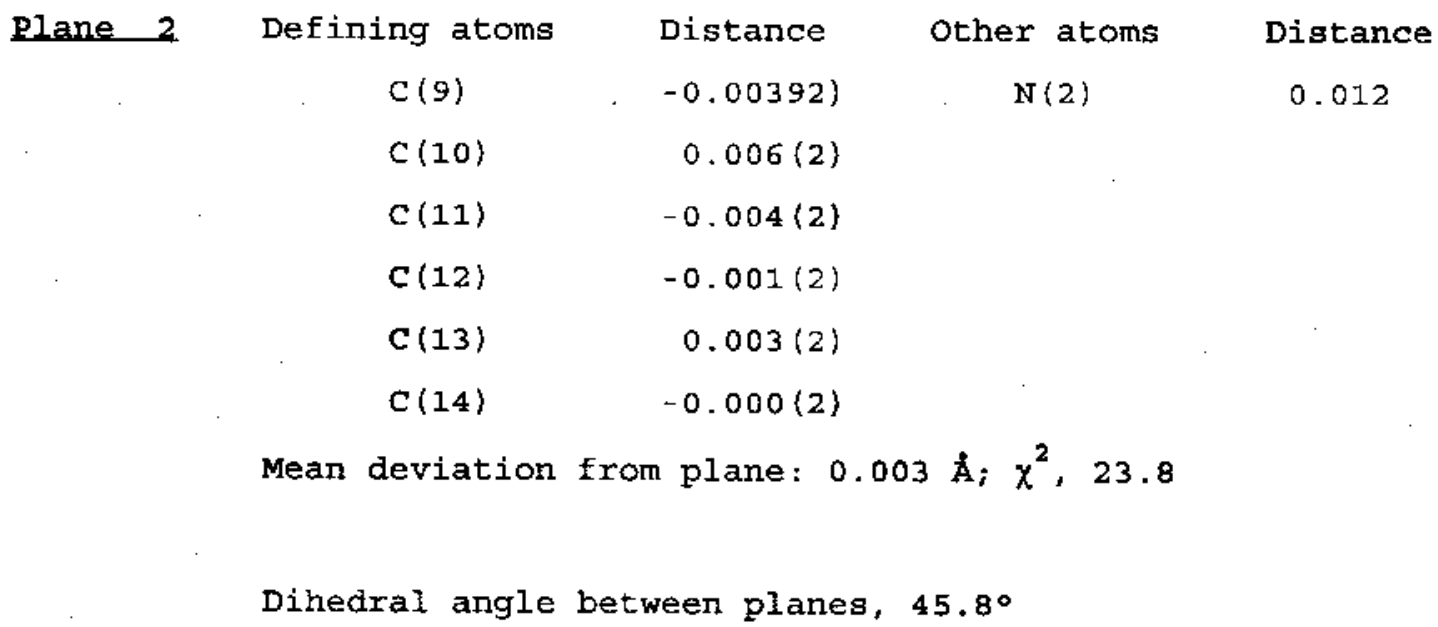




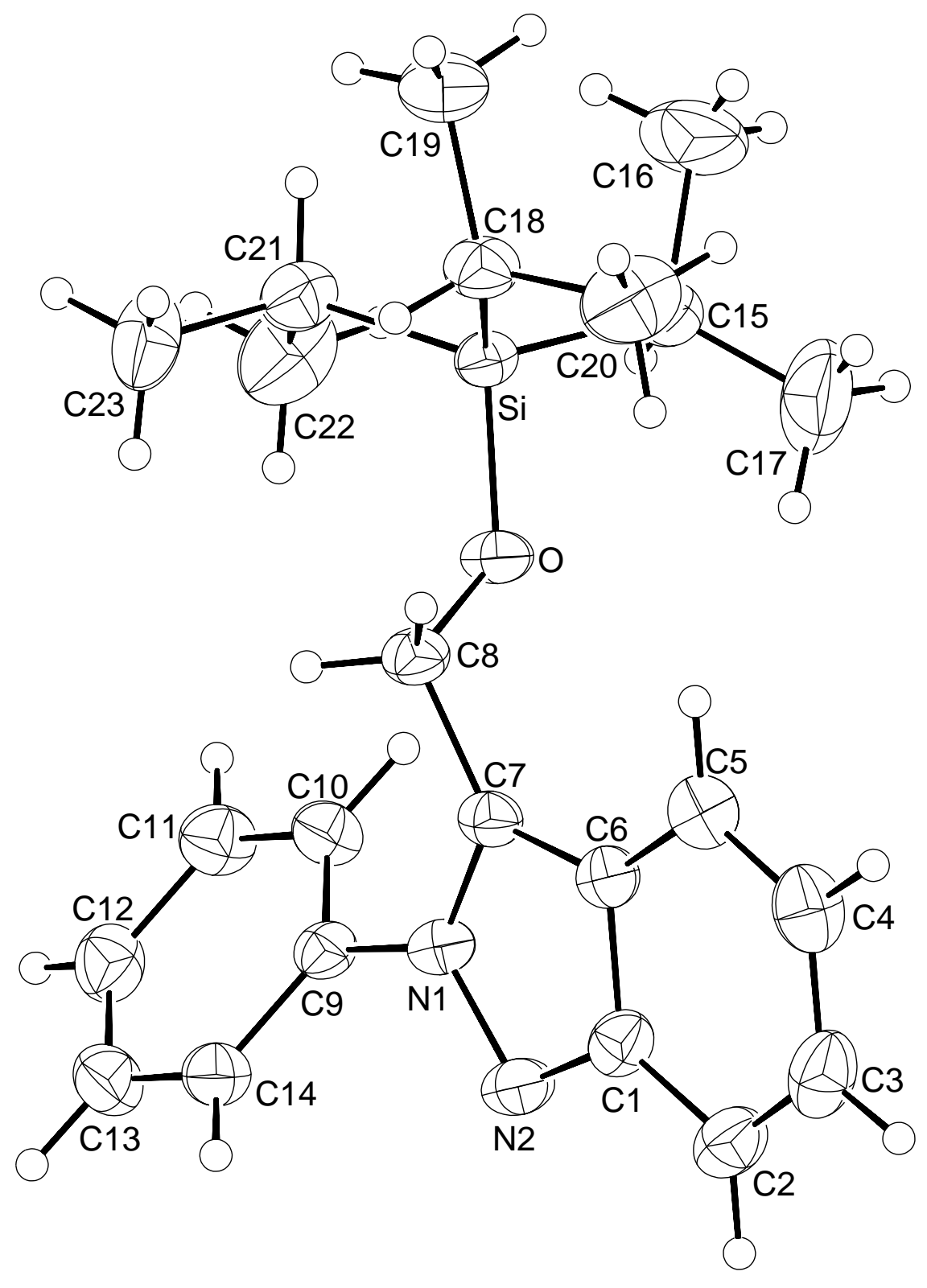




\section{Crystal structural analysis}

A crystal of dimensions $0.02 \times 0.08 \times 0.50 \mathrm{~mm}$ was mounted on a fiber. The orientation parameters and cell dimensions were obtained from the setting angles of a Nonius CAD-4 serial diffractometer for 25 centered reflections in the range $12.2^{\circ} \leq \theta \leq 13.5^{\circ}$. The following table contains a summary of crystal data and the final residuals. A more extensive table including particulars of data collection and structure refinement is in the supplementary material. The monoclinic Laue symmetry and systematic absences together indicated the space-group $\mathrm{P2} / \mathrm{l}$. A SIR92 $\mathrm{E}$-map ${ }^{1}$ showed all the non-hydrogen atoms. Absorption and decay corrections were not deemed necessary. Hydrogen atoms were included at positions recalculated after each cycle of refinement $[B(H)=$ $\left.1.2 \mathrm{~B}_{\text {eg }}(\mathrm{C}) ; \mathrm{d}(\mathrm{C}-\mathrm{H})=0.95 \mathrm{~A}\right]$. The final difference synthesis was featureless. The TEXSAN program suite ${ }^{2}$ was used in all calculations.

\section{References}

1. Altomare, A.; Cascarano, G.; Giacovazzo, C.; Guagliardi, A.; Burla, M.C.; Polidori, G.; Camalli, N. J. App1. Crystallography, 1994, 27, 435 .

2. Molecular Structures Corporation, 3200A Research Forest Drive, The Woodlands, TX 77381, USA. TeXsan Software for Single-Crystal structure Analysis, version $1.7,1997$. 


\section{Crystallographic data}

\begin{tabular}{|c|c|c|c|}
\hline Composition & $\mathrm{C}_{23} \mathrm{H}_{32} \mathrm{~N}_{2} \mathrm{OSi}$ & $d_{\text {calc }}$ & $1.116 \mathrm{~g} \mathrm{~cm}^{-3}$ \\
\hline Formula wt & 380.6 & $\mathrm{~T}$ & $23^{\circ} \mathrm{C}$ \\
\hline Space-group & $\mathrm{P} 2_{1} / \mathrm{C}$ & $\lambda$ & $0.71073 \dot{A}$ \\
\hline$a$ & $15.954(4) \AA$ & $\mu$ & $1.18 \mathrm{~cm}^{-1}$ \\
\hline b & $8.689\langle 3\rangle \dot{A}$ & No. obs. rflns & $2342[I \geq \sigma(I)]$ \\
\hline$c$ & $17.933(8)$ A & Total indep. rflns & 3925 \\
\hline$\beta$ & $114.35(3)^{\circ}$ & $R(F)$, wR (F) (obs.) & $0.063,0.057$ \\
\hline $\mathrm{v}$ & $2265(2) \dot{A}^{3}$ & $R\left(F^{2}\right), w R\left(F^{2}\right) \quad(a l l)$ & $0.104,0.120$ \\
\hline $\mathrm{z}$ & 4 & & \\
\hline
\end{tabular}

$$
R(F)=\Sigma|| F_{0}|-| F_{c}|| / \Sigma\left|F_{0}\right| \quad W R\left(F^{2}\right)=\left[\Sigma W\left(\left|F_{o}\right|^{2}-\left|F_{C}\right|^{2}\right)^{2} / \Sigma W\left|F_{o}\right|^{4}\right]^{1 / 2}
$$


Atomic coordinates and equivalent isotropic thermal parameters $\left(\dot{A}^{2}\right)$

$$
B_{e q}=\left(8 \pi^{2} / 3\right) \Sigma_{i} \Sigma_{j} U_{i j} a^{*}{ }_{i}{ }^{*}{ }_{j} a_{i} \cdot a_{j}
$$

\begin{tabular}{|c|c|c|c|c|}
\hline Atom & $\mathbf{x}$ & $y$ & $z$ & $B_{\text {eq }}$ \\
\hline $\mathrm{si}$ & $0.31838(6)$ & $0.76234(10)$ & $0.16218(5)$ & $3.726(19)$ \\
\hline 0 & $0.25820(12)$ & $0.6035(2)$ & $0.12509(11)$ & $4.01(5)$ \\
\hline$N(1)$ & $0.13294(16)$ & $0.3160\langle 3\}$ & $0.01636(14)$ & $3.84\langle 6\rangle$ \\
\hline$N(2)$ & $0.08227(17)$ & $0.1924(3)$ & $0.02048(16)$ & $4.51(7)$ \\
\hline$c(1)$ & $0.04329(19)$ & $0.2415\{4\rangle$ & $0.07026(18)$ & $4.16(8)$ \\
\hline$c(2)$ & $-0.0164(2)$ & $0.1585\{4\rangle$ & $0.0953(2)$ & $5.24(9)$ \\
\hline$C(3)$ & $-0.0483(2)$ & $0.2302(5)$ & $0.1453(2)$ & $5.92(11)$ \\
\hline$C(4)$ & $-0.0248(2)$ & $0.3855(5)$ & $0.1708\{2\rangle$ & $5.71(10)$ \\
\hline$C(5)$ & $0.0327(2)$ & $0.4664(4)$ & $0.14705(19)$ & $4.77\langle 9\rangle$ \\
\hline$C(6)$ & $0.06882(18)$ & $0.3956(4)$ & $0.09664(17)$ & $3.75(7)$ \\
\hline$C(7)$ & $0.12715(18)$ & $0.4395(3)$ & $0.06052(17)$ & $3.56(7)$ \\
\hline$c(8)$ & $0.16916(19)$ & $0.5927(3)$ & $0.06029(17)$ & $3.96(8)$ \\
\hline$C(9)$ & $0.1866(2)$ & $0.3001(3)$ & $-0.03045(18)$ & $3.60(7)$ \\
\hline$c(10)$ & $0.2764(2)$ & $0.3489(4)$ & $0.00163(18)$ & $4.54(8)$ \\
\hline$C(11)$ & $0.3280(2)$ & $0.3295(4)$ & $-0.0431(2)$ & $5.21(9)$ \\
\hline$C(12)$ & $0.2907(2)$ & $0.2600(4)$ & $-0.1182(2)$ & $5.34(9)$ \\
\hline$C(13)$ & $0.2008(3)$ & $0.2114(4)$ & $-0.25000(18)$ & $5.36(10)$ \\
\hline$C(14)$ & $0.1482(2)$ & $0.2308(4)$ & $-0.10618(18)$ & $4.62(8)$ \\
\hline$c(15)$ & $0.4077(2)$ & $0.6924(4)$ & $0.2623(2)$ & $5.60(9)$ \\
\hline$c(16)$ & $0.4753(3)$ & $0.8125\langle 5\rangle$ & $0.3137(3)$ & $10.91(15)$ \\
\hline$C(17)$ & $0.3723(3)$ & $0.5958(6)$ & $0.3114(3)$ & $10.48(16)$ \\
\hline$C(18)$ & $0.2403(2)$ & $0.9192(4)$ & $0.16709(19)$ & $4.74(9)$ \\
\hline$C(19)$ & $0.2860(3)$ & $1.0783(4)$ & $0.1869\{2\rangle$ & $6.46(21)$ \\
\hline$C(20)$ & $0.1917(3)$ & $0.8863(5)$ & $0.2232(3)$ & $8.10(13)$ \\
\hline$c(21)$ & $0.3745(2)$ & $0.8305(4)$ & $0.0950(2)$ & $5.23(9)$ \\
\hline$C(22)$ & $0.4444(3)$ & $0.7191(5)$ & $0.0878(3)$ & $8.52(14)$ \\
\hline$C(23)$ & $0.3064(3)$ & $0.8782(5)$ & $0.0103(2)$ & $7.69(13)$ \\
\hline
\end{tabular}




\section{Bond lengths (A)}

\begin{tabular}{|c|c|c|c|}
\hline si-o & $1.654(2)$ & $C(6)-C(7)$ & $1.387(4)$ \\
\hline$s i-c(15)$ & $1.871(3)$ & $C(7)-C(8)$ & $1.490(4)$ \\
\hline $\mathrm{Si}-\mathrm{C}(18)$ & $1.870(3)$ & $c(9)-c(10)$ & $1.370(4)$ \\
\hline$S i-C(21)$ & $1.868(3)$ & $C(9)-C(14)$ & $1.375(4)$ \\
\hline $0-C(8)$ & $1.418(3)$ & $c(10)-C(11)$ & $1.375(4)$ \\
\hline$N(1)-N(2)$ & $1.363\langle 3\rangle$ & $C(11)-C(12)$ & $1.366(4)$ \\
\hline $\mathrm{N}(1)-\mathrm{C}(7)$ & $1.358(3)$ & $C(12)-C(13)$ & $1.371(4)$ \\
\hline$N(1)-C(9)$ & $1.430(3)$ & $C(13)-C(14)$ & $1.374(4)$ \\
\hline$N(2)-C(1)$ & $1.349(4)$ & $C(15)-C(16)$ & $1.509(5)$ \\
\hline$C(1)-C(2)$ & $1.406(4)$ & $C(15)-C(17)$ & $1.485(5)$ \\
\hline$c(1)-c(6)$ & $1.421(4)$ & $C(18)-C(19)$ & $1.533(4)$ \\
\hline$C(2)-C(3)$ & $1.350(5)$ & $C(18)-C(20)$ & $1.527(4)$ \\
\hline$C(3)-C(4)$ & $1.423(5)$ & $C(21)-C(22)$ & $1.520(5)$ \\
\hline$C(4)-C(5)$ & $1.355(4)$ & $C(21)-C(23)$ & $1.515(5)$ \\
\hline$C(5)-C(6)$ & $1.397(4)$ & & \\
\hline
\end{tabular}




\section{Bond angles (०)}

\begin{tabular}{|c|c|c|c|}
\hline $0-S i-C(15)$ & $101.5(1)$ & $N(1)-C(7)-C(6)$ & $105.9(3)$ \\
\hline$O-S i-C(18)$ & $109.8(1)$ & $N(1)-C(7)-C(8)$ & $124.4(3)$ \\
\hline$O-S i-C(21)$ & $111.0(1)$ & $C(6)-C(7)-C(8)$ & $129.4(3)$ \\
\hline$C(15)-S i-c(18)$ & $116.7(1)$ & $O-C(8)-C(7)$ & $110.8(2)$ \\
\hline$C(15)-S i-C(21)$ & $110.1(2)$ & $N(1)-C(9)-C(10)$ & $119.8(3)$ \\
\hline$C(18)-S i-C(21)$ & $107.7(2)$ & $N(1)-C(9)-C(14)$ & $119.3(3)$ \\
\hline $\mathrm{Si}-0-\mathrm{C}(8)$ & $127.1(2)$ & $C(10)-C(9)-C(14)$ & $120.8(3)$ \\
\hline$N(2)-N(1)-C(7)$ & $114.0(2)$ & $C(9)-C(10)-C(11)$ & $119.3(3)$ \\
\hline$N(2)-N(1)-C(9)$ & $117.8(2)$ & $C(10)-C(11)-C(12)$ & $120.4(3)$ \\
\hline$C(7)-N(1)-C(9)$ & $128.2(2)$ & $C(11)-C(12)-C(13)$ & $120.0(3)$ \\
\hline$N(1)-N(2)-C(I)$ & $103.4(2)$ & $C(12)-C(13)-C(14)$ & $120.2(3)$ \\
\hline$N(2)-C(1)-C(2)$ & $127.3(3)$ & $C(9)-C(14)-C(13)$ & $119.3(3)$ \\
\hline$N(2)-C(1)-C(6)$ & $111.8(3)$ & $S i-C(15)-C(16)$ & $115.5(3)$ \\
\hline$C(2)-C(1)-C(6)$ & $121.0(3)$ & $S i-C(15)-C(17)$ & $115.2(3)$ \\
\hline$C(1)-c(2)-c(3)$ & $117.7(3)$ & $C(16)-C(15)-C(17)$ & $112.2(3)$ \\
\hline$C(2)-C(3)-C(4)$ & $121.9(3)$ & $S i-C(18)-C(19)$ & $114.0(2)$ \\
\hline$C(3)-C(4)-C(5)$ & $120.9(3)$ & $S i-C(18)-C(20)$ & $115.0(2)$ \\
\hline$C(4)-C(5)-C(6)$ & $119.1(3)$ & $C(19)-C(18)-C(20)$ & $109.7(3)$ \\
\hline$C(1)-C(6)-C(5)$ & $119.5(3)$ & $S i-C(21)-C(22)$ & $114.5(3)$ \\
\hline$C(1)-C(6)-C(7)$ & $105.0(3)$ & $S 1-C(21)-C(23)$ & $113.3(2)$ \\
\hline$C(5)-C(6)-C(7)$ & $135.5(3)$ & $C(22)-C(21)-C(23)$ & $109.7(3)$ \\
\hline
\end{tabular}


Crystallographic information

\section{Crystal data}

Formula

$\mathrm{C}_{23} \mathrm{H}_{32} \mathrm{~N}_{2} \mathrm{OS} 1$

Formula weight

380.6

Crystal system, space-group

monoclinic, $\mathrm{P} 2{ }_{1} / c$
$a, b, c$
$\alpha, \beta, \gamma$
$\mathrm{v}$
$z$

$15.954(4), 8.689(3), 17.933(8) \dot{A}$

$90,114.35(3), 90^{\circ}$

$2265(2) \dot{A}^{3}$

4

$\mathrm{d}_{\text {calc }}$

$1.116 \mathrm{~g} \mathrm{~cm}^{-3}$

$\mu$

$1.18 \mathrm{~cm}^{+1}$

$F_{000}$

824

\section{Data collection}

Crystal appearance

Crystal dimensions

Diffractometer

Radiation, wavelength

Monochromator

Temperature

$2 \theta_{\max }$

Index range $h, k, I$

Scan mode, speed (on $\omega$ )

Scan width colorless prism

$0.02 \times 0.08 \times 0.50 \mathrm{~mm}$

Enraf-Nonius CAD-4

Mo $\mathrm{K \alpha}, 0.71073 \AA$

graphite

$23^{\circ} \mathrm{C}$

$50^{\circ}$

$0 \rightarrow 18, \quad 0 \rightarrow 10, \quad-21 \rightarrow 19$

$\omega-2 \theta, \quad 1.0-4.1^{\circ} \min ^{-1}$

$(1.05+0.35 \tan \theta)^{\circ}$ 
Reference reflections

No. indep. rflns scanned, $\mathbf{R}_{\text {int }}$

\section{Refinement}

Absorption correction

Sec. extinction parameter

No. rflns in refinement (N)

No. parameters (V)

Refinement on

Weighting factor w

$R\{F\}, w R(F) \quad[I \geq \sigma(I)]$

$R\left(F^{2}\right)$, wR $\left(F^{2}\right)$ (all data)

$s \quad[I \geq \sigma(I)], s$ (all data)

$\operatorname{Max}: \Delta / \sigma$, last cycle

Max., min. in final diff. map
3, every 3600 s exposure

$4292,0.038$

none

refined neg., fixed at zero

$2342[I \geq \sigma(I)]$

3925 (all indep. data)

244

$|\mathrm{F}|^{2}$

$1 / \sigma^{2}(F)$

$0.063,0.057$

$0.104,0.1 \div 20$

$1.64,1.37$

0.01

$0.41,-0.44$ e $\AA^{-3}$

$$
\begin{gathered}
R(F)=\Sigma|| F_{0}|-| F_{c}|| / \Sigma\left|F_{0}\right| \quad W R\left(F^{2}\right)=\left[\Sigma W\left(\left|F_{o}\right|^{2}-\left|F_{c}\right|^{2}\right)^{2} / \Sigma W\left|F_{o}\right|^{4}\right]^{3 / 2} \\
S=\left[\Sigma W\left(\left|F_{o}\right|-\left|F_{c}\right|\right)^{2} /(N-V)\right]^{3 / 2}
\end{gathered}
$$


Anisotxopic thermal parameters $\left(\AA^{2}\right)$

The temperature factor is given by: $T=\exp \left[-2 \pi^{2} \sum_{i} \Sigma_{j} U_{i j} h_{i} h_{j} a{ }_{i} a{ }_{j}\right]$

\begin{tabular}{|c|c|c|c|c|c|c|}
\hline Atom & $v_{11}$ & $\mathrm{U}_{22}$ & $\mathrm{U}_{33}$ & $\mathrm{U}_{12}$ & $\mathrm{U}_{13}$ & $\mathrm{U}_{23}$ \\
\hline $\mathrm{Si}$ & $0.0519(5)$ & $0.0379(5)$ & $0.0498\langle 5\rangle$ & $-0.0036(5)$ & $0.0190(4)$ & $-0.0014(5)$ \\
\hline 0 & $0.0459(11)$ & $0.0361(13)$ & $0.0580(13)$ & $-0.0034(10)$ & $0.0090(10)$ & $-0.0034(10)$ \\
\hline$N(1)$ & $0.0480(15)$ & $0.0401(16)$ & $0.0563(16)$ & $-0.0060(13)$ & $0.0198(13)$ & $-0.0030(13)$ \\
\hline $\mathrm{N}(2)$ & $0.0594(17)$ & $0.0433(17)$ & $0.0656(17)$ & $-0.0103(14)$ & $0.0226(14)$ & $-0.0036(13)$ \\
\hline$C(1)$ & $0.0451(17)$ & $0.054(2)$ & $0.0565(19)$ & $-0.0007(17)$ & $0.0186(15)$ & $0.0079(19)$ \\
\hline$c(2)$ & $0.058(2)$ & $0.063(3)$ & $0.074(2)$ & $-0.0089(19)$ & $0.0239(19)$ & $0.010(2)$ \\
\hline$C(3)$ & $0.060(2)$ & $0.096(3)$ & $0.072(2)$ & $-0.004(2)$ & $0.0304(19)$ & $0.022(3)$ \\
\hline$C(4)$ & $0.062(2)$ & $0.095(3)$ & $0.063(2)$ & $0.011(2)$ & $0.0296(19)$ & $0.004(2)$ \\
\hline$c(5)$ & $0.055(2)$ & $0.066(2)$ & $0.060(2)$ & $0.0055(18)$ & $0.0231(18)$ & $0.0003(19)$ \\
\hline$C(6)$ & $0.0418(17)$ & $0.046(2)$ & $0.0480(18)$ & $0.0028(15)$ & $0: 0123(15)$ & $0.0034(16)$ \\
\hline$C(7)$ & $0.0419(17)$ & $0.039(2)$ & $0.0452(18)$ & $0.0016(15)$ & $0.0084(14)$ & $-0.0012(15)$ \\
\hline$C(8)$ & $0.0544(19)$ & $0.0353(19)$ & $0.0550(19)$ & $0.0016(15)$ & $0.0165(16)$ & $-0.0005(15)$ \\
\hline$C(9)$ & $0.0518(18)$ & $0.0322(19)$ & $0.0502(18)$ & $0.0042(14)$ & $0.0185(15)$ & $0.0032(14)$ \\
\hline$C(10)$ & $0.058(2)$ & $0.059(2)$ & $0.056(2)$ & $-0.0042(17)$ & $0.0229(17)$ & $-0.0098(17)$ \\
\hline$C(11)$ & $0.062(2)$ & $0.067(3)$ & $0.072(2)$ & $-0.0044(18)$ & $0.0307(19)$ & $-0.007(2)$ \\
\hline$C(12)$ & $0.082(3)$ & $0.066(2)$ & $0.067\{2\}$ & $0.006(2)$ & $0.0435(19)$ & $0.001(2)$ \\
\hline$c(13)$ & $0.086(3)$ & $0.061(3)$ & $0.053\langle 2\rangle$ & $0.003(2)$ & $0.024(2)$ & $-0.0061(18)$ \\
\hline$C(14)$ & $0.063(2)$ & $0.050(2)$ & $0.055(2)$ & $-0.0040(18)$ & $0.0180(17)$ & $-0.0014(18)$ \\
\hline$C(15)$ & $0.072(2)$ & $0.061(3)$ & $0.060(2)$ & $-0.0110(19)$ & $0.0082(18)$ & $0.0030(19)$ \\
\hline$C(16)$ & $0.134(4)$ & $0.096(4)$ & $0.100(3)$ & $-0.029(3)$ & $-0.037(3)$ & $-0.001(3)$ \\
\hline$C(17)$ & $0.128(4)$ & $0.166(5)$ & $0.085(3)$ & $-0.015(4)$ & $0.025(3)$ & $0.056(3)$ \\
\hline$C(18)$ & $0.071(2)$ & $0.041(2)$ & $0.070(2)$ & $0.0026(17)$ & $0.0308(18)$ & $-0.0079(17)$ \\
\hline$C(19)$ & $0.104(3)$ & $0.047(2)$ & $0.096(3)$ & $0.004(2)$ & $0.043(2)$ & $-0.013(2)$ \\
\hline$C(20)$ & $0.122(3)$ & $0.082(3)$ & $0.142(4)$ & $-0.001(3)$ & $0.093(3)$ & $-0.016(3)$ \\
\hline$C(21)$ & $0.069(2)$ & $0.059(2)$ & $0.078(2)$ & $-0.0043(19)$ & $0.038(2)$ & $-0.003(2)$ \\
\hline$c(22)$ & $0.106(3)$ & $0.102(4)$ & $0.150(4)$ & $0.013(3)$ & $0.088(3)$ & $0.010(3)$ \\
\hline$C(23)$ & $0.120(3)$ & $0.105(4)$ & $0.085\{3\}$ & $0.004(3)$ & $0.060(3)$ & $0.025(3)$ \\
\hline
\end{tabular}


Calculated coordinates and thermal parameters $\left(\dot{\mathrm{A}}^{2}\right)$ for hydrogen atoms

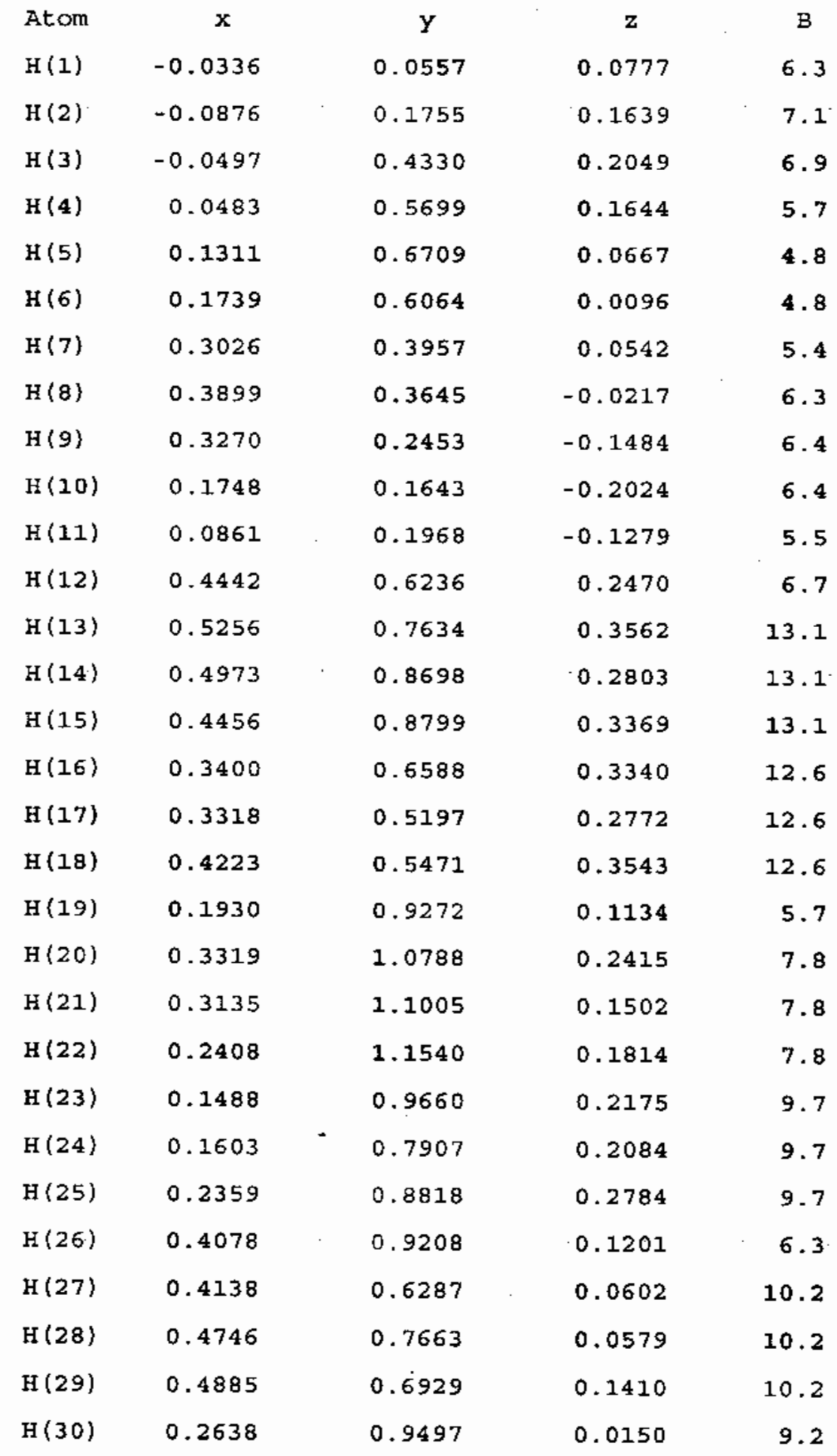


H (31)

H (32)

0.3384

0.9246

$-0.0183$

9.2

0. 7900

$-0.0189$

9.2 


\section{Supoorting data}

Intermolecular distances (A) for non-hydrogen atoms

\begin{tabular}{llllll}
$N(1)$ & $C\left(5^{i}\right)$ & $3.567(4)$ & $C(1)$ & $C\left(8^{i}\right)$ & $3.530(4)$ \\
$N(2)$ & $C\left(19^{i i}\right)$ & $3.524(5)$ & $C(2)$ & $C\left(8^{i}\right)$ & $3.572(4)$ \\
$N(2)$ & $C\left(2^{i i i}\right)$ & $3.590(5)$ & $C(6)$ & $C\left(7^{i}\right)$ & $3.531(4)$ \\
& \multicolumn{5}{c}{ Symunetry code }
\end{tabular}

(i) $-x, 1-y,-z$; (ii) $x,-1+y, z$; (iii) $-x,-y,-z$ 


\section{Torsion angles $\left({ }^{\circ}\right)$}

\begin{tabular}{|c|c|c|c|c|c|c|c|c|c|}
\hline (1) & (2) & (3) & (4) & angle & (1) & (2) & (3) & (4) & angle \\
\hline$S i$ & 0 & $C(8)$ & $C(7)$ & $-163.5(2)$ & $C(3)$ & $C(2)$ & $C(1)$ & $C(6)$ & $-0.1(5)$ \\
\hline 0 & Si & $C(15)$ & $\mathrm{C}(16)$ & $-179.3(3)$ & $C(3)$ & $C(4)$ & $C(5)$ & $C(6)$ & $0.2(5)$ \\
\hline 0 & $S i$ & $c(15)$ & $\mathrm{C}(17)$ & $-45.9(3)$ & $C(4)$ & $C(5)$ & $C(6)$ & $c(7)$ & $179.5(3)$ \\
\hline 0 & si & $C(18)$ & $C(19)$ & $-170.3(2)$ & $c(5)$ & $C(6)$ & $C(7)$ & $C(8)$ & $-4.3(5)$ \\
\hline 0 & Si & $C(18)$ & $c(20)$ & $61.9(3)$ & $C(6)$ & $C(7)$ & $\mathbf{N}(1)$ & $C\langle 9\}$ & $-178.0(3)$ \\
\hline 0 & $\mathrm{Si}$ & $C(21)$ & $c(22)$ & $-62.7(3)$ & $C(7)$ & $N(1)$ & $c(9)$ & $c(10)$ & $44.5(4)$ \\
\hline 0 . & Si & $C(21)$ & $\mathrm{C}(23)$ & $64.1(3)$ & $C(7)$ & $N(2)$ & $C(9)$ & $C(14)$ & $-137.8(3)$ \\
\hline 0 & $C(8)$ & $C(7)$ & $N(1)$ & $-93.2(3)$ & $C(8)$ & 0 & $\mathrm{Si}$ & $c(15)$ & $163.8(2)$ \\
\hline 0 & $C(8)$ & $C(7)$ & $C(6)$ & $93.7(3)$ & $C(8)$ & 0 & Si & $C(18)$ & $39.7(2)$ \\
\hline $\mathbf{N}(1)$ & $N(2)$ & $c(1)$ & $C(2)$ & $-180.0(3)$ & $C(8)$ & 0 & Si & $c(21)$ & $-79.3(2)$ \\
\hline $\mathbf{N}(1)$ & $\mathbf{N}(2)$ & $C(1)$ & $c(6)$ & $0.5(3)$ & $C(8)$ & $C(7)$ & $N(1)$ & $C(9)$ & $7.6(4)$ \\
\hline $\mathbf{N}(1)$ & $C(7)$ & $C(6)$ & $C(1)$ & $0.2(3)$ & $C(9)$ & $C(10)$ & $\mathrm{C}(11)$ & $C(12)$ & $-1.0(5)$ \\
\hline$N(1)$ & $C(7)$ & $C(6)$ & $C(5)$ & $-178.3(3)$ & $\mathrm{C}(9)$ & $C(14)$ & $\mathrm{C}(13)$ & $C(12)$ & $0.4(5)$ \\
\hline$N(1)$ & $C(9)$ & $C(10)$ & $\mathrm{C}(11)$ & $178.4(3)$ & $c(10)$ & $C(9)$ & $C(14)$ & $C(13)$ & $-0.3(5)$ \\
\hline$N(1)$ & $C(9)$ & $C(14)$ & $c(13)$ & $-178.1(3)$ & $c(10)$ & ) $C(11)$ & $C(12)$ & $C(13)$ & $1.1(6)$ \\
\hline$N(2)$ & $N(1)$ & $C(7)$ & $C(6)$ & $0.0(3)$ & $C(11)$ & ) $C(10)$ & $C(9)$ & $C(14)$ & $0.7(5)$ \\
\hline$N\langle 2\rangle$ & $N(1)$ & $C(7)$ & $C(8)$ & $-174.4(2)$ & $C(11)$ & )$C(12)$ & $C(13)$ & $C(14)$ & $-0.7(5)$ \\
\hline$N\langle 2\rangle$ & $N(1)$ & $c(9)$ & $C(10)$ & $-133.5(3)$ & $c(15)$ & Si & $C(18)$ & $C(19)$ & $74.9(3)$ \\
\hline$N(2)$ & $N(1)$ & $C(9)$ & $C(14)$ & $44.3(4)$ & $C(15)$ & Si & $C(18)$ & $C(20)$ & $-52.9(3)$ \\
\hline$N(2)$ & $C(1)$ & $c(2)$ & $C(3)$ & $-179.6(3)$ & $C(15)$ & Si & $C(21)$ & $C(22)$ & $48.9(3)$ \\
\hline $\mathbf{N}(2)$ & $c(1)$ & $C(6)$ & $C(5)$ & $178.4(3)$ & $C(1.5)$ & $\mathrm{Si}$ & $C(21)$ & $C(23)$ & $175.7(3)$ \\
\hline$N(2)$ & $C(1)$ & $c(6)$ & $C(7)$ & $-0.5(3)$ & $c(16)$ & $\mathrm{C}(15)$ & $\mathrm{Si}$ & $C(18)$ & $-60.0(4)$ \\
\hline$c(1)$ & $N(2)$ & $N(1)$ & $C(7)$ & $-0.3(3)$ & $C(16)$ & ) C(15) & $\mathrm{Si}$ & $C(21)$ & $63.1(4)$ \\
\hline
\end{tabular}




\begin{tabular}{|c|c|c|c|c|c|c|c|c|}
\hline$C(1)$ & $N(2)$ & $N(1)$ & $c(9)$ & $177.9(2)$ & \multicolumn{2}{|c|}{$\mathrm{C}(17) \mathrm{C}(15) \mathrm{si}$} & $C(18)$ & $73.5(4)$ \\
\hline$c(1)$ & $C(2)$ & $C(3)$ & $C(4)$ & $1.4(5)$ & \multicolumn{2}{|c|}{$\mathrm{C}(17) \mathrm{C}(15) \mathrm{si}$} & $C(21)$ & $-163.5(3)$ \\
\hline$c(1)$ & $C(6)$ & $C(5)$ & $C(4)$ & $1.1(4)$ & $C(18) \mathrm{Si}$ & \multicolumn{2}{|c|}{$C(21) C(22)$} & $177.0(3)$ \\
\hline$C(1)$ & $C(6)$ & $C(7)$ & $C(8)$ & $174.3(3)$ & $\mathrm{C}(18) \mathrm{Si}$ & $\mathrm{C}(2$ & $\mathrm{C}(23)$ & $-56.1(3)$ \\
\hline$C(2)$ & $C(1)$ & $C(6)$ & $C(5)$ & $-1.2(4)$ & \multicolumn{2}{|c|}{$C(19) C(18) S i$} & $C(21)$ & $-49.4(3)$ \\
\hline$C(2)$ & $C(1)$ & $C(6)$ & $C(7)$ & $180.0(3)$ & \multicolumn{2}{|c|}{$\mathrm{C}(20) \mathrm{C}(18) \mathrm{Sj}$} & $C(21)$ & $-177.2(3)$ \\
\hline$C(2)$ & $C(3)$ & $C(4)$ & $C(5)$ & $-1.3(5)$ & & & & \\
\hline
\end{tabular}

The sign is positive if a clockwise motion of atom 1 would superimpose it on atom 4 when the direction of view is from atom 2 to atom 3 . 
Least-squares mean planes (deviations in $\dot{A}$ )

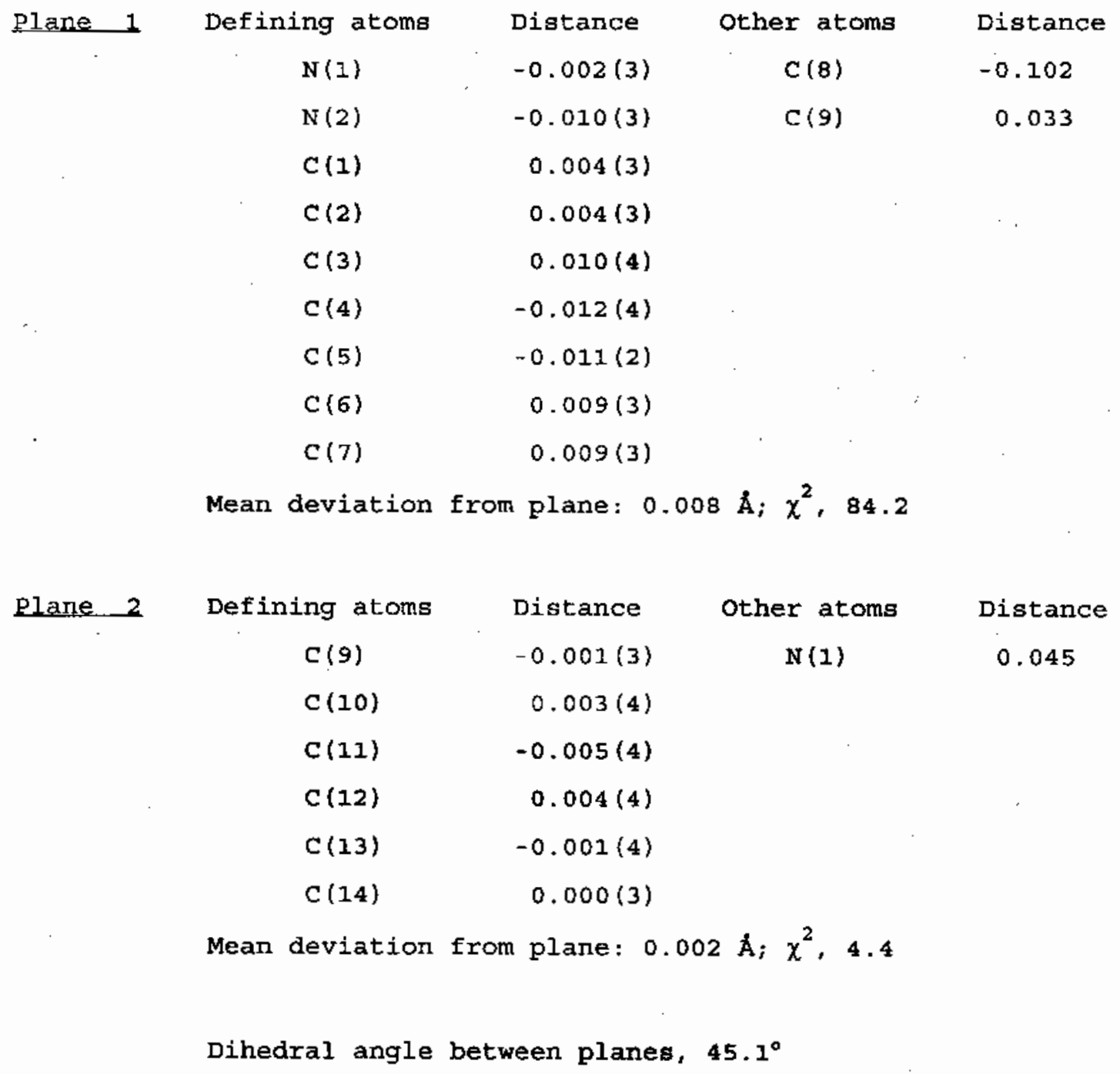




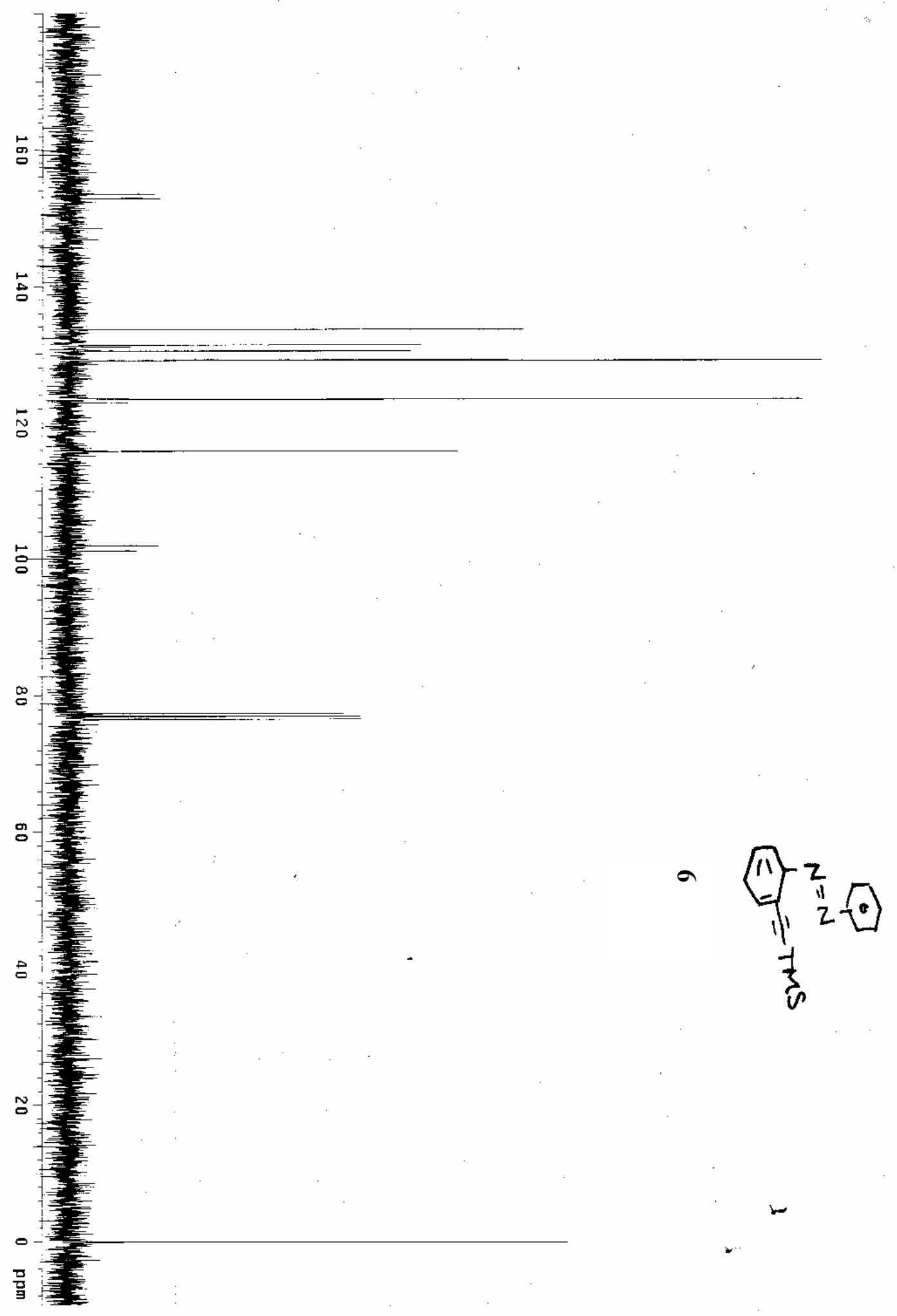




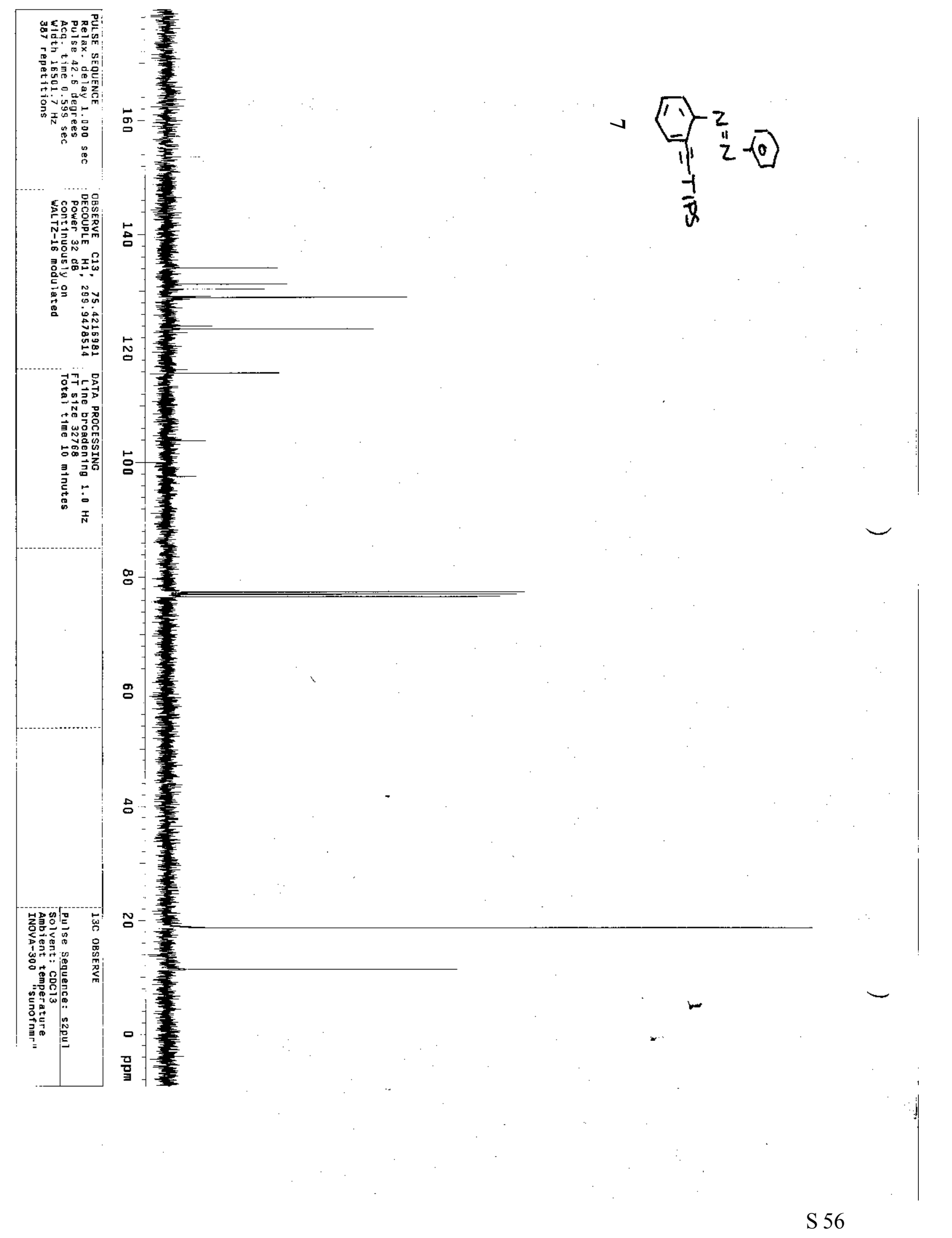




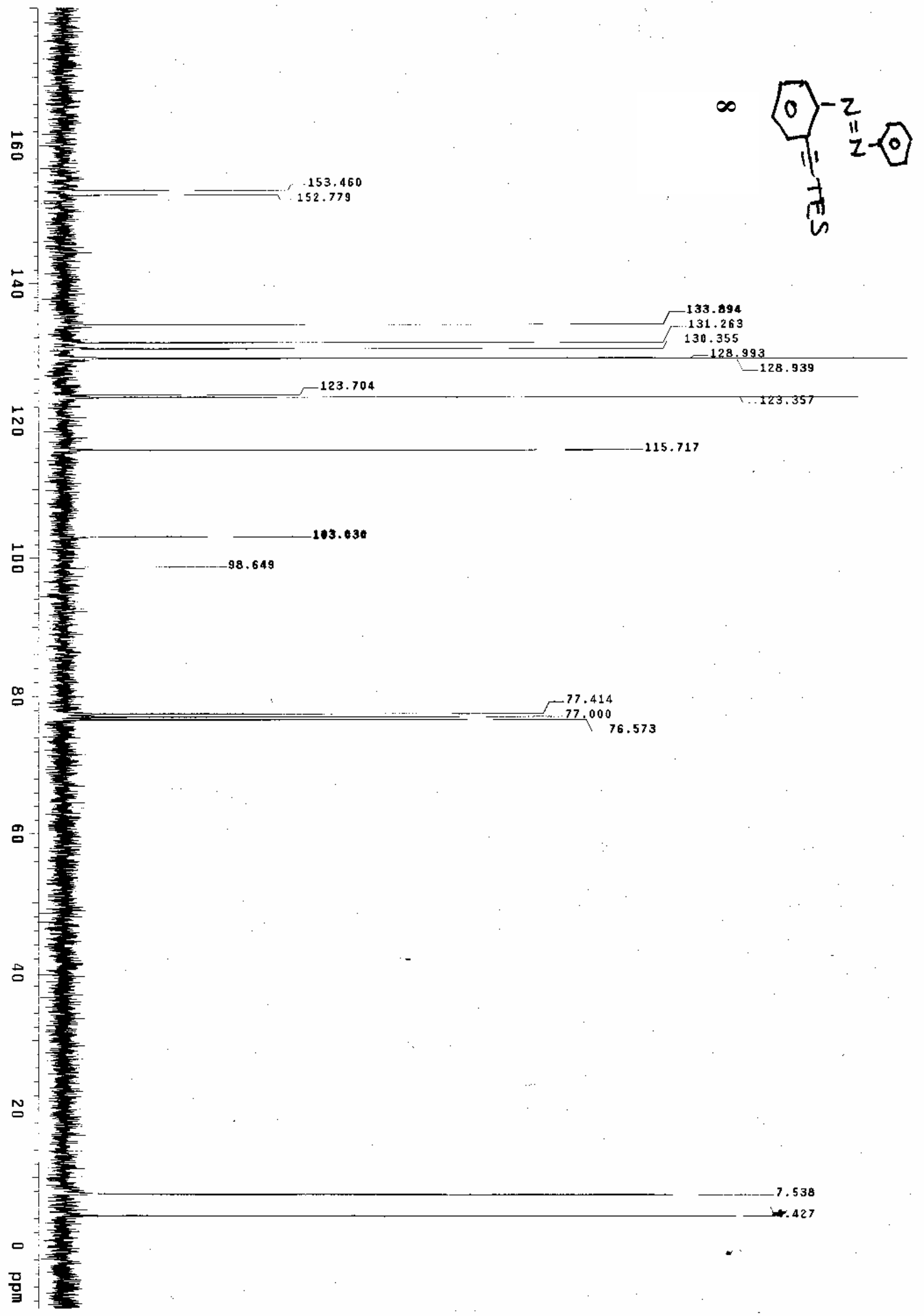




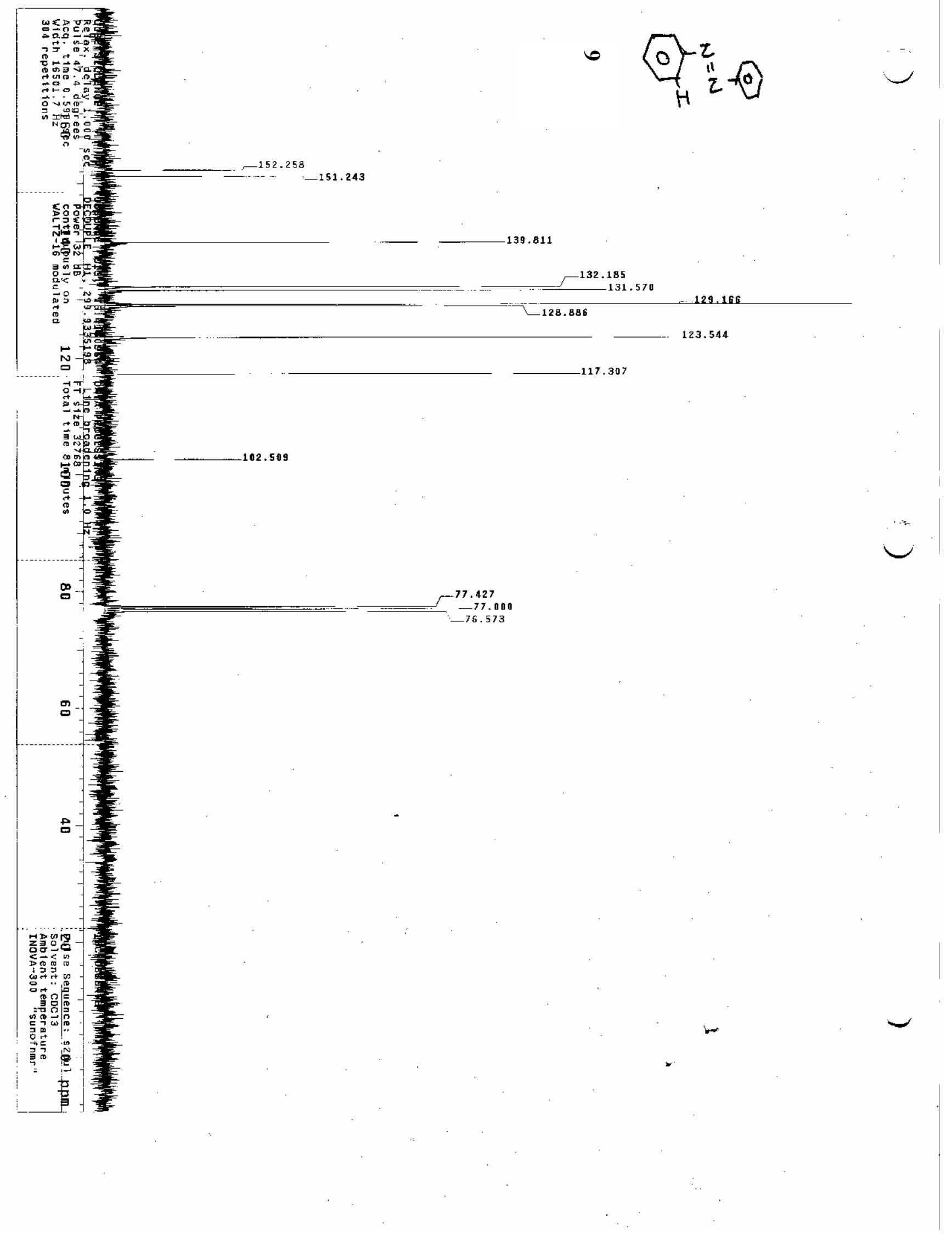




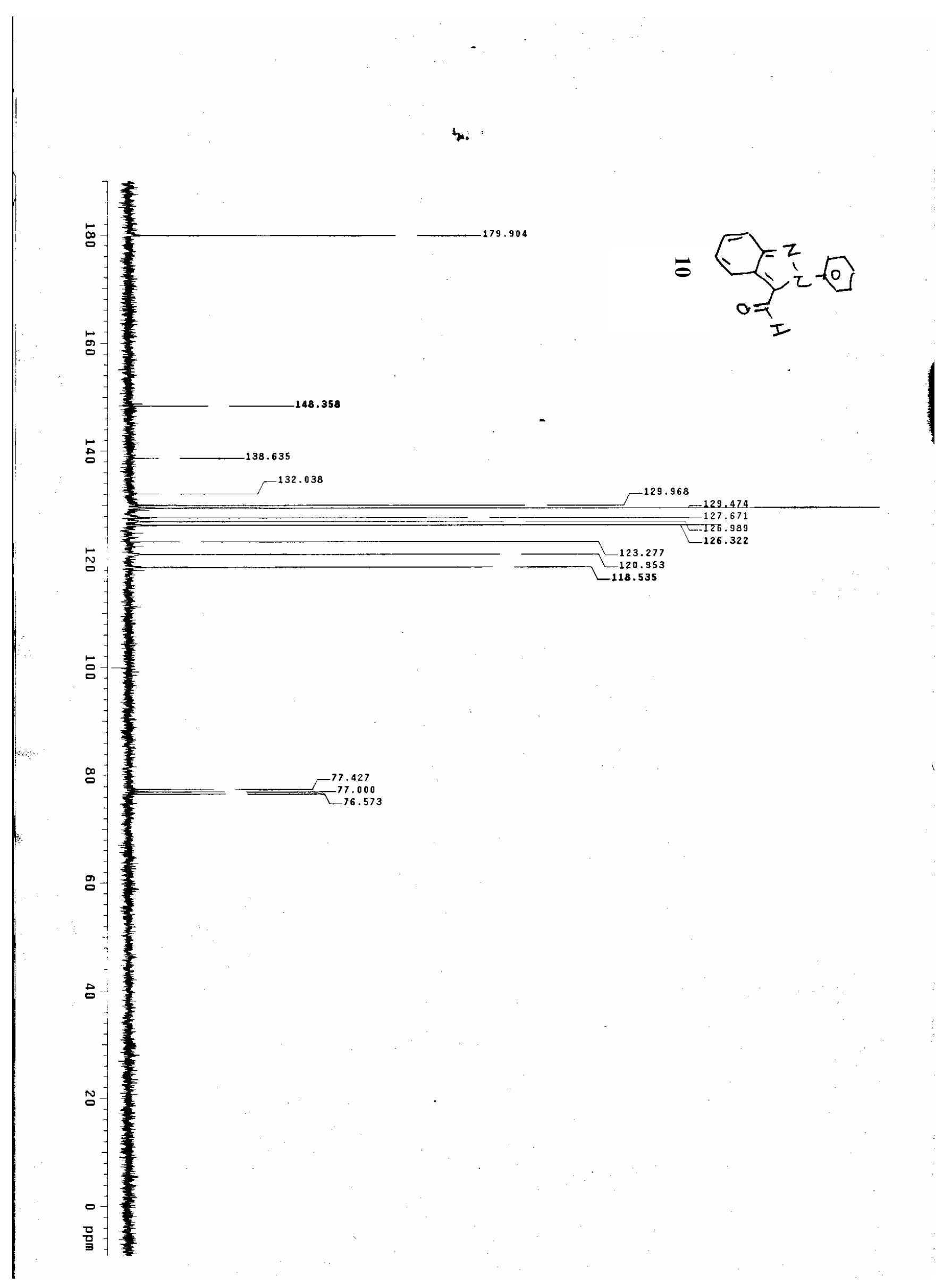




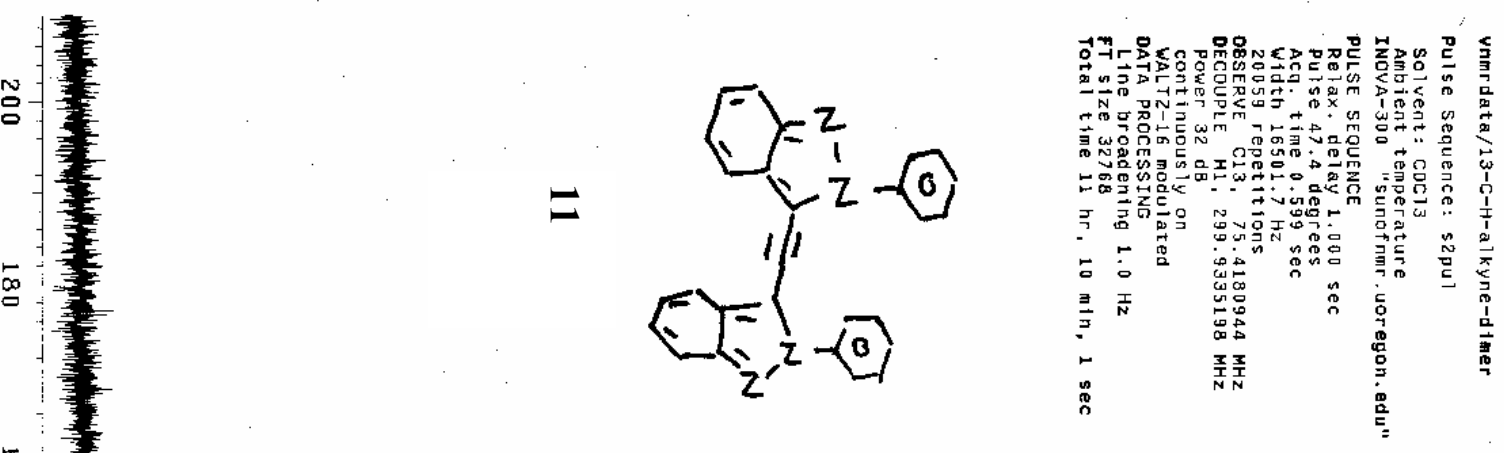

E.

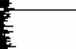

엄

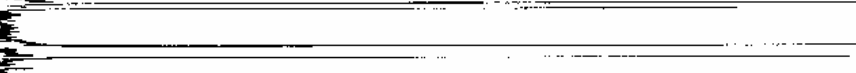

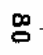

量

5

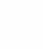

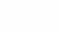

0

(

再 

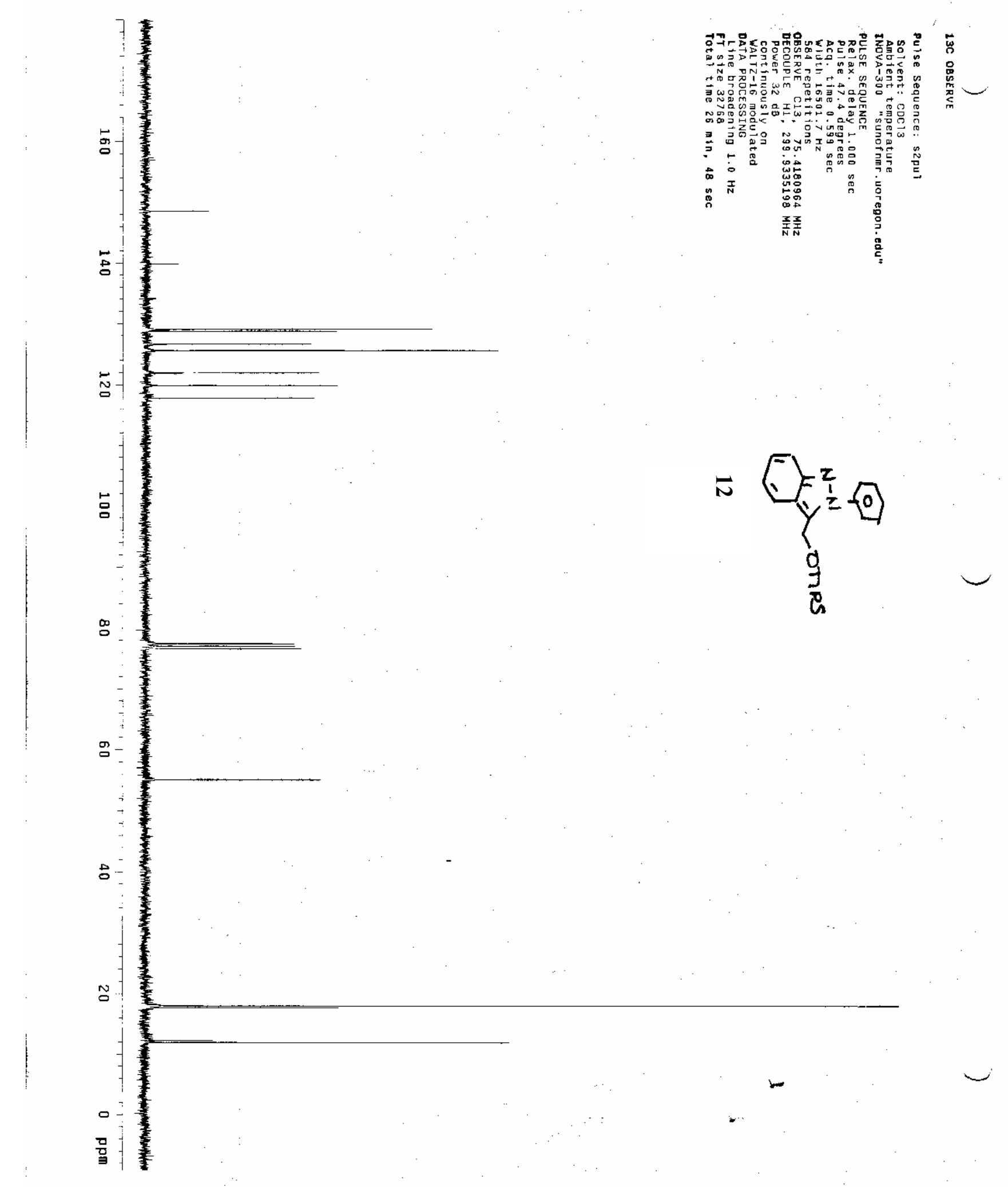


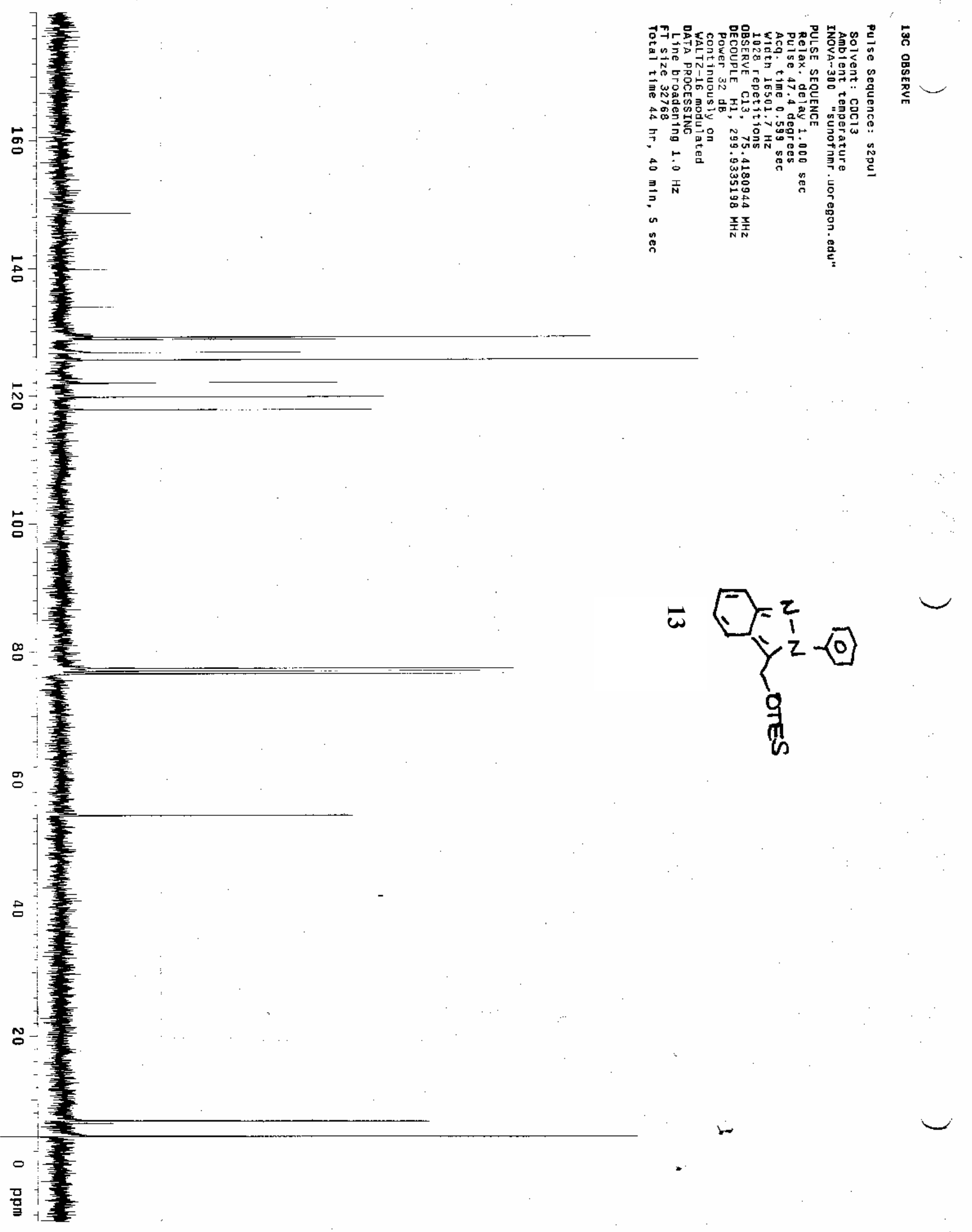




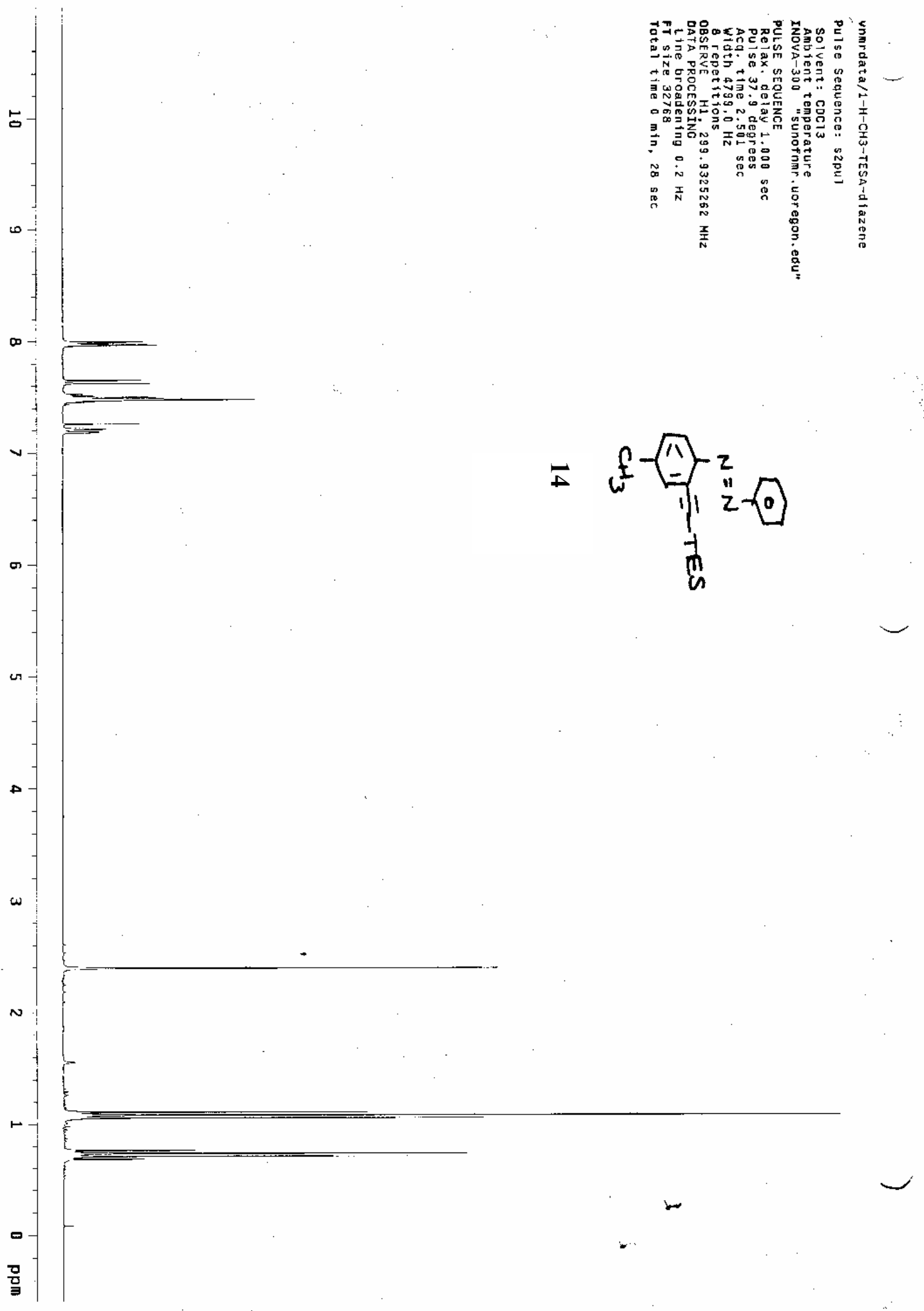




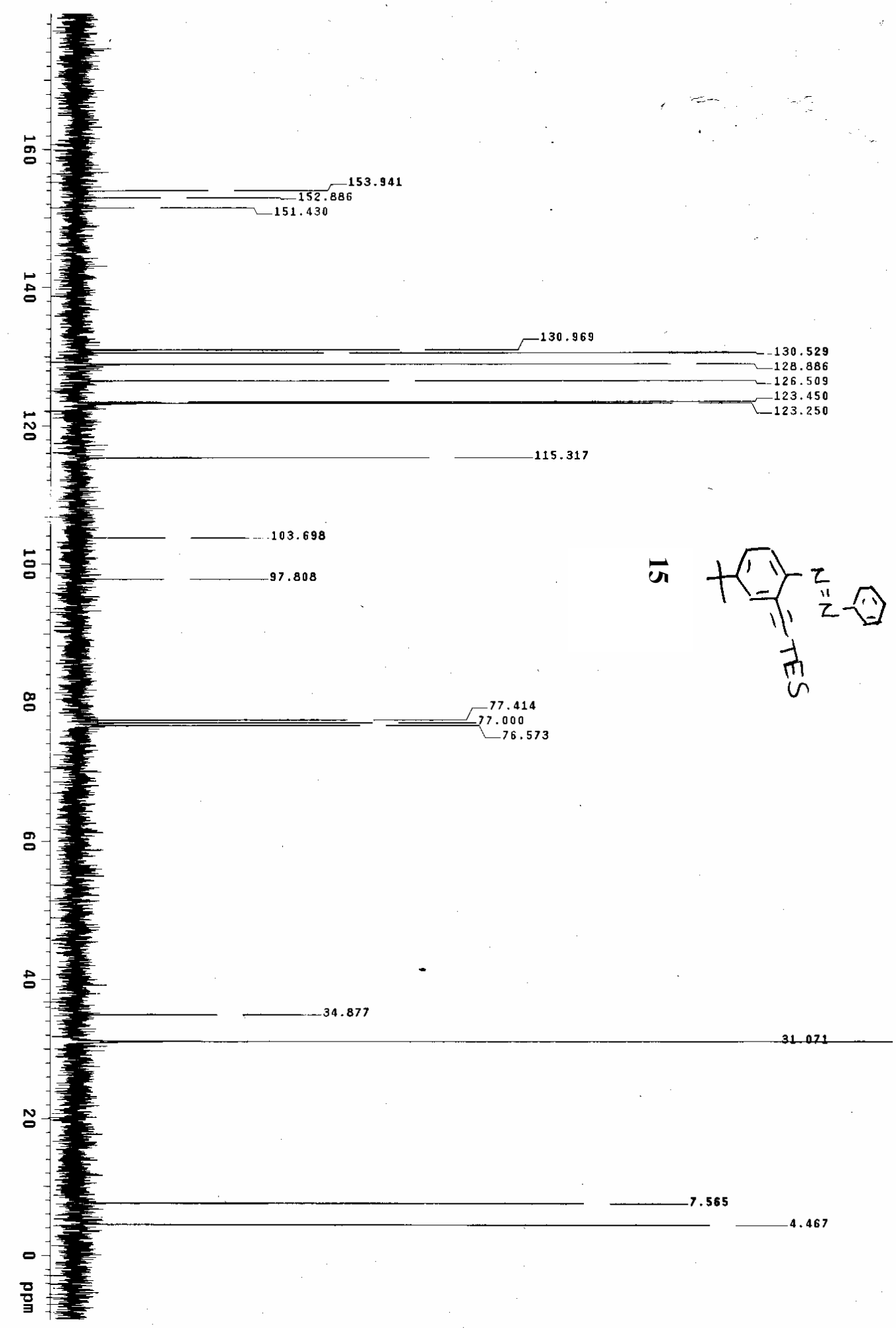




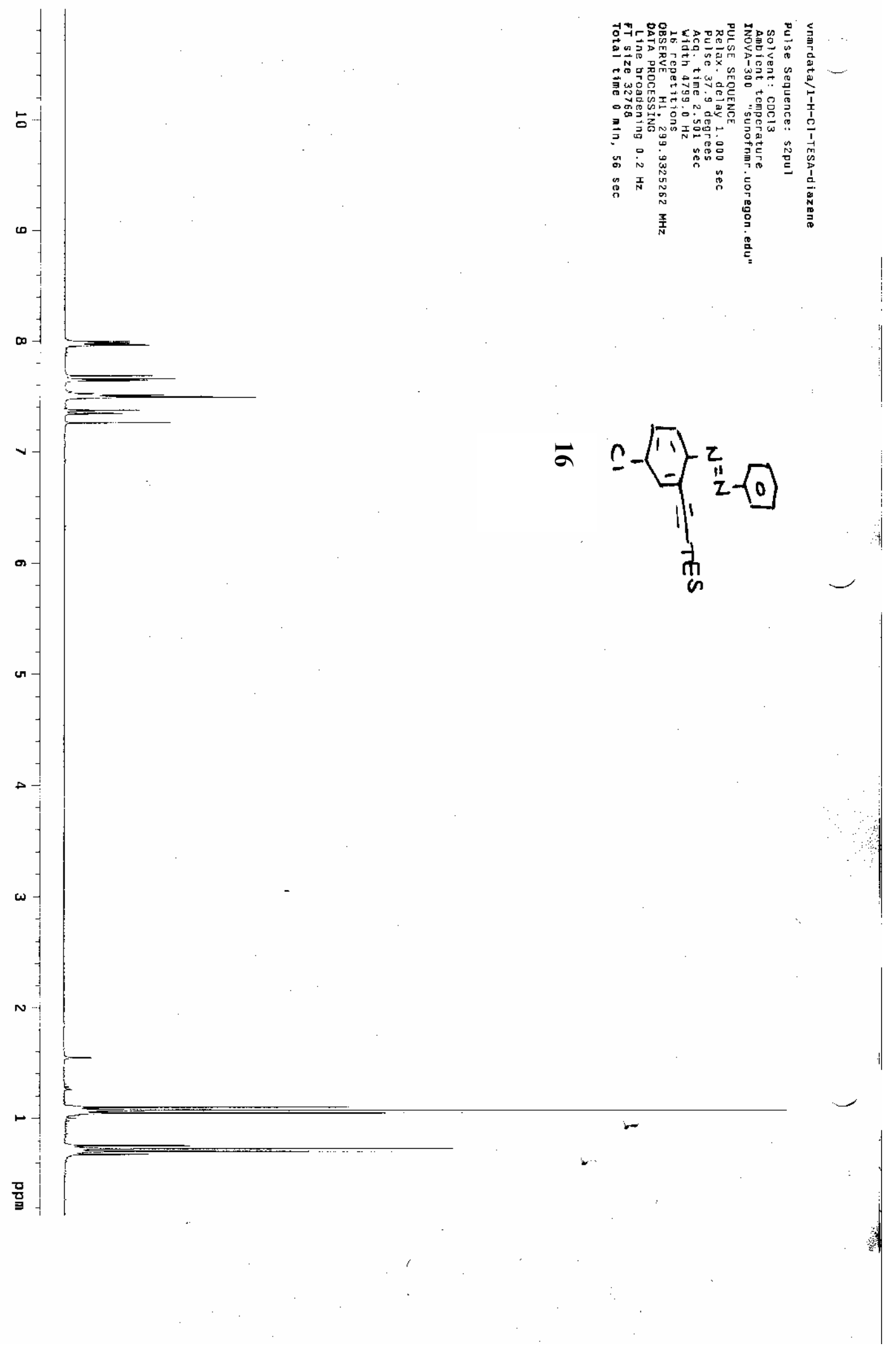




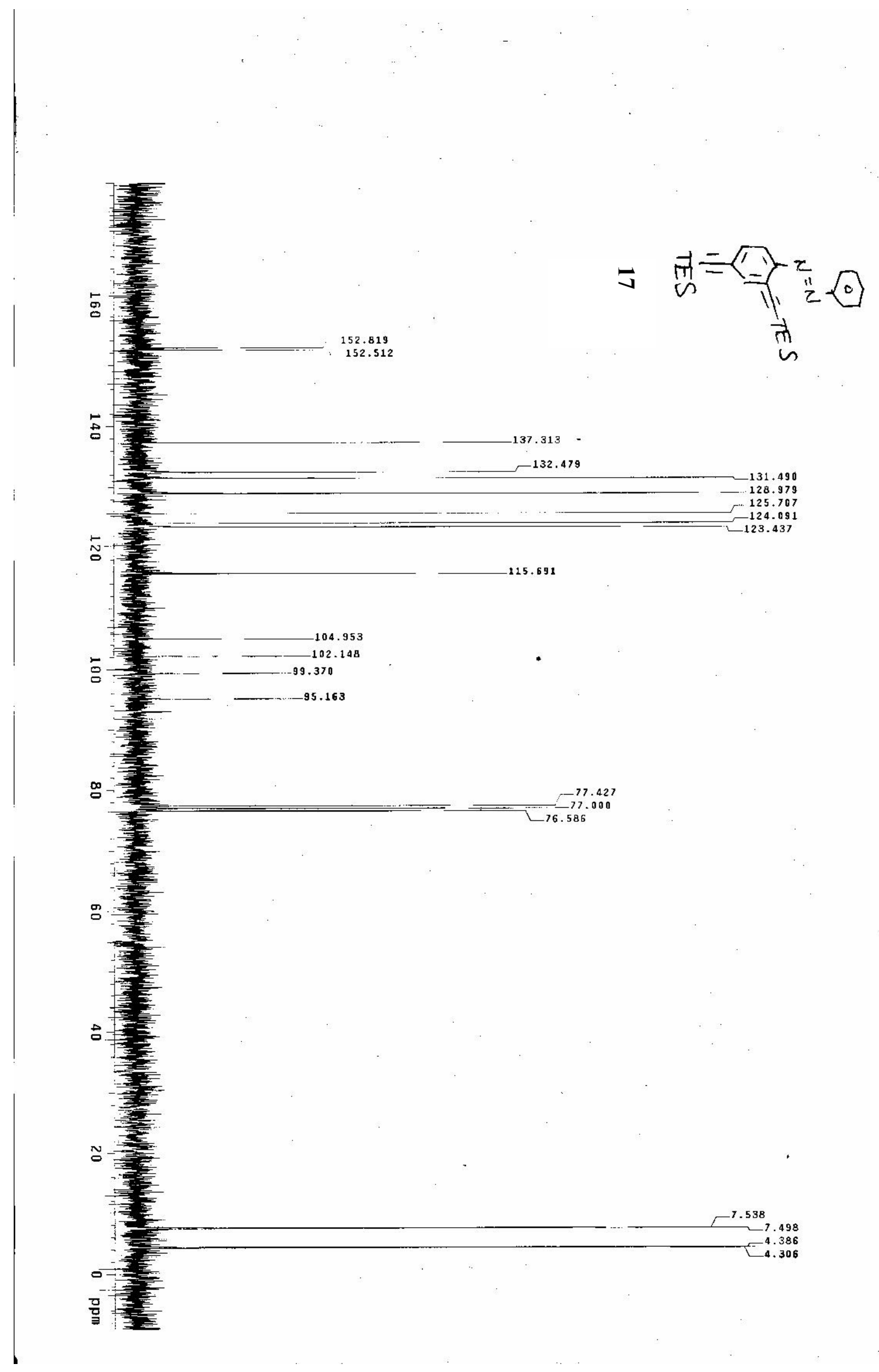



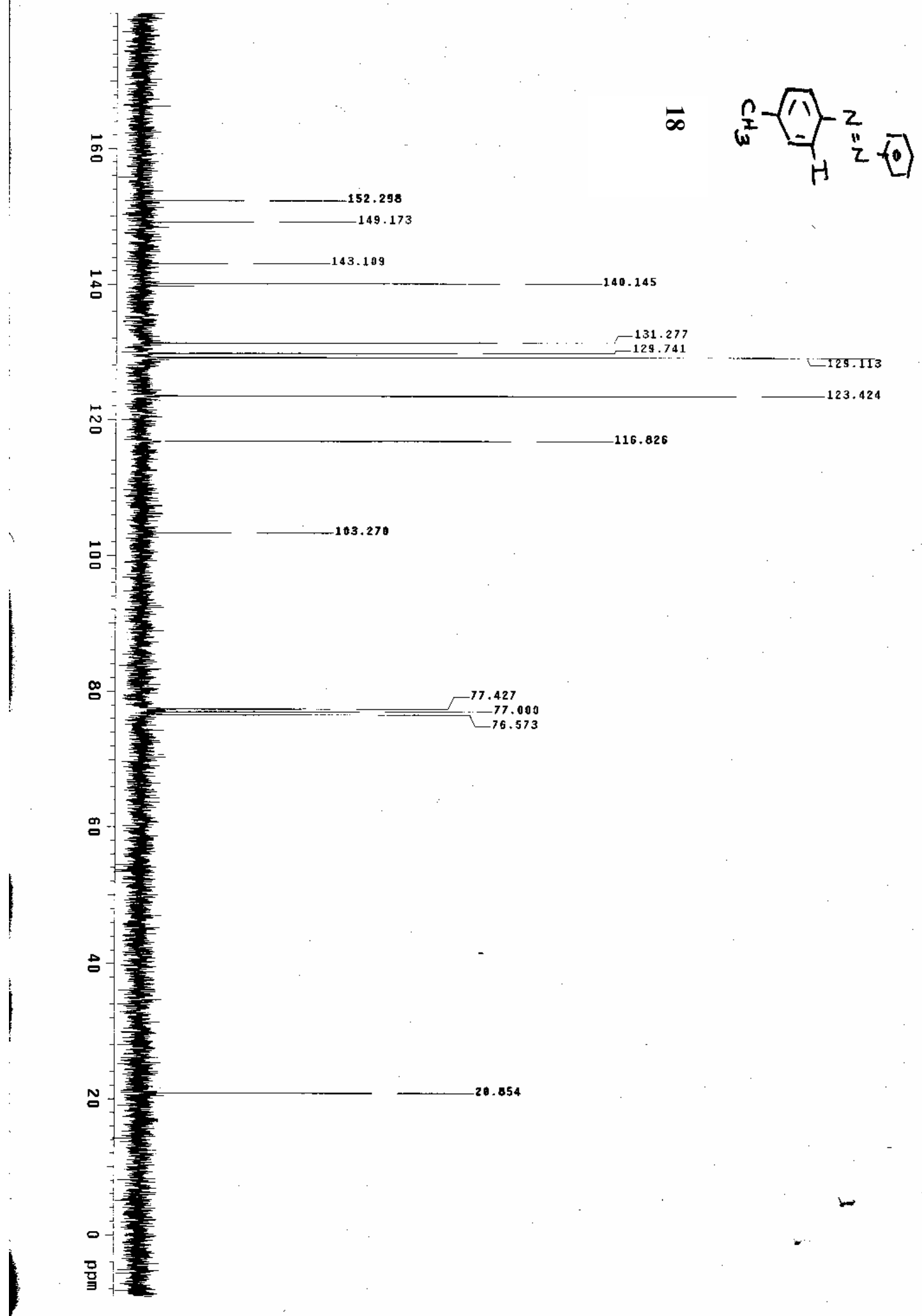


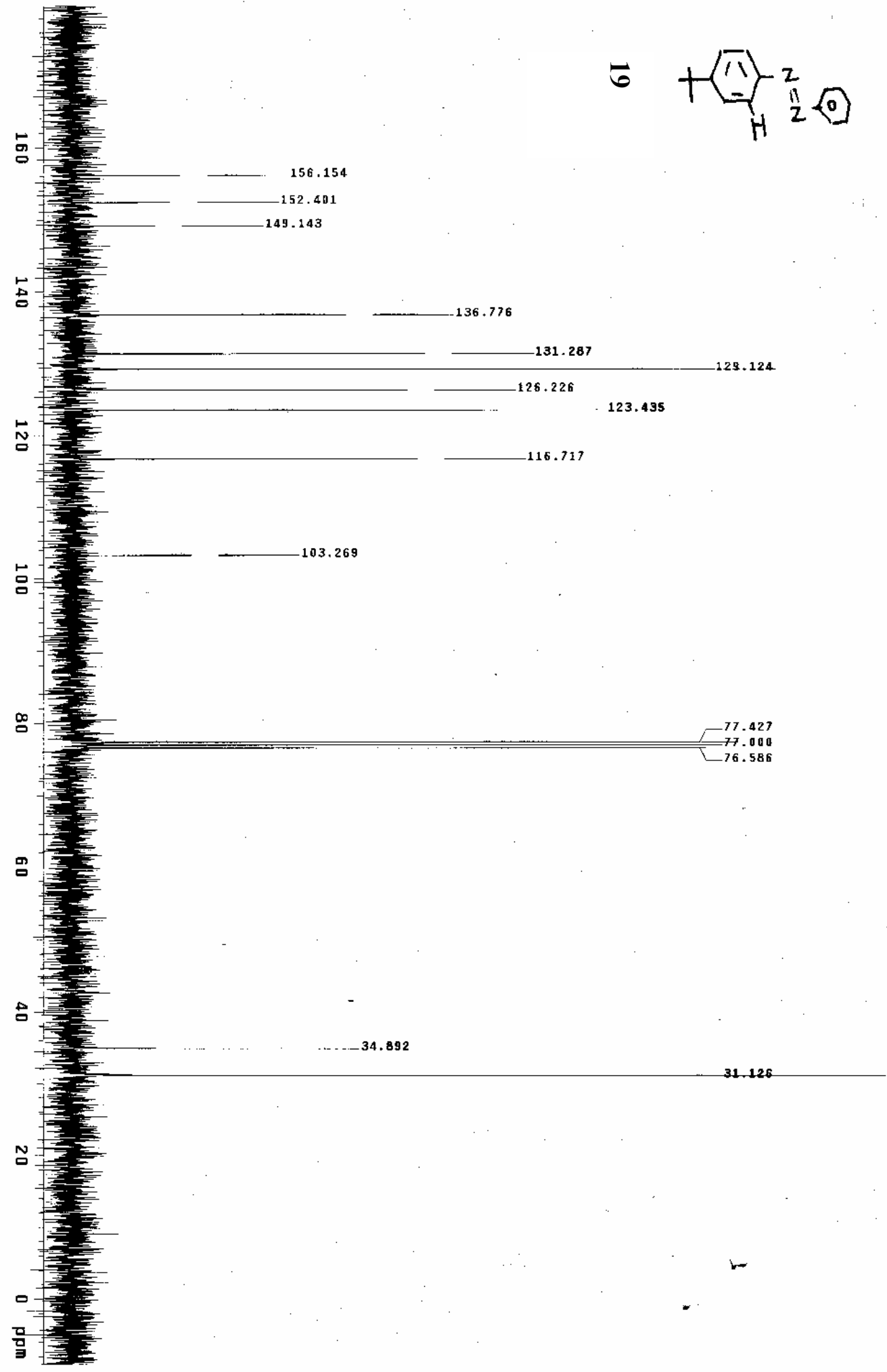




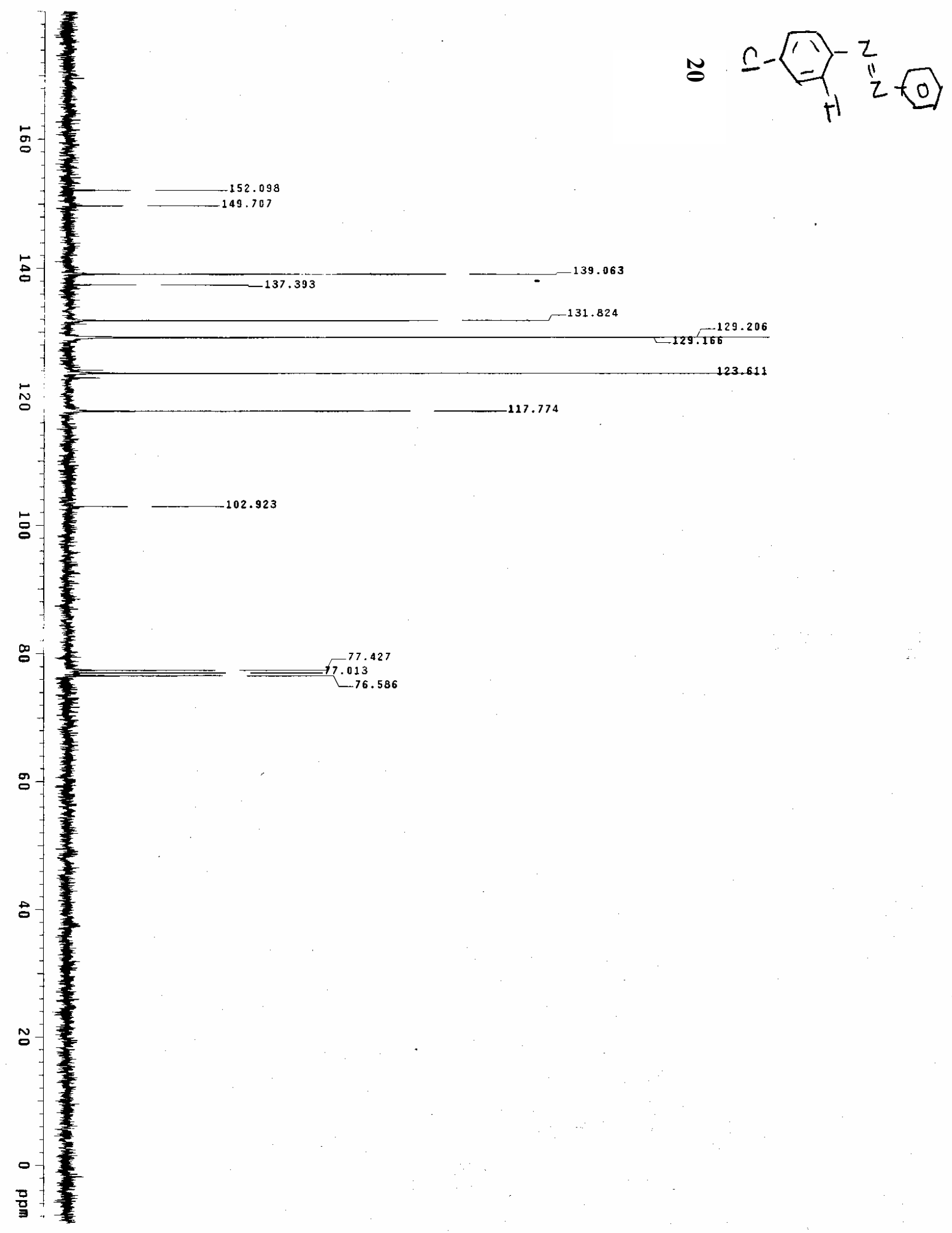




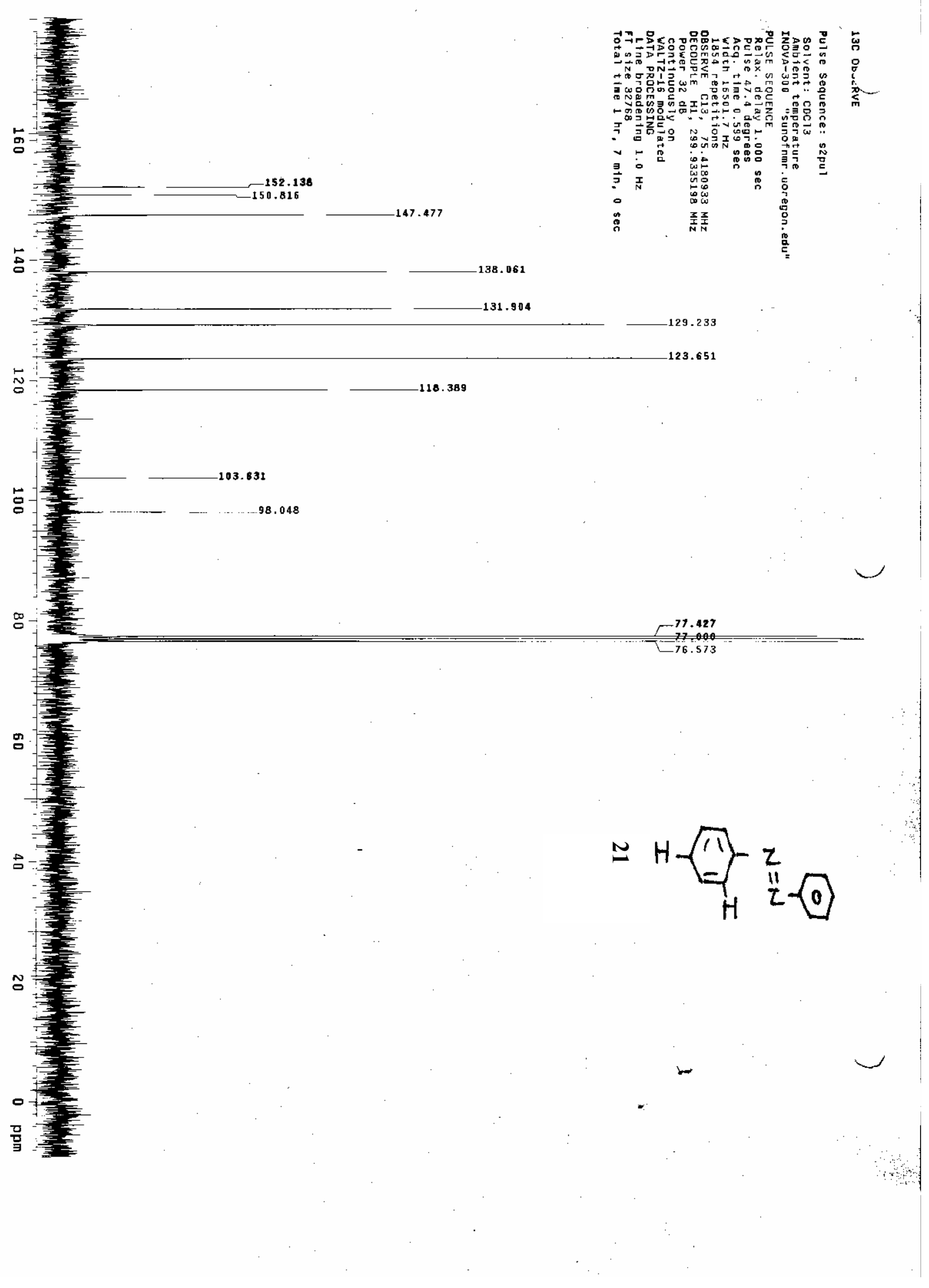




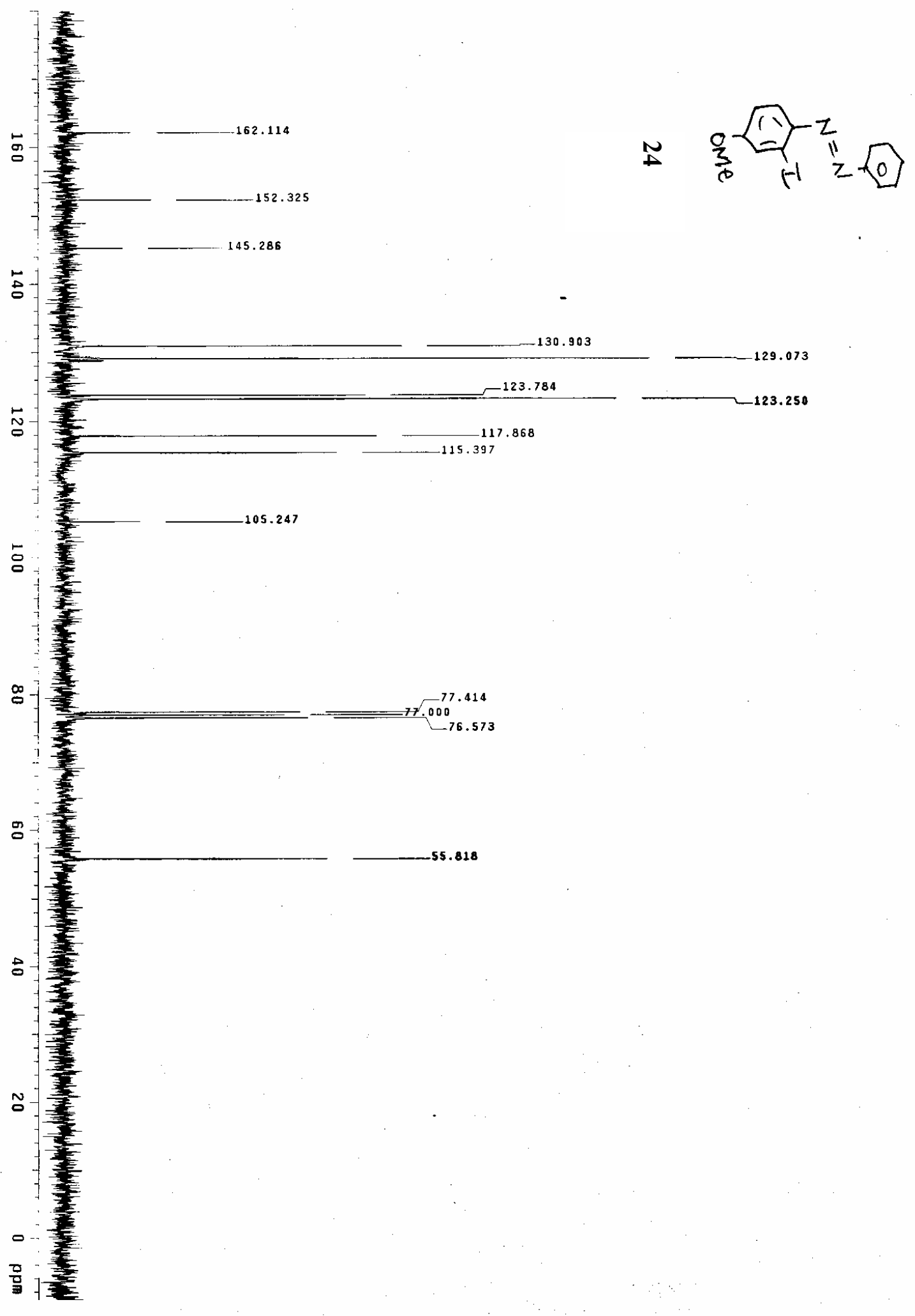



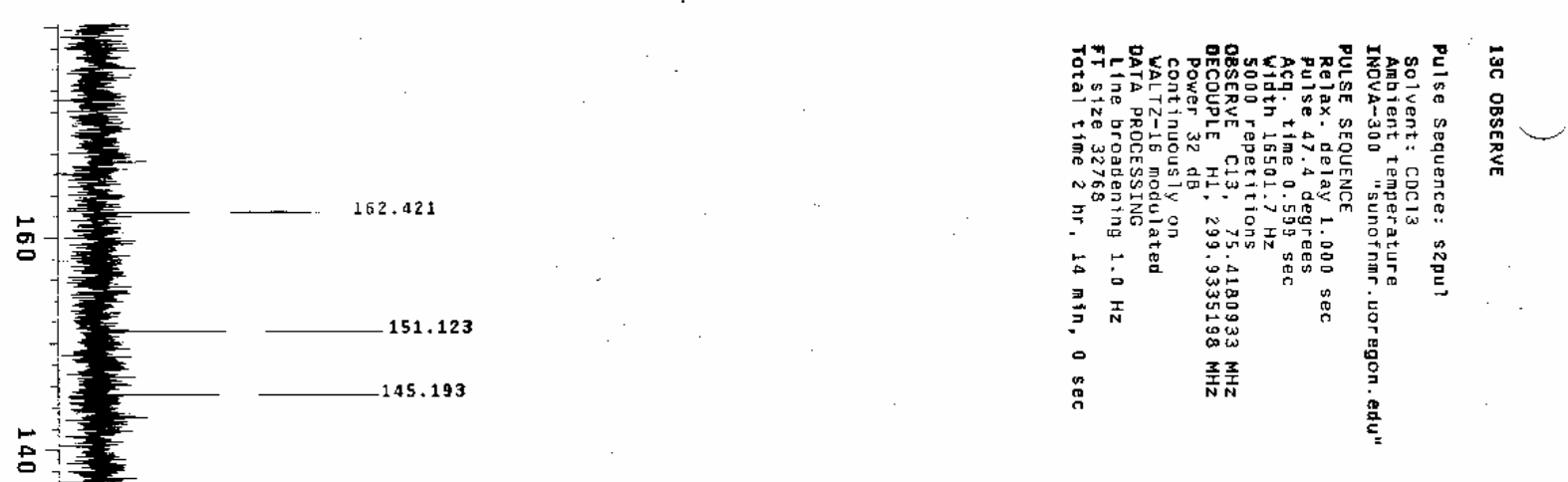

132.332

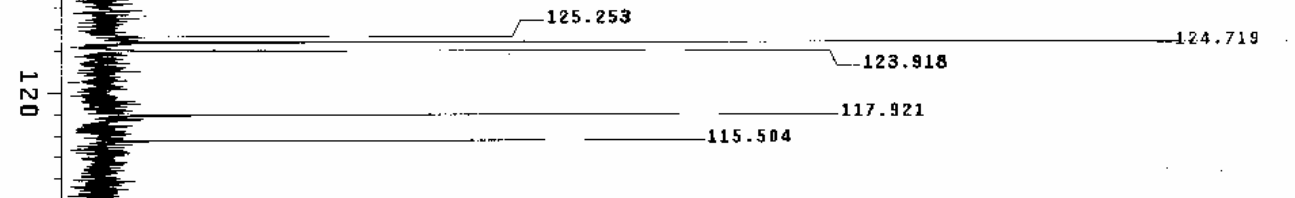

음

宫

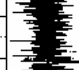

$-105.621$

몽

哭
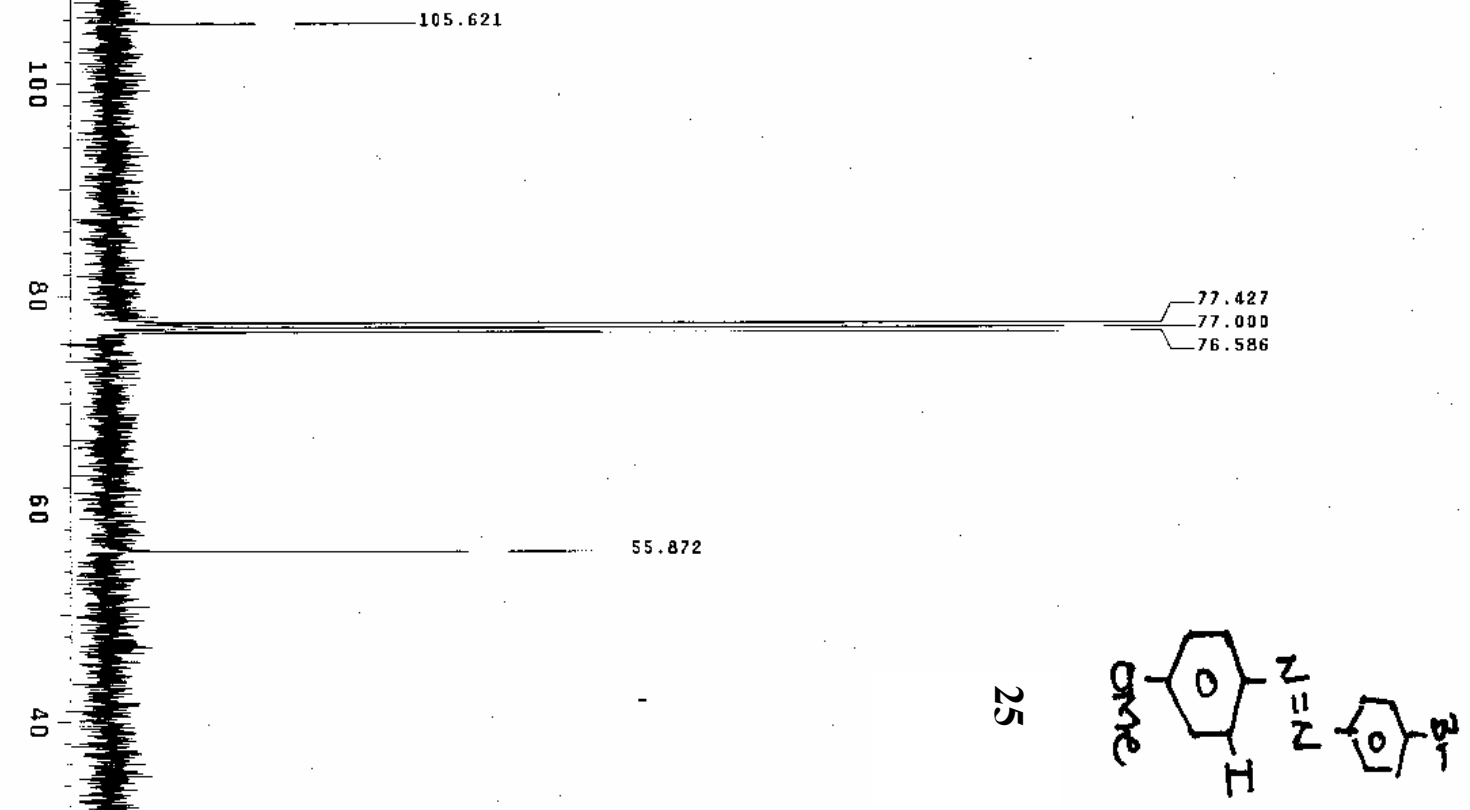

ㄱ-
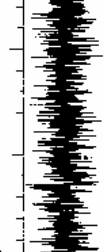


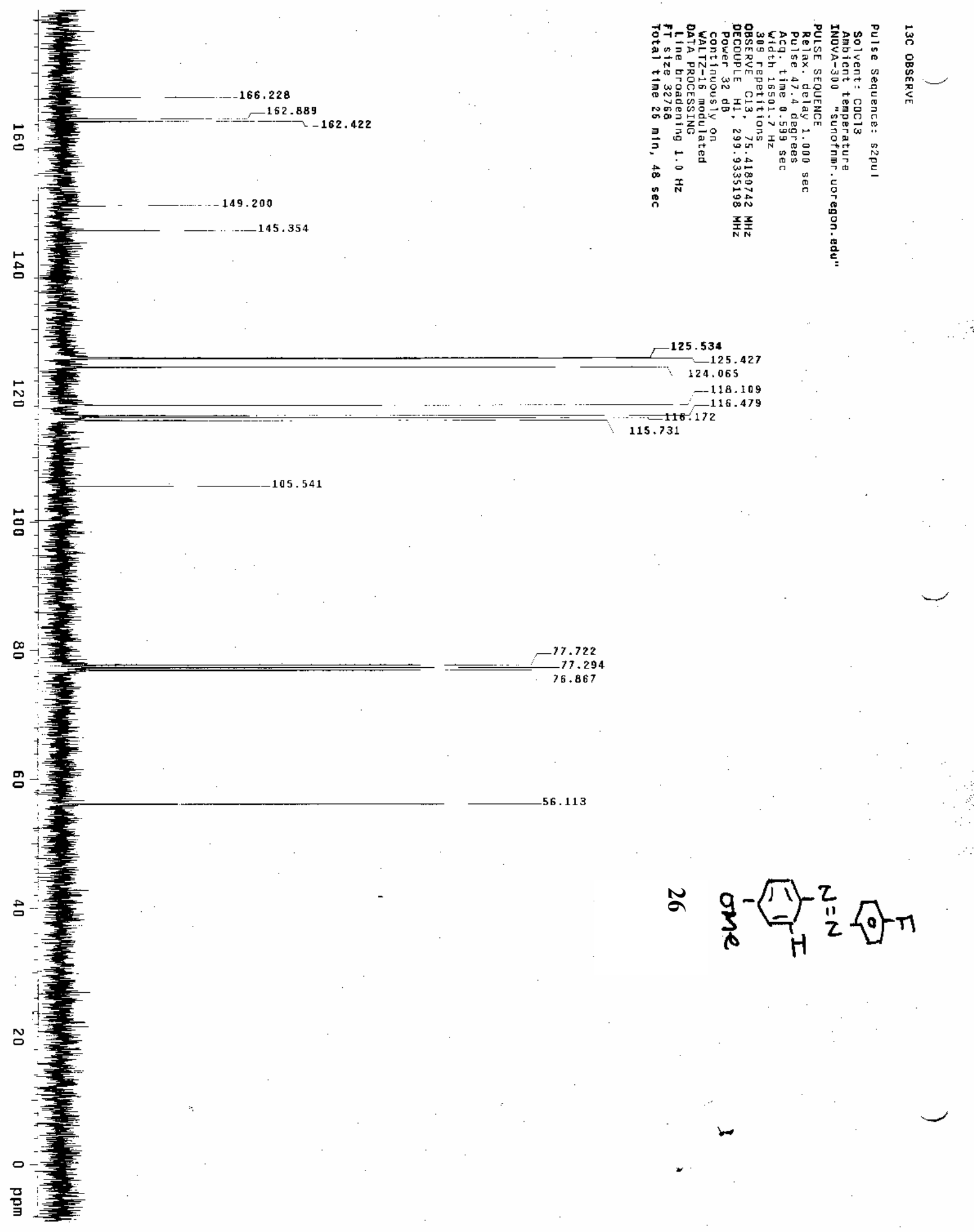




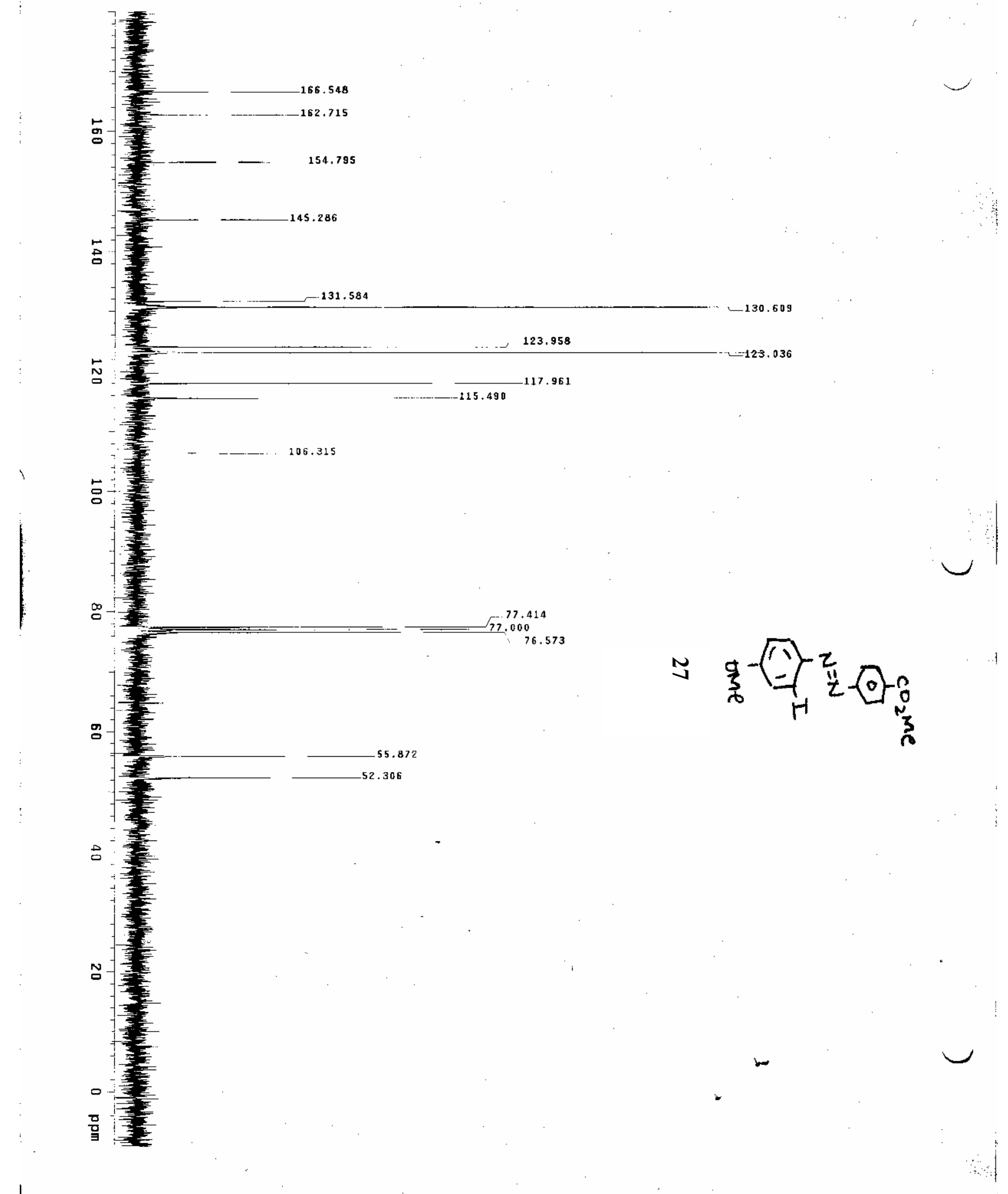




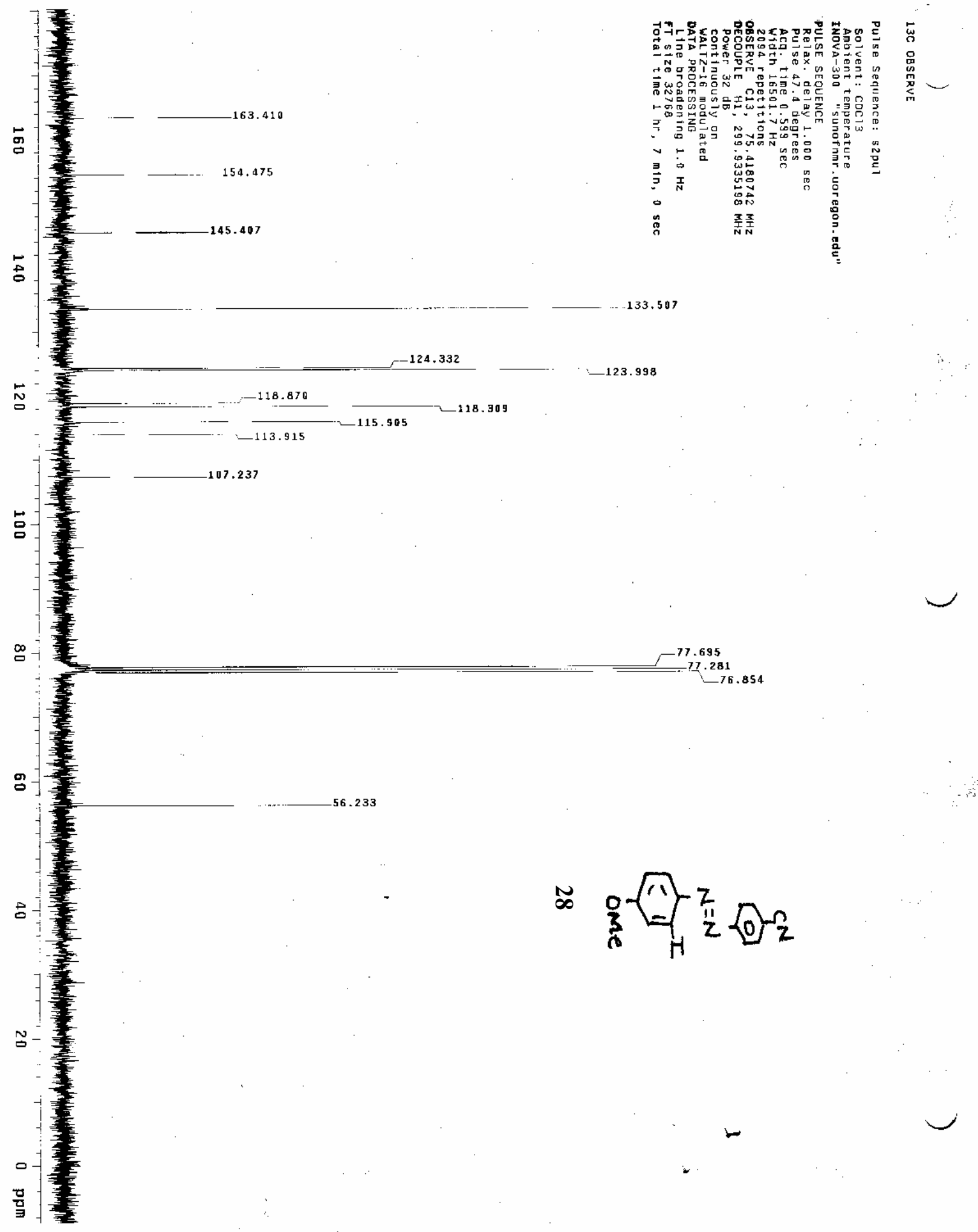



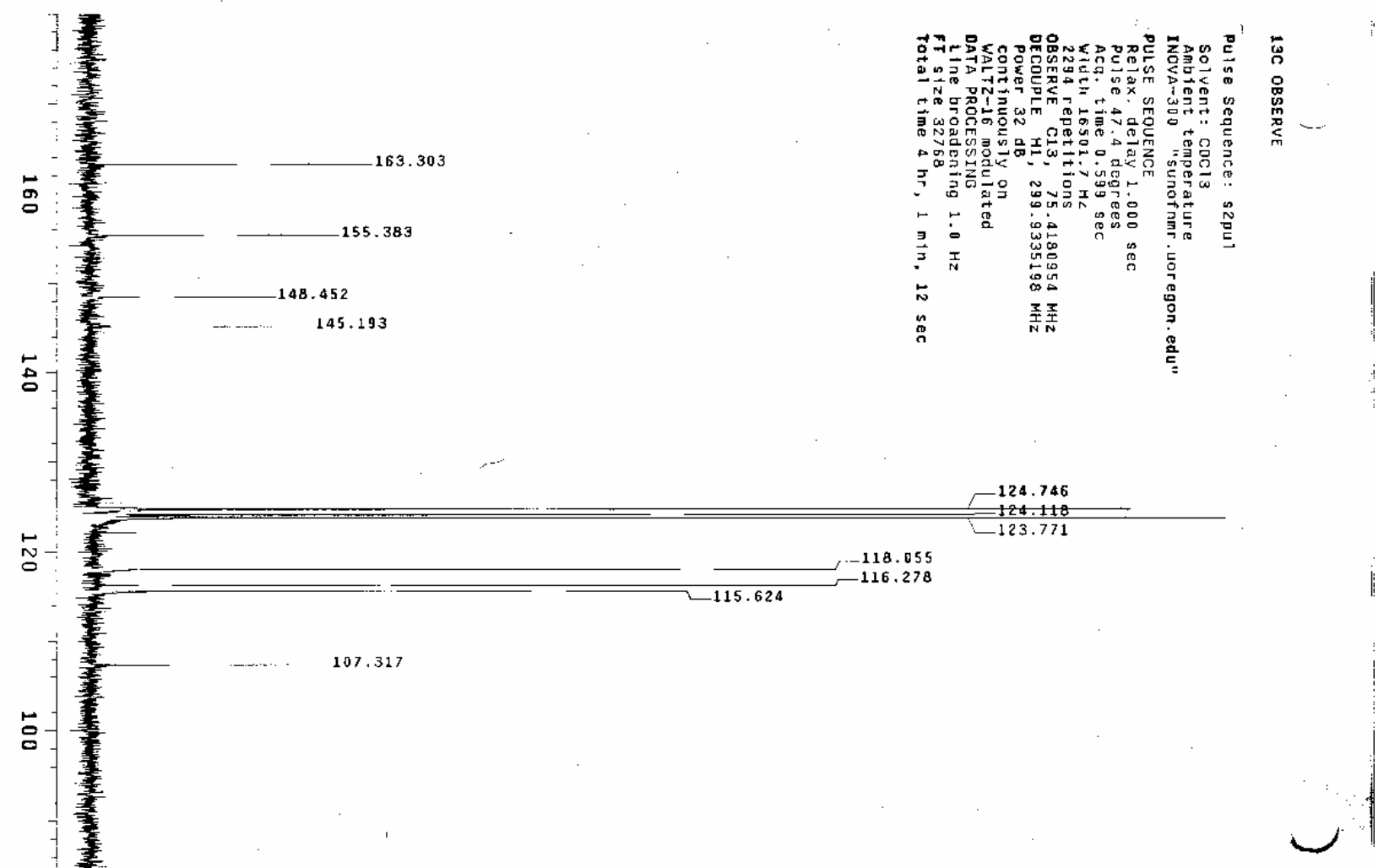

$\stackrel{\infty}{\square}$

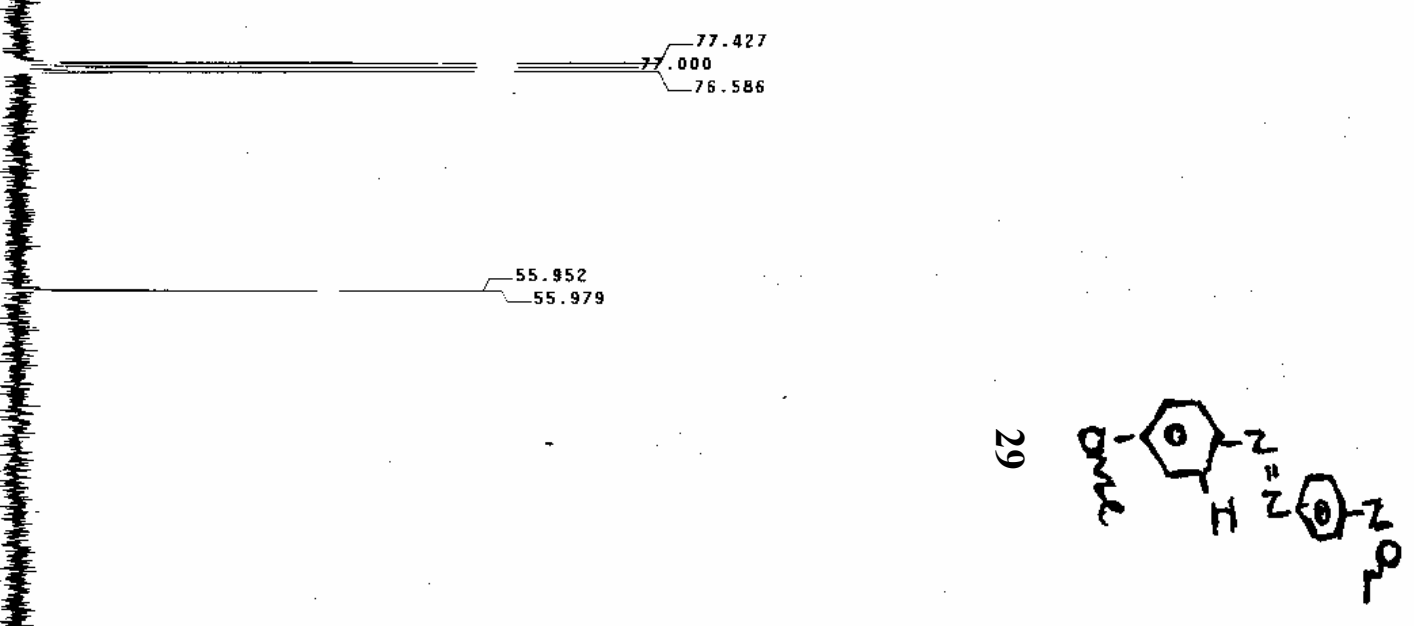

ㅇ.

.

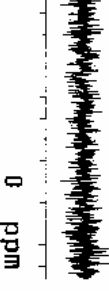




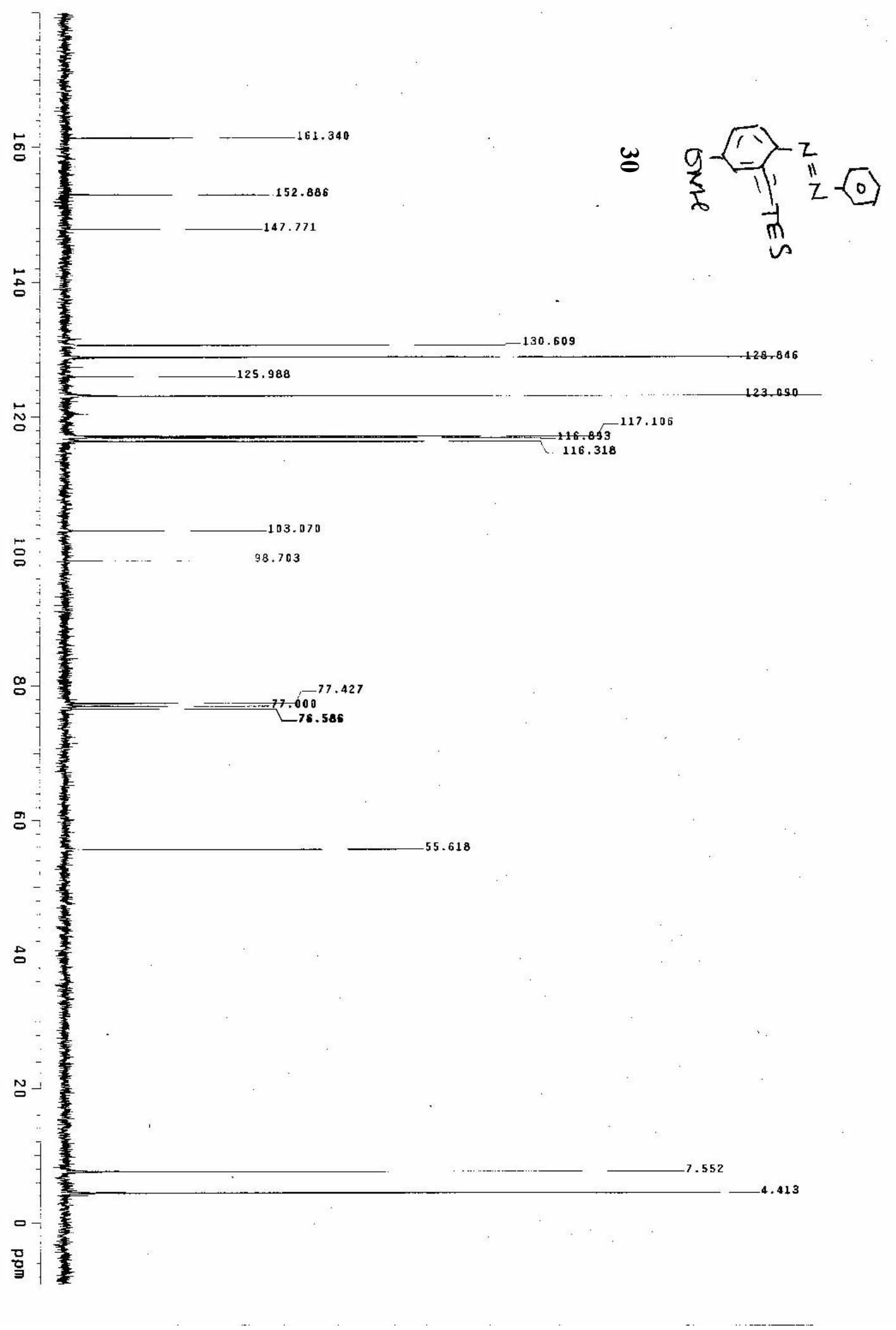




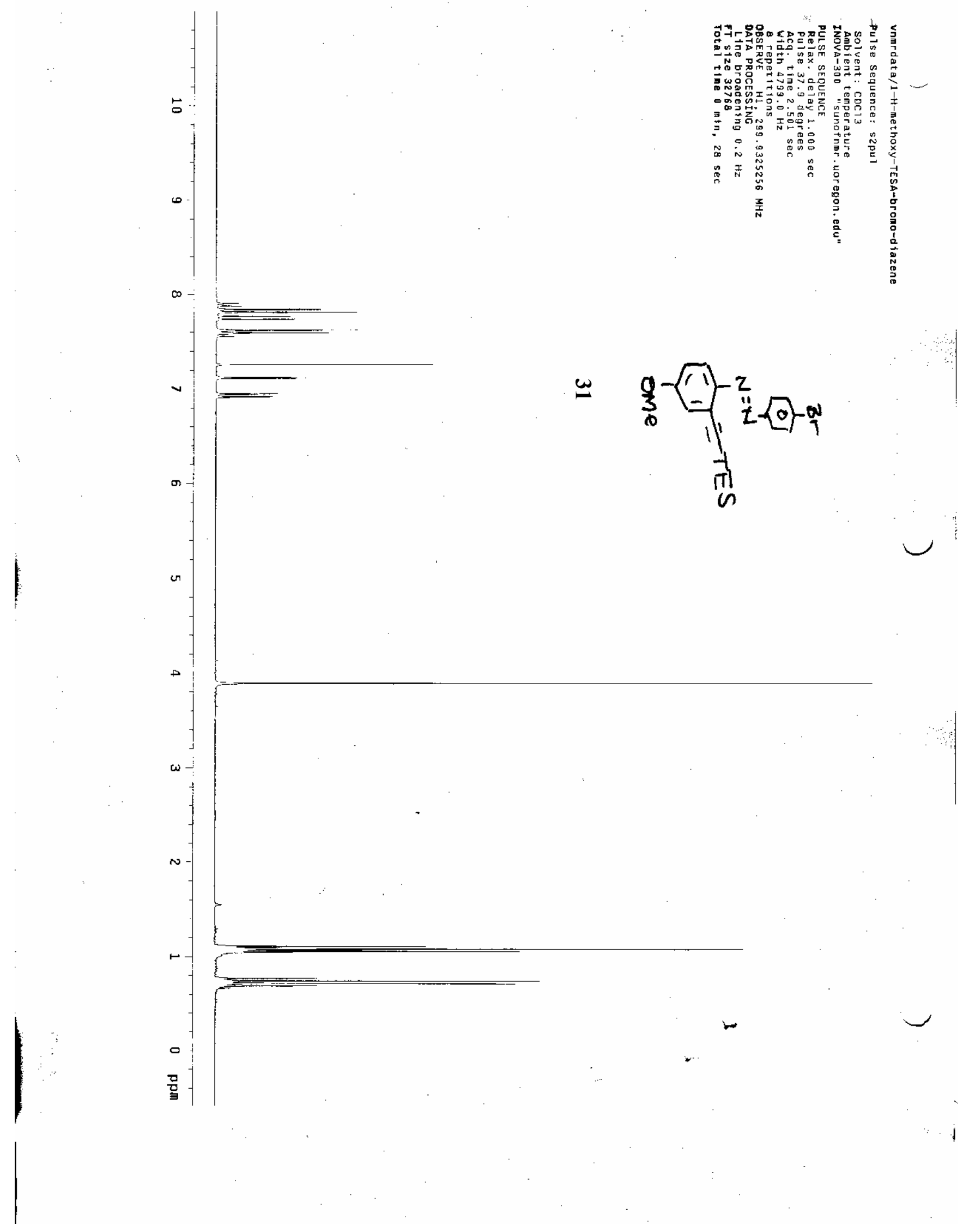



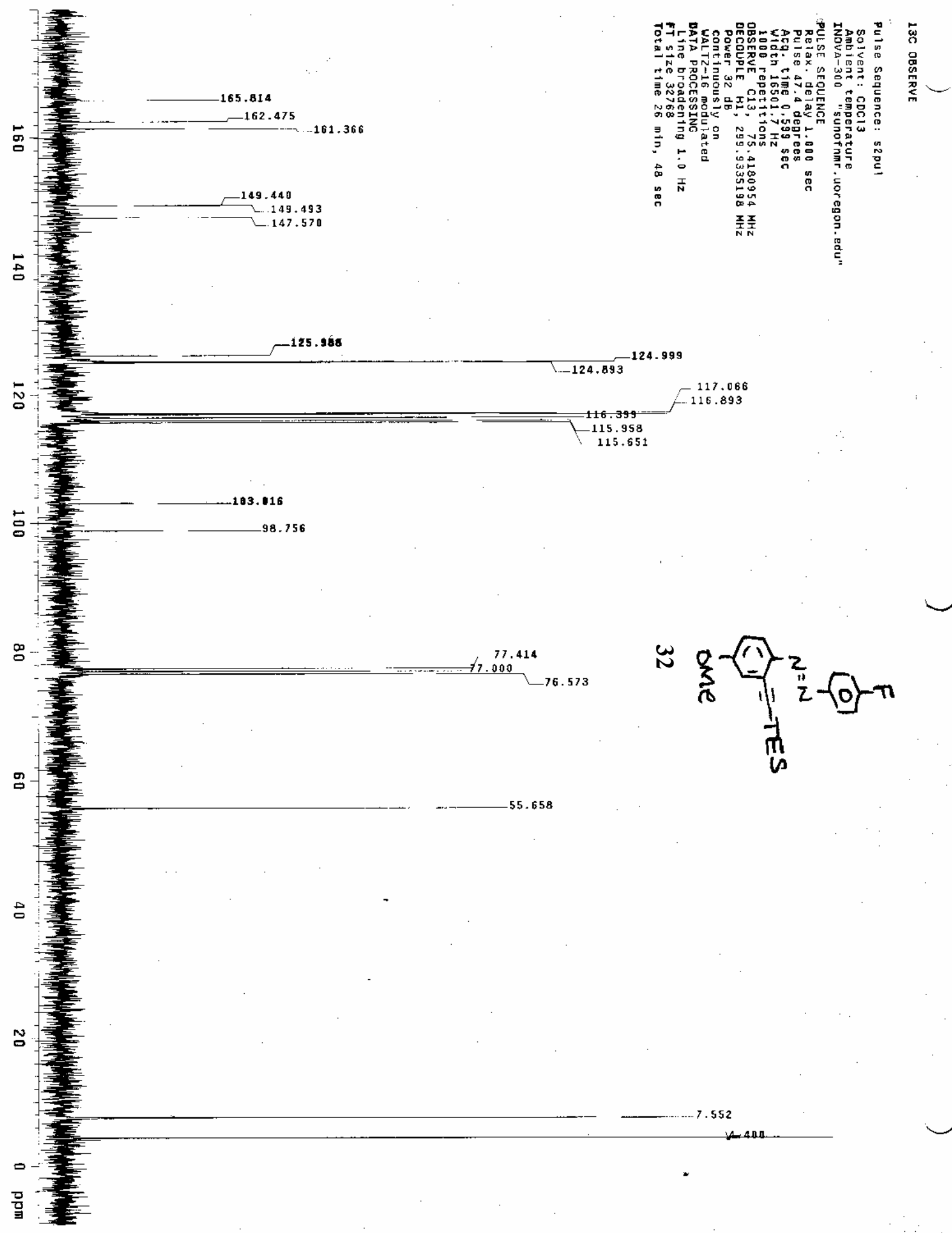


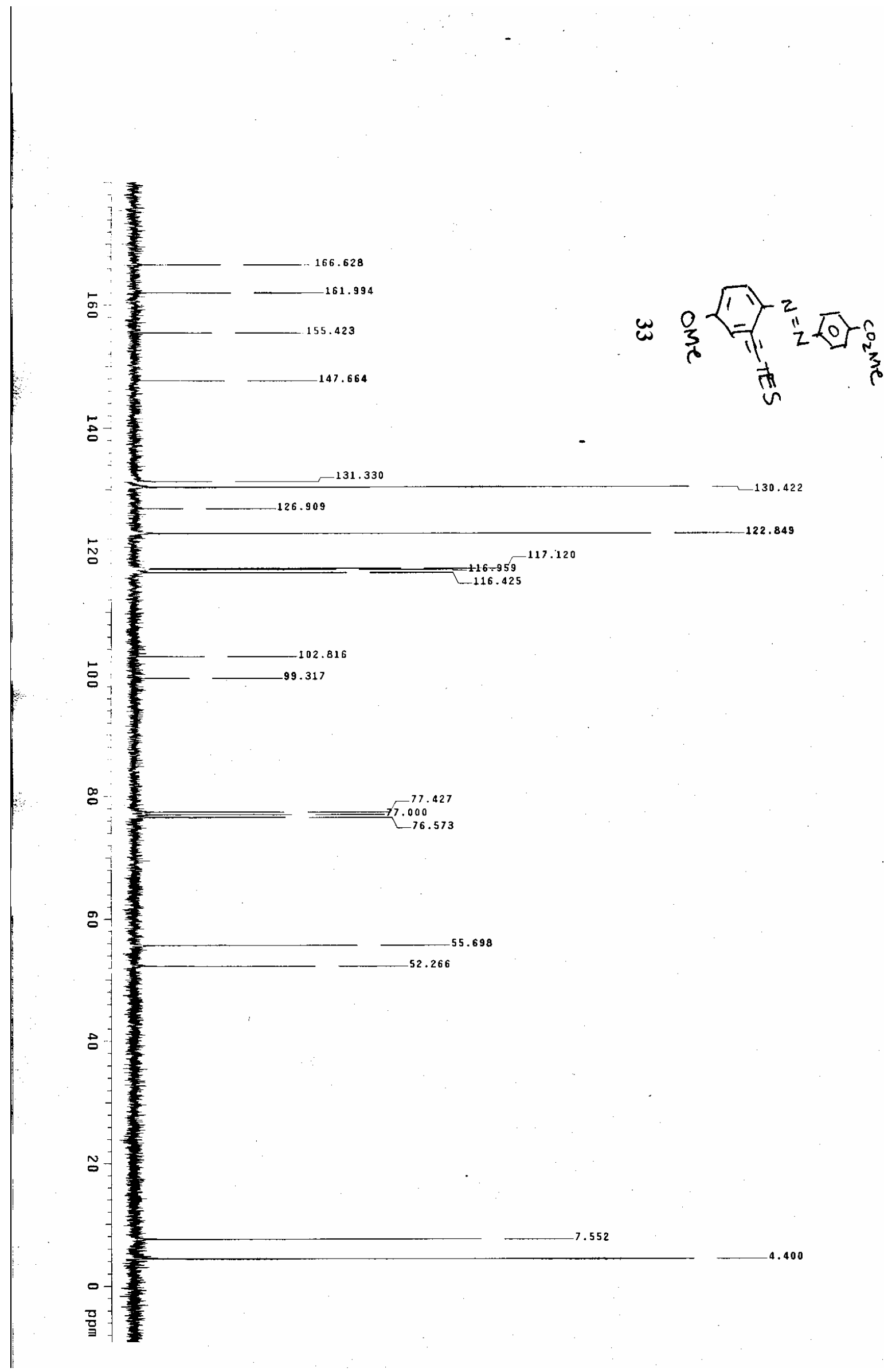

S 80 

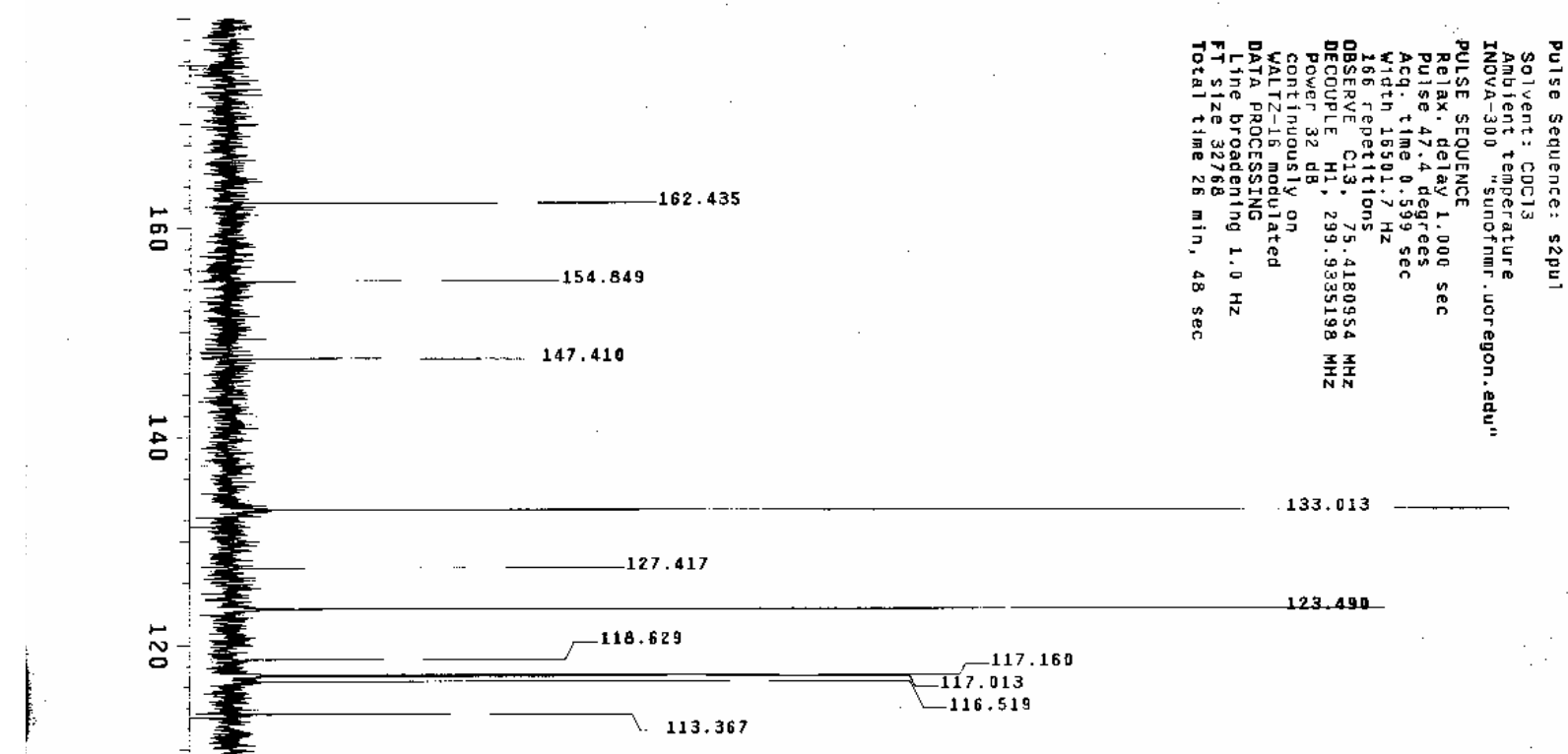

ㄴ.

$$
\text { - }
$$

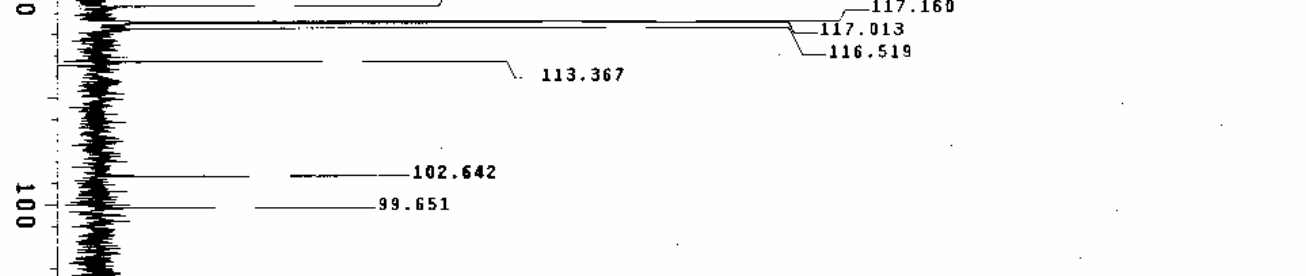

g

g

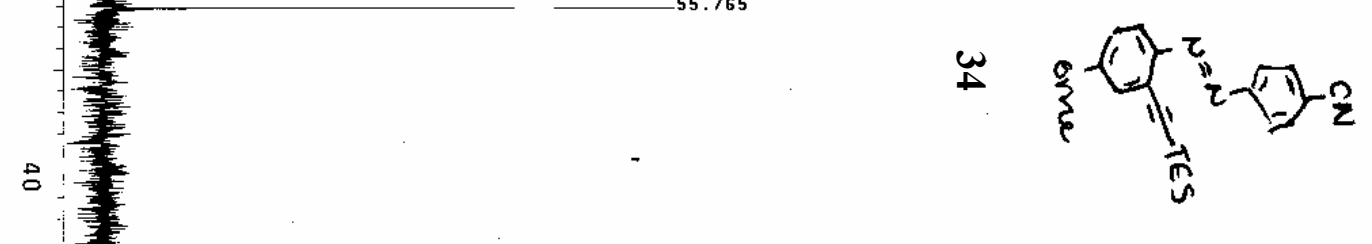

s

0

8
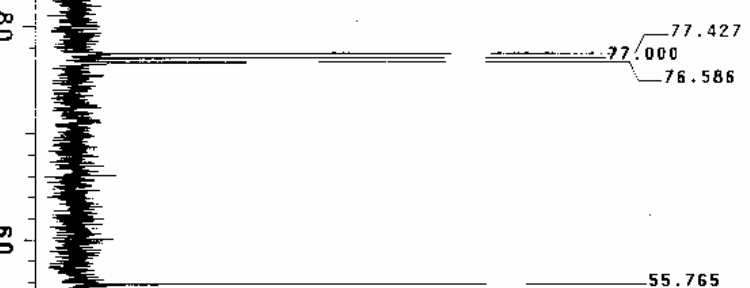

$-55.765$

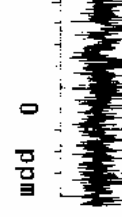

w323

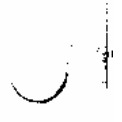



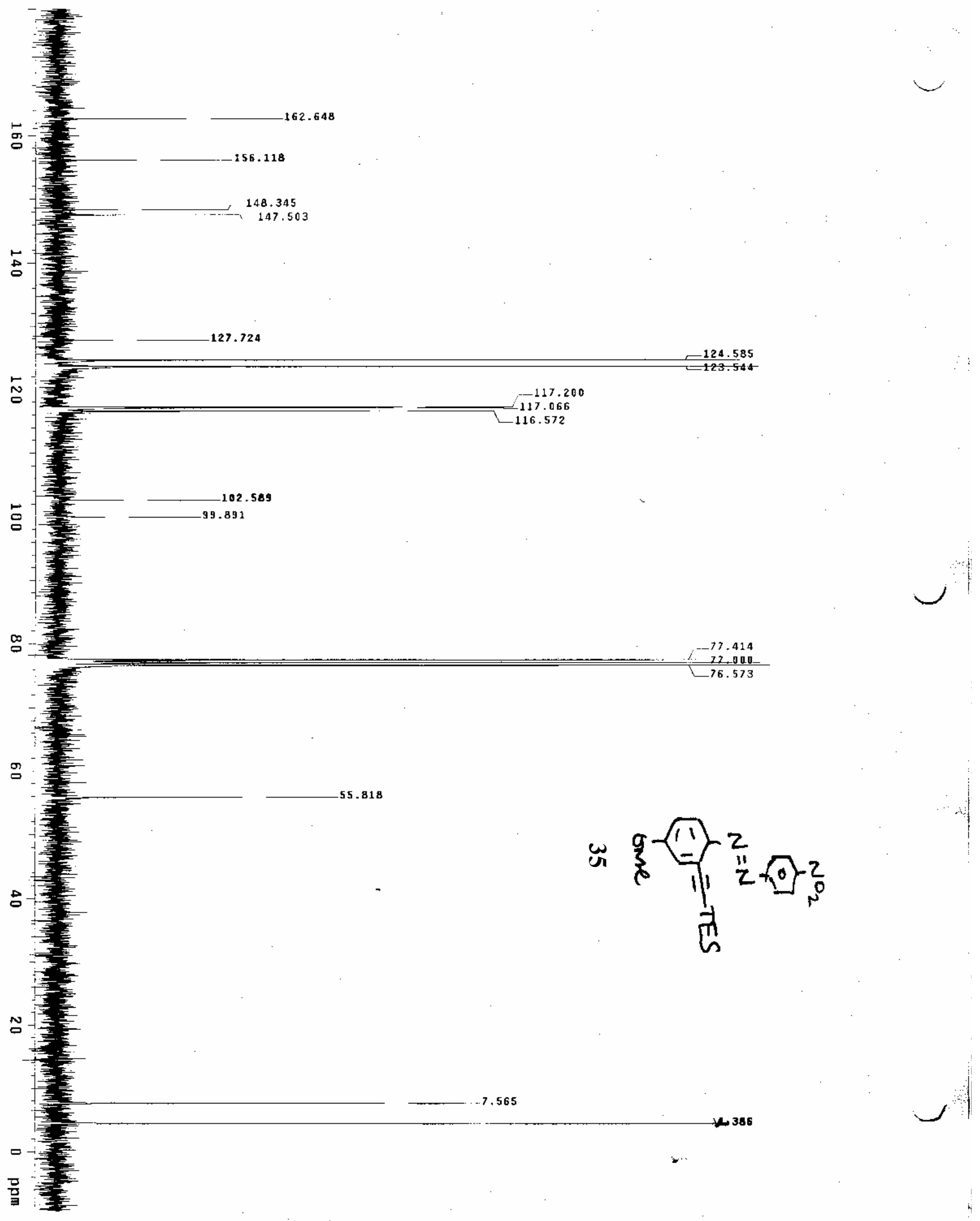


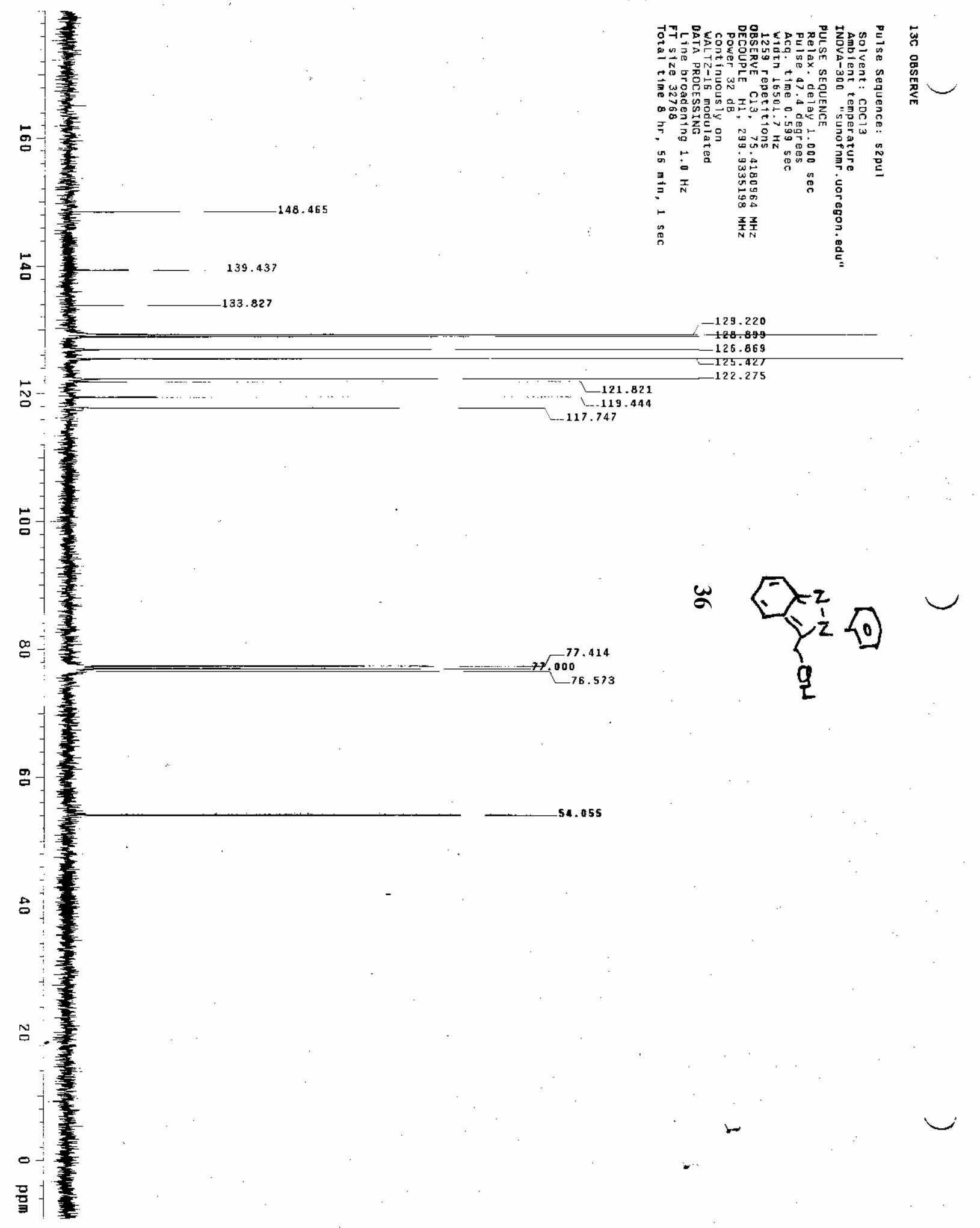




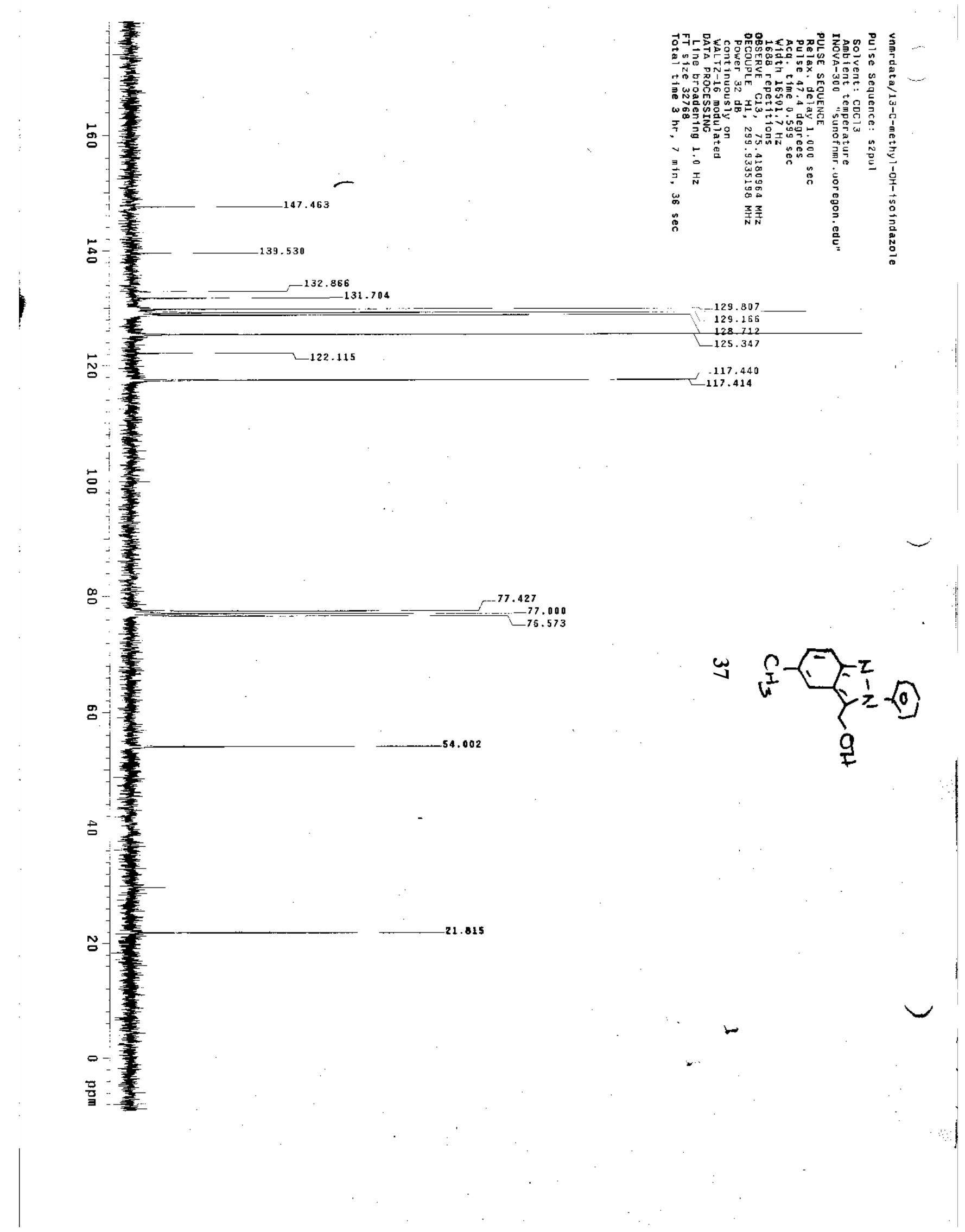




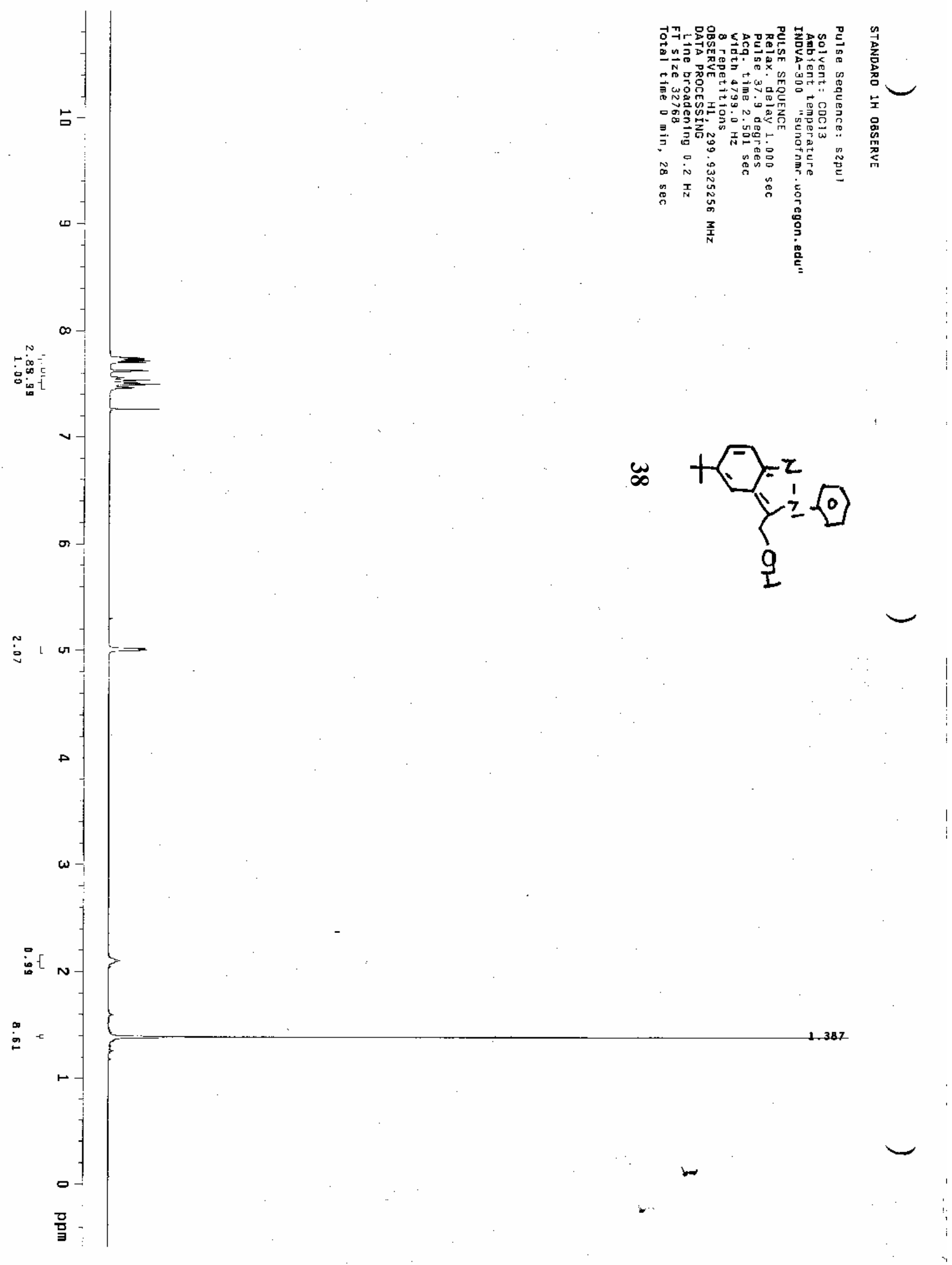




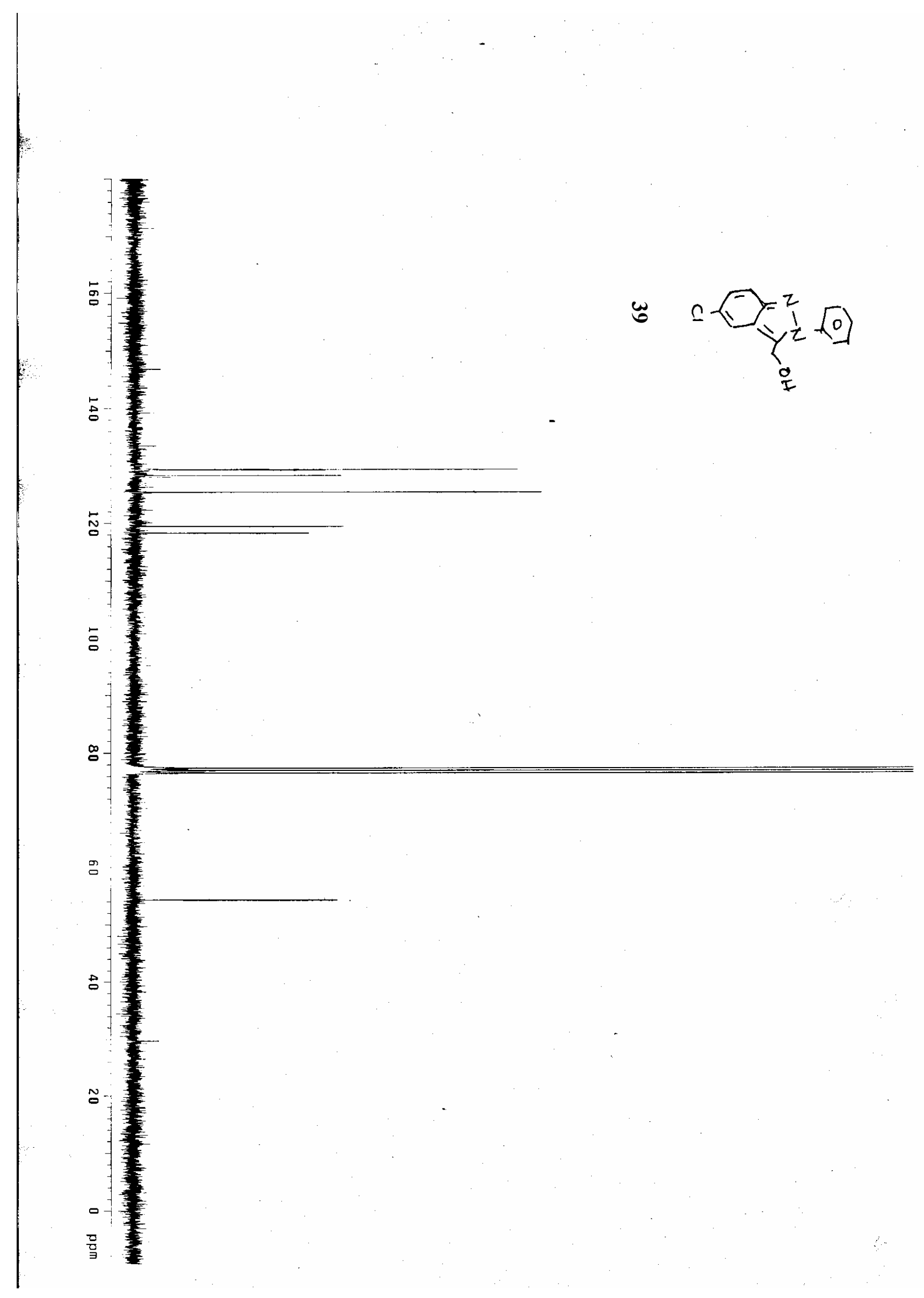




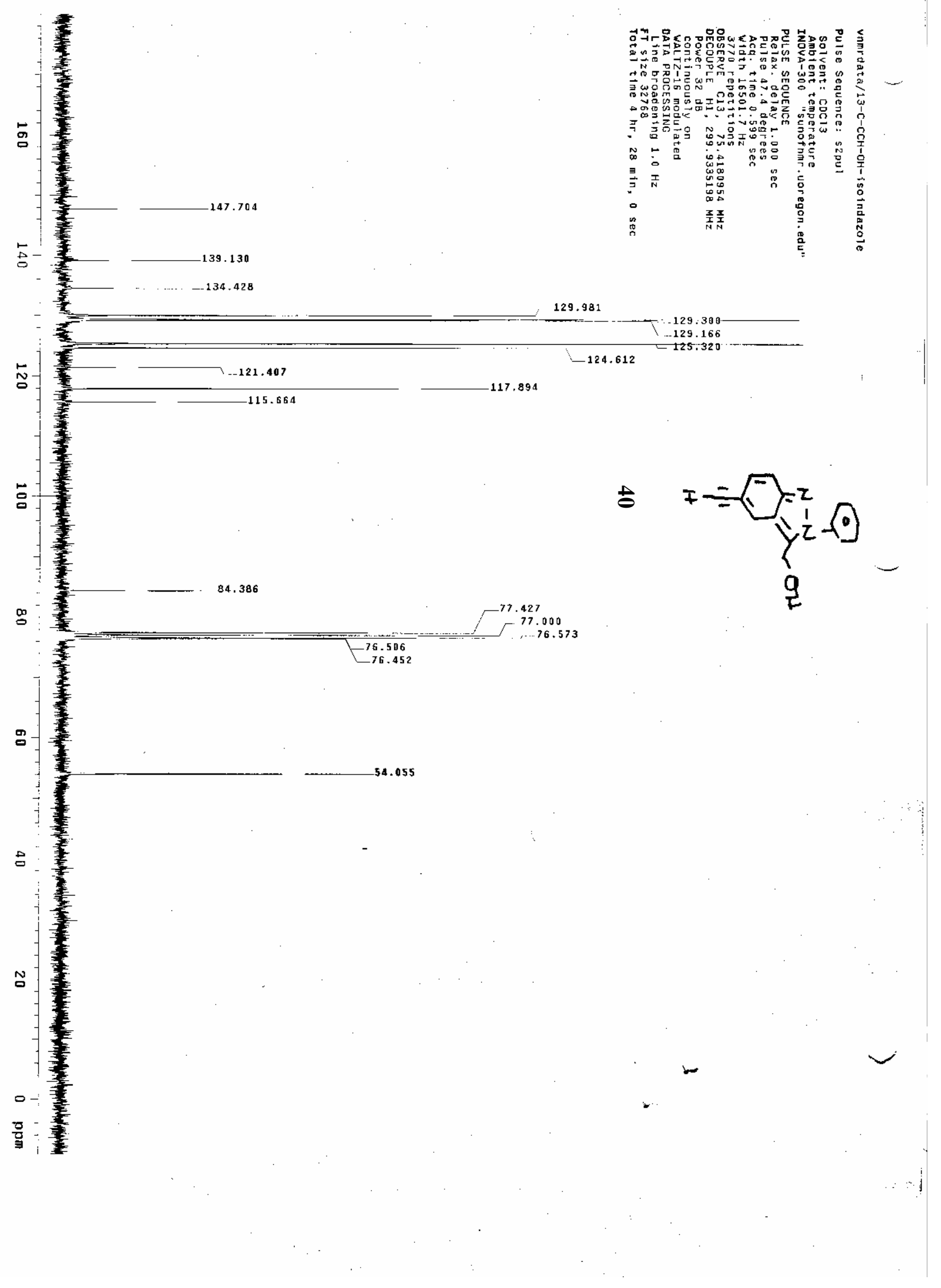




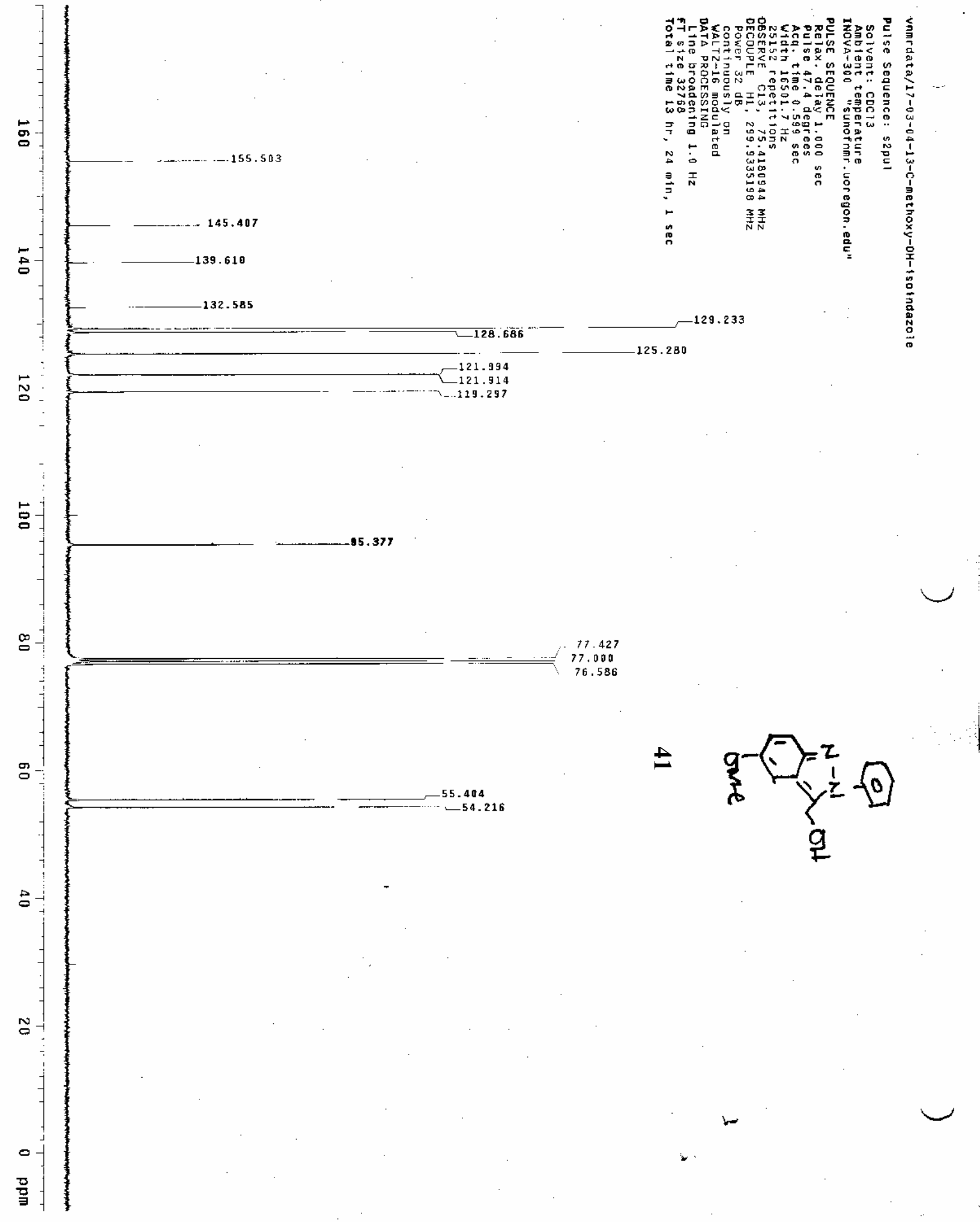




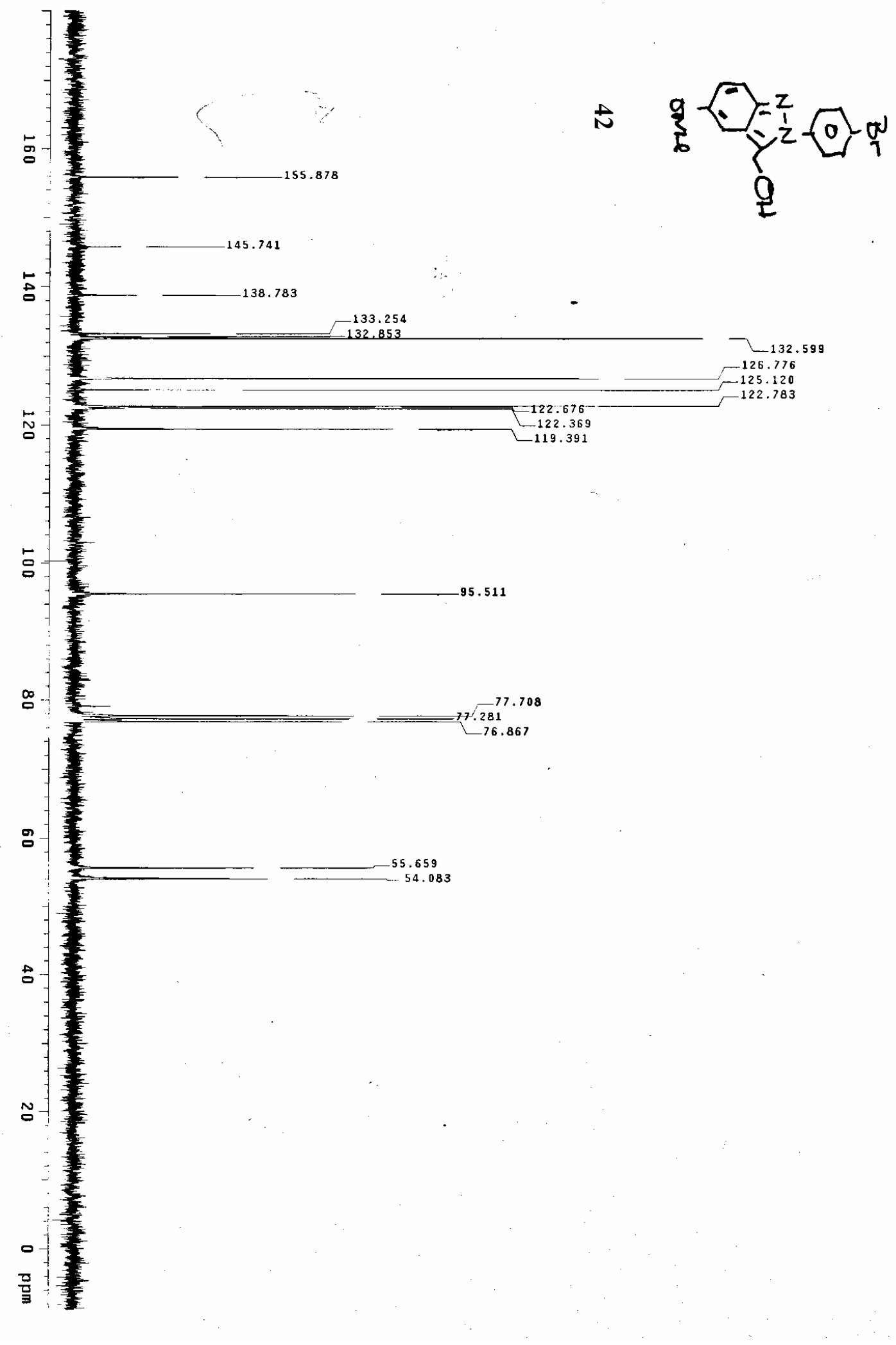




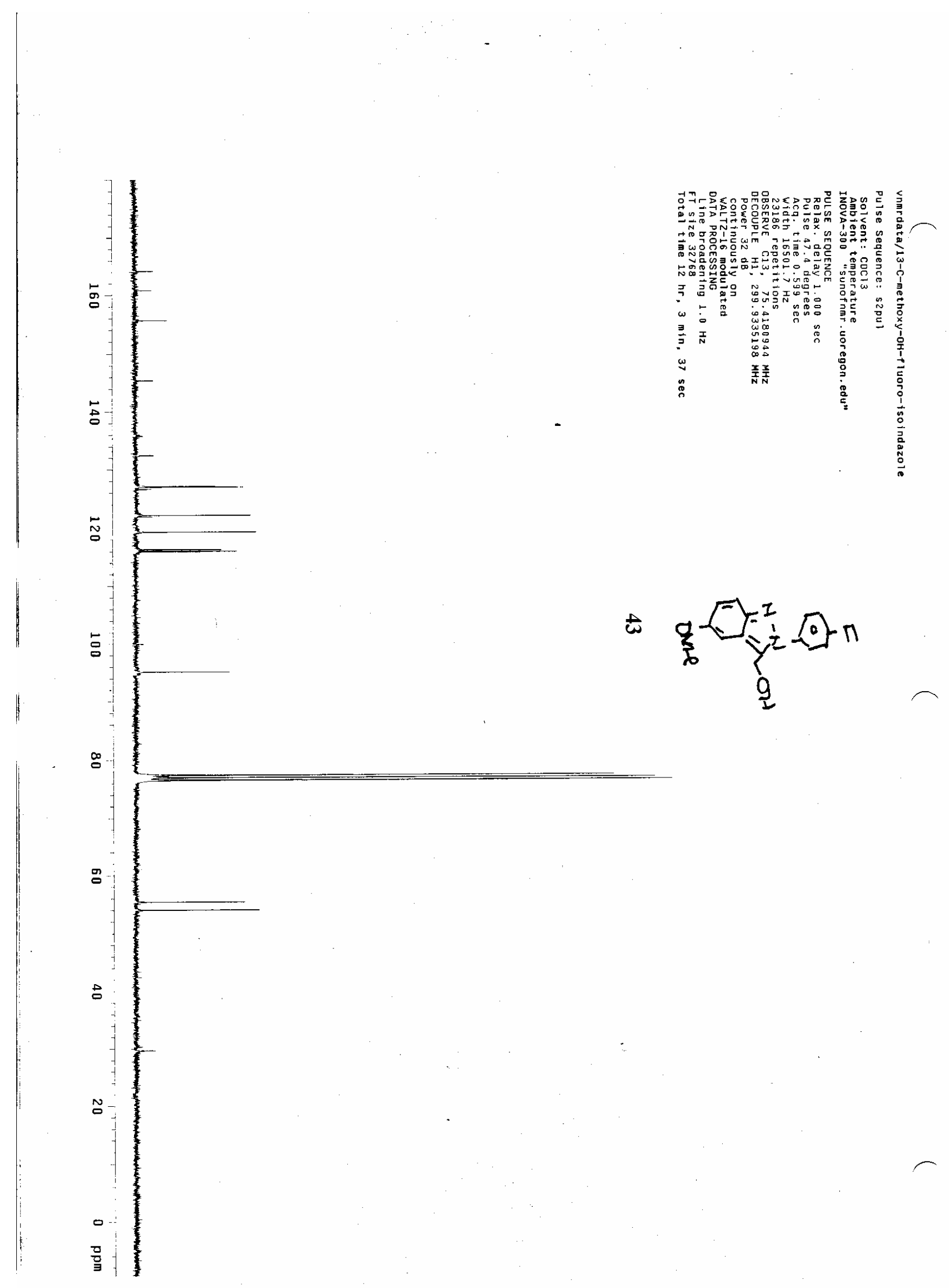




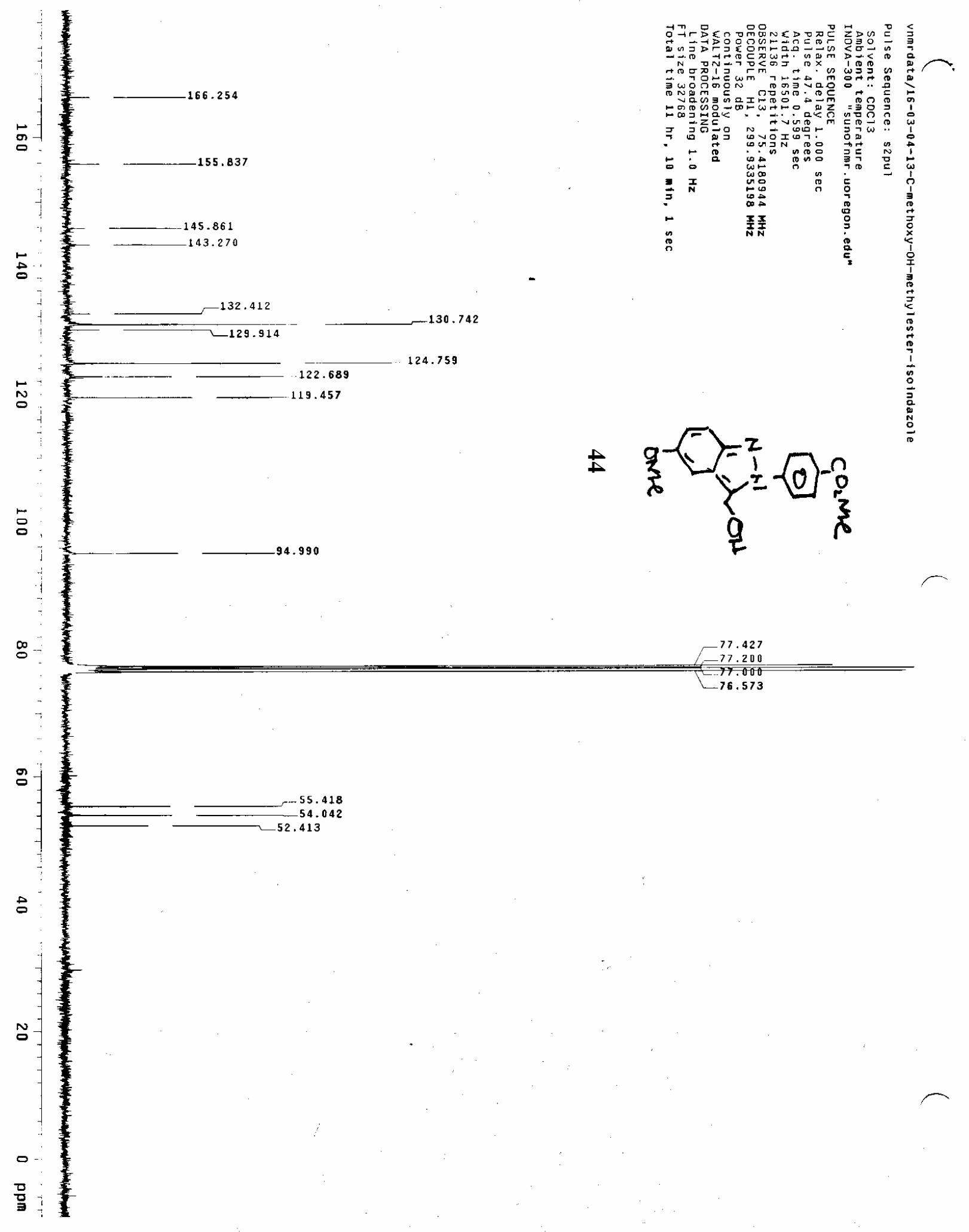




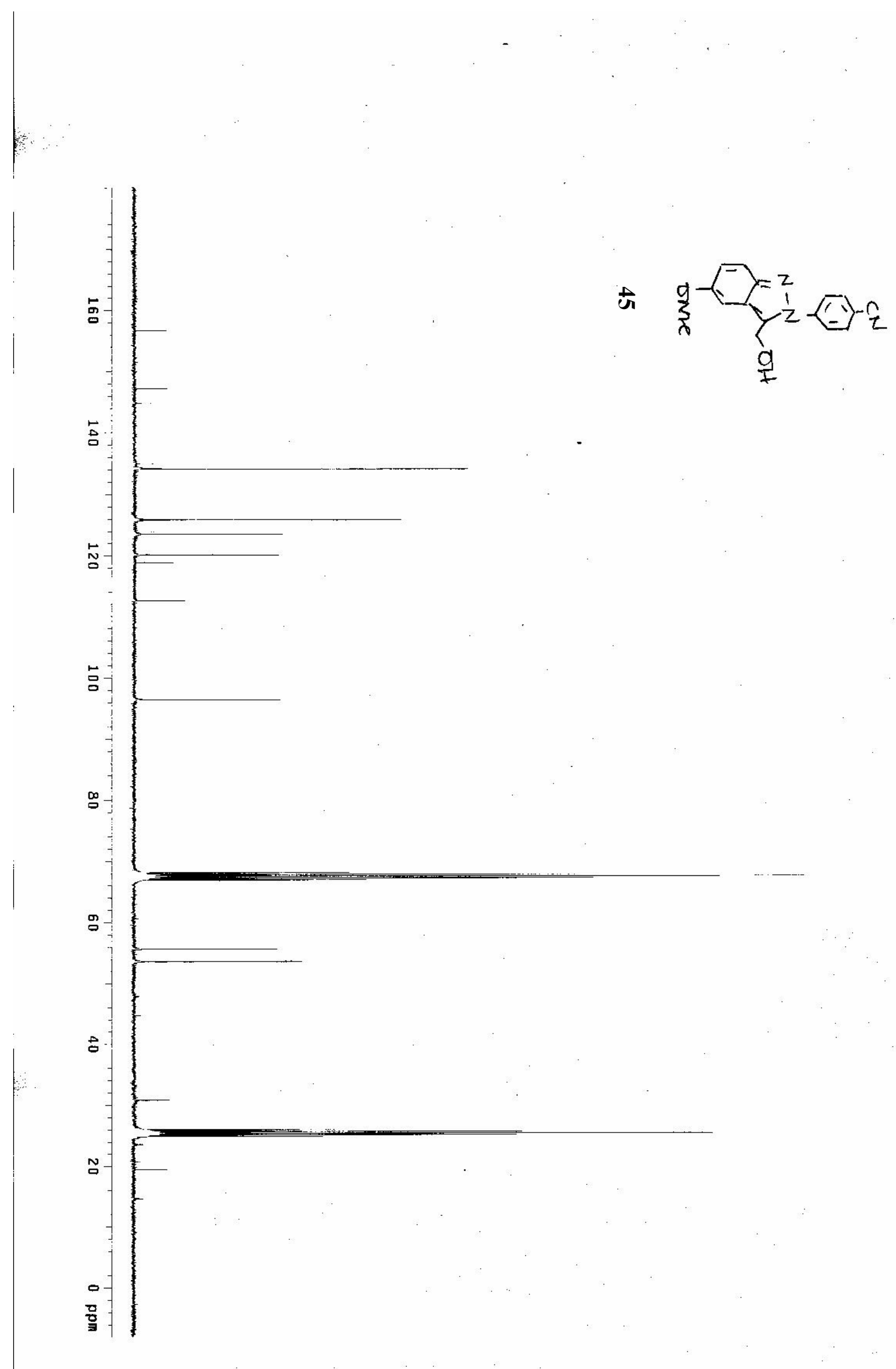




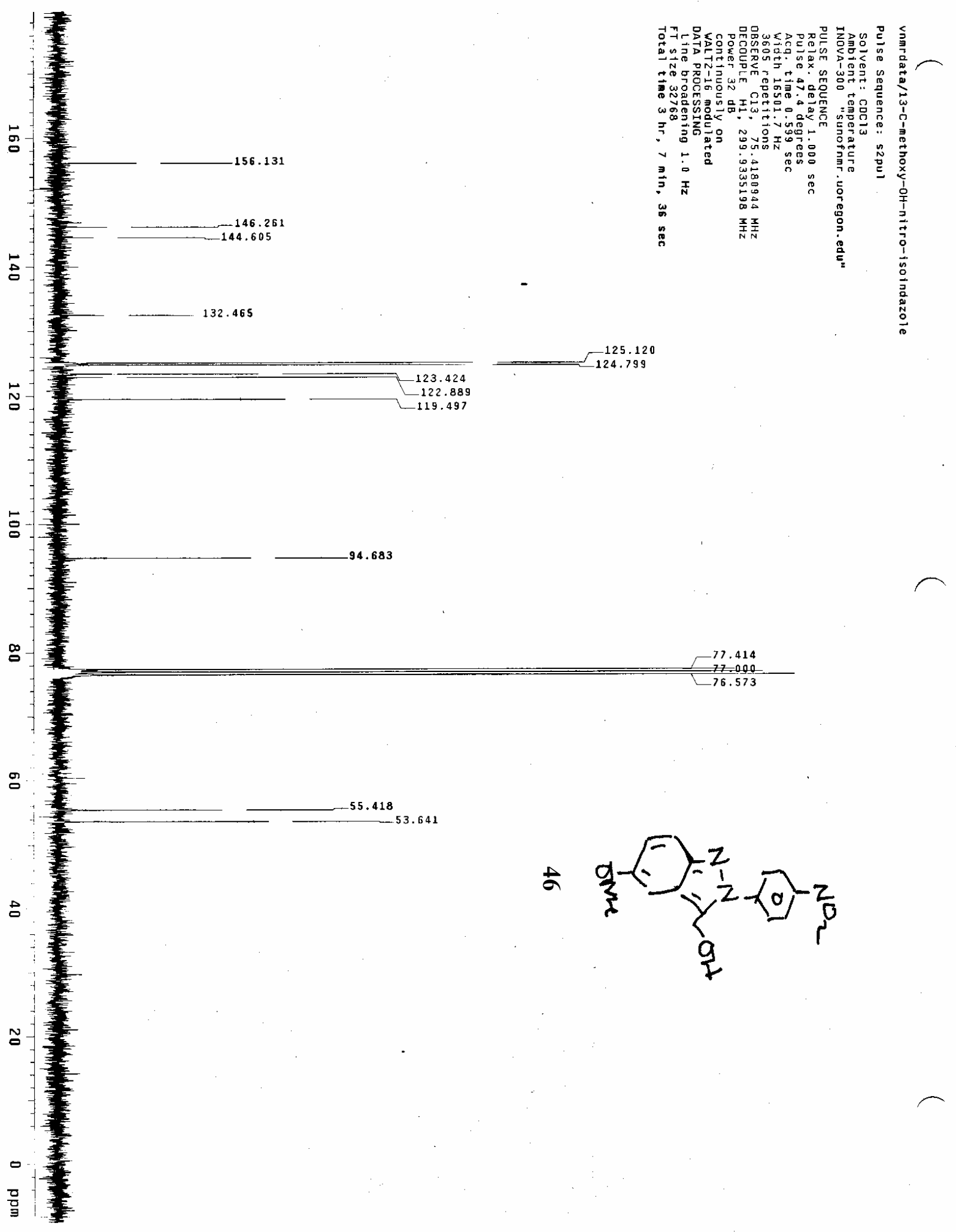




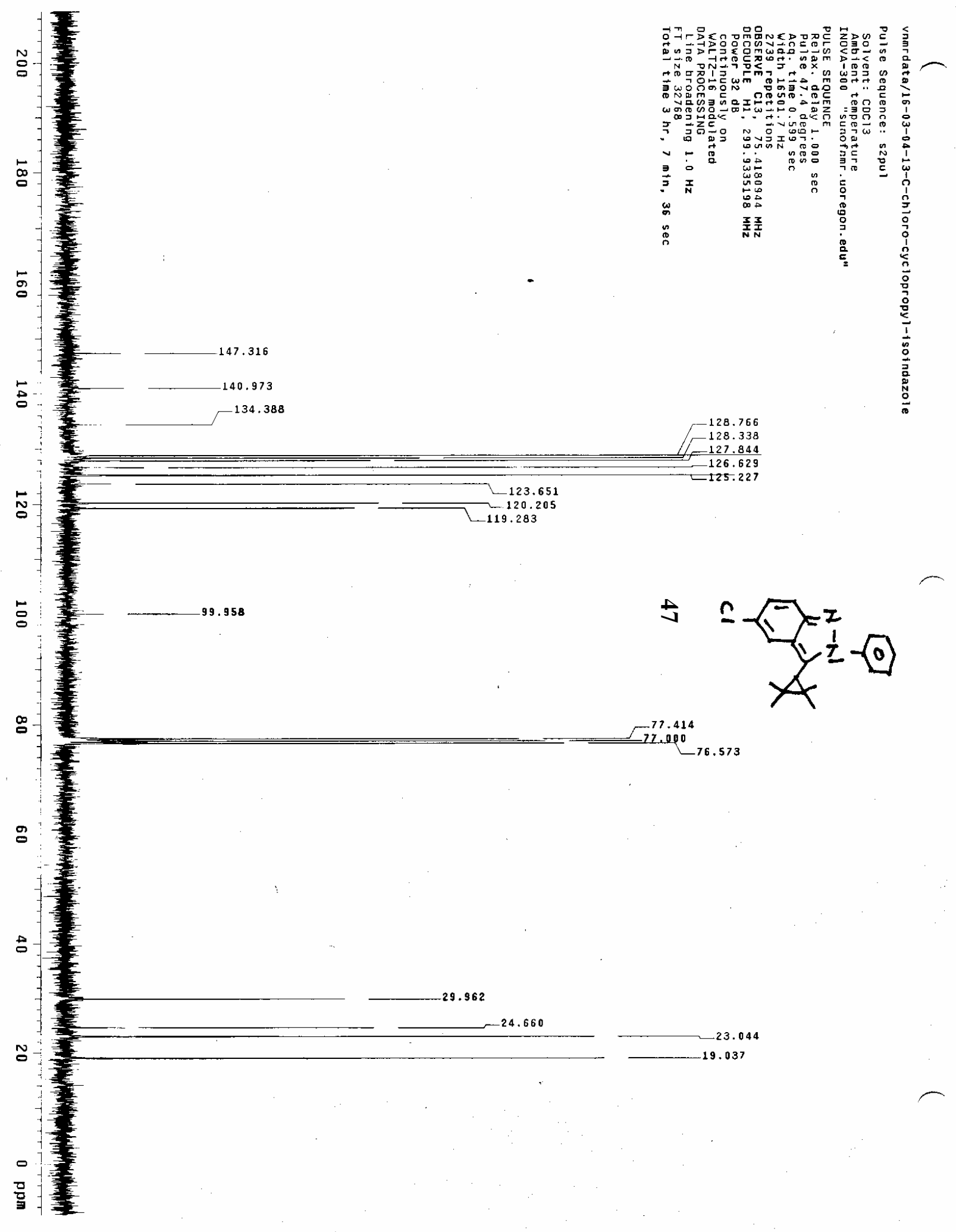

